\title{
THE MOTIVE OF THE HILBERT CUBE $X^{[3]}$
}

\author{
MINGMIN SHEN $^{1}$ and CHARLES VIAL ${ }^{2}$ \\ ${ }^{1}$ KdV Institute for Mathematics, University of Amsterdam, P.O. Box 94248, \\ 1090 GE Amsterdam, Netherlands; \\ email: m.shen@uva.nl \\ 2 DPMMS, University of Cambridge, Wilberforce Road, Cambridge CB3 0WB, UK; \\ email: c.vial@dpmms.cam.ac.uk
}

Received 10 April 2015; accepted 3 October 2016

\begin{abstract}
The Hilbert scheme $X^{[3]}$ of length-3 subschemes of a smooth projective variety $X$ is known to be smooth and projective. We investigate whether the property of having a multiplicative ChowKünneth decomposition is stable under taking the Hilbert cube. This is achieved by considering an explicit resolution of the rational map $X^{3} \rightarrow X^{[3]}$. The case of the Hilbert square was taken care of in Shen and Vial [Mem. Amer. Math. Soc. 240(1139) (2016), vii+163 pp]. The archetypical examples of varieties endowed with a multiplicative Chow-Künneth decomposition is given by abelian varieties. Recent work seems to suggest that hyperKähler varieties share the same property. Roughly, if a smooth projective variety $X$ has a multiplicative Chow-Künneth decomposition, then the Chow rings of its powers $X^{n}$ have a filtration, which is the expected Bloch-Beilinson filtration, that is split.
\end{abstract}

2010 Mathematics Subject Classification: 14C05 (primary); 14C25, 14C15 (secondary)

\section{Introduction}

Let $X$ be a smooth projective variety of dimension $d$, over a field $k$, which we assume is endowed with a Chow-Künneth decomposition $\left\{\pi_{X}^{i} \in \mathrm{CH}^{d}(X \times X)\right.$ : $0 \leqslant i \leqslant 2 d\}$. Informally, this means that the Künneth decomposition of the $\ell$-adic class $(\ell \neq$ char $k)$ of the diagonal $\left[\Delta_{X}\right] \in \mathrm{H}^{2 d}\left(X_{\bar{k}}, \mathbb{Q}_{\ell}\right)$ is algebraic and lifts to a splitting of the rational Chow motive of $X$. Precisely, there exist cycles $\pi_{X}^{i} \in$ $\mathrm{CH}^{d}(X \times X)$ with rational coefficients such that $\Delta_{X}=\sum_{i=0}^{2 d} \pi_{X}^{i}$ and which satisfy, when seen as self-correspondences of $X, \pi_{X}^{i} \circ \pi_{X}^{i}=\pi_{X}^{i}, \pi_{X}^{i} \circ \pi_{X}^{j}=0$ for $i \neq j$,

(C) The Author(s) 2016. This is an Open Access article, distributed under the terms of the Creative Commons Attribution licence (http://creativecommons.org/licenses/by/4.0/), which permits unrestricted re-use, distribution, and reproduction in any medium, provided the original work is properly cited. 
and $\left(\pi_{X}^{i}\right)_{*} \mathrm{H}^{*}\left(X_{\bar{k}}, \mathbb{Q}_{\ell}\right)=\mathrm{H}^{i}\left(X_{\bar{k}}, \mathbb{Q}_{\ell}\right)$. Such a decomposition induces a descending filtration on the Chow groups of $X$ by the formula

$$
\mathrm{F}^{s} \mathrm{CH}^{i}(X):=\left(\sum_{l \leqslant 2 i-s} \pi_{X}^{l}\right)_{*} \mathrm{CH}^{i}(X) .
$$

The conjectures of Murre [10] predict that

(B) $\mathrm{CH}^{i}(X)=\mathrm{F}^{0} \mathrm{CH}^{i}(X)$ and $\mathrm{F}^{s} \mathrm{CH}^{i}(X)=0$ when $s>i$;

(D) $\mathrm{F}^{1} \mathrm{CH}^{i}(X)=\operatorname{Ker}\left\{c l: \mathrm{CH}^{i}(X) \rightarrow \mathrm{H}^{2 i}\left(X_{\bar{k}}, \mathbb{Q}_{\ell}\right)\right\}$;

(C) any two Chow-Künneth decompositions for $X$ induce the same filtration $\mathrm{F}^{\bullet}$ on $\mathrm{CH}^{i}(X)$.

By Jannsen (see [7] for precise statements), the filtration induced by a ChowKünneth decomposition should be the filtration conjectured by Bloch and Beilinson and, conversely, the filtration conjectured by Bloch and Beilinson should be induced by any Chow-Künneth decomposition. In particular, this filtration should actually be a filtration on the Chow ring $\mathrm{CH}^{*}(X)$ of $X$. Because of Jannsen's result, we will refer to a filtration on the Chow groups of $X$ induced by a Chow-Künneth decomposition as a filtration of expected Bloch-Beilinson type.

Every smooth projective variety is conjectured by Murre [10] to be endowed with a Chow-Künneth decomposition. Examples of varieties for which the existence of a Chow-Künneth decomposition has been settled include curves, surfaces [9] and abelian varieties [1]. A natural question is can one show that, provided a Chow-Künneth decomposition for $X$, the induced filtration (which is expected to be the Bloch-Beilinson filtration) is a filtration on the Chow ring of $X$ ? That is can one show that the induced filtration on the Chow groups of $X$ is compatible with intersection product? Before moving on to a more specific question, let us mention that the answer in the cases listed above (curves, surfaces and abelian varieties) is yes.

While it is expected that the Bloch-Beilinson filtration, when it exists, is a filtration on the Chow ring of $X$, one cannot expect this filtration on the Chow ring of $X$ to be split in general. Indeed, by the expected properties of the Bloch-Beilinson filtration, one expects the graded pieces $\operatorname{Gr}_{\mathrm{F}}^{0} \mathrm{CH}^{i}(X)$ to inject into $\mathrm{H}^{2 i}\left(X_{\bar{k}}, \mathbb{Q}_{\ell}\right)$ under the cycle class map. In particular $\operatorname{Gr}_{\mathrm{F}}^{0} \mathrm{CH}^{d}(X)$ is one-dimensional, where $d=\operatorname{dim} X$. On the other hand, one also expects that $\mathrm{F}^{0} \mathrm{CH}^{1}(X)=\mathrm{CH}^{1}(X)$ and $\mathrm{F}^{1} \mathrm{CH}^{1}(X)=0$ when $\mathrm{H}^{1}\left(X_{\bar{k}}, \mathbb{Q}_{\ell}\right)=0$. Therefore, if $X$ is such that $\mathrm{H}^{1}\left(X_{\bar{k}}, \mathbb{Q}_{\ell}\right)=0$ and if the conjectural Bloch-Beilinson filtration is split, then the image of the intersection product map $\mathrm{CH}^{1}(X) \otimes \cdots \otimes$ 
$\mathrm{CH}^{1}(X) \rightarrow \mathrm{CH}^{d}(X)$ is one-dimensional. There are however some examples of simply connected surfaces $X$ for which the rank of the image of the intersection product $\mathrm{CH}^{1}(X) \otimes \mathrm{CH}^{1}(X) \rightarrow \mathrm{CH}^{2}(X)$ is $\geqslant 2$. (Consider for instance the blowup of a simply connected surface $S$ with $\operatorname{dim} \mathrm{CH}_{0}(S) \geqslant 2$ at a point that is not rationally equivalent to any cycle in $\operatorname{Im}\left\{\mathrm{CH}^{1}(S) \otimes \mathrm{CH}^{1}(S) \rightarrow \mathrm{CH}^{2}(S)\right\}$; then the self-intersection of the exceptional divisor provides a new dimension which was not in the image of the intersection product.) There are nonetheless examples of varieties for which the filtration splits. For example, Beauville [1] proved that the filtration splits for abelian varieties. Ten years ago, Beauville and Voisin [3] observed that the filtration also splits for K3 surfaces. Then Beauville [2], after studying intersection of divisors on certain hyperKähler varieties, asked whether the conjectural Bloch-Beilinson filtration, if it exists, would split for all hyperKähler varieties. Here, a hyperKähler variety refers to a projective irreducible holomorphic symplectic manifold. This question was answered affirmatively in [12] in the case when $X$ is the Hilbert scheme of length2 subschemes on a K3 surface or the variety of lines on a generic cubic 4-fold, and in [14] in the case when $X$ is the Hilbert scheme of length- $n$ subschemes on a K3 surface for any integer $n$.

In fact, when $X$ is the Hilbert scheme $S^{[n]}$ of length- $n$ subschemes on a K3 surface $S$, we established in $[12,14]$ a stronger statement. In order to motivate that statement, let us make the following observation. Recall that if $X$ and $Y$ are smooth projective varieties with a Chow-Künneth decomposition, then the product $X \times Y$ is naturally endowed with the product Chow-Künneth decomposition $\pi_{X \times Y}^{k}:=\sum_{i+j=k} \pi_{X}^{i} \otimes \pi_{Y}^{j}$ (see (4) for the definition of the tensor product of correspondences seen as morphisms of Chow motives). However, having a Chow-Künneth decomposition inducing a filtration on the Chow ring that is split is not stable under product. Indeed, any Chow-Künneth decomposition on a curve induces a filtration that is split (there is nothing to intersect on a curve) but in general the filtration induced on the product of two curves is not split. A nicer notion, that is stable under product, is that of multiplicative Chow-Künneth decomposition. A Chow-Künneth decomposition $\left\{\pi_{X}^{i}: 0 \leqslant i \leqslant 2 d\right\}$ is said to be multiplicative if

$$
\pi_{X}^{k} \circ \Delta_{123} \circ\left(\pi_{X}^{i} \otimes \pi_{X}^{j}\right)=0 \in \mathrm{CH}^{2 d}(X \times X \times X) \quad \text { whenever } k \neq i+j .
$$

Here, $\Delta_{123} \in \mathrm{CH}^{2 d}(X \times X \times X)$ is the class of the small diagonal $\{(x, x, x): x \in$ $X$ \} seen as a correspondence from $X \times X$ to $X$. At this point it should be noted that the relations (1) always hold modulo homological equivalence, and that if $\alpha$ and $\beta$ are cycles in $\mathrm{CH}^{*}(X)$, then $\left(\Delta_{123}\right)_{*}(\alpha \times \beta)=\alpha \cdot \beta$. If $X$ admits a multiplicative Chow-Künneth decomposition, then the induced filtration on the Chow groups of $X$ is a filtration on the Chow ring of $X$ that is split. Indeed, given a Chow- 
Künneth decomposition $\left\{\pi_{X}^{i}\right\}$ for $X$ and writing (whether $\left\{\pi_{X}^{i}\right\}$ is multiplicative or not)

$$
\mathrm{CH}^{i}(X)_{s}:=\left(\pi_{X}^{2 i-s}\right)_{*} \mathrm{CH}^{i}(X),
$$

the condition that $\left\{\pi_{X}^{i}\right\}$ is multiplicative implies that $\mathrm{CH}^{*}(X)=\bigoplus_{i, s} \mathrm{CH}^{i}(X)_{s}$ is a bigraded ring, that is, $\mathrm{CH}^{i}(X)_{s} \cdot \mathrm{CH}^{j}(X)_{t} \subseteq \mathrm{CH}^{i+j}(X)_{s+t}$. As a matter of fact (see [12, Section 8]), K3 surfaces and abelian varieties not only have a filtration of expected Bloch-Beilinson type that is split but also have a multiplicative Chow-Künneth decomposition by work of Beauville-Voisin [3] and Beauville [1], respectively. In [12], we proved, under the technical, but yet natural, assumption that the Chern classes $c_{p}(X)$ belong to the graded-0 part $\mathrm{CH}^{p}(X)_{0}$ of $\mathrm{CH}^{p}(X)$, that if $X$ is a smooth projective variety that admits a multiplicative Chow-Künneth decomposition (for example $X$ a K3 surface), then the Hilbert scheme of length-2 subschemes $X^{[2]}$ also admits a multiplicative Chow-Künneth decomposition.

Given a smooth projective variety $X$, Cheah [4] showed that the Hilbert scheme $X^{[3]}$ of length-3 subschemes of $X$ is smooth and projective. (Cheah also showed that if $\operatorname{dim} X \geqslant 3$, the Hilbert scheme $X^{[n]}$ is never smooth when $n \geqslant 4$.) In this manuscript, we want to push further the method of [12, Section 13] to show that whenever $X$ admits a multiplicative Chow-Künneth decomposition, then its Hilbert cube $X^{[3]}$ also admits a multiplicative Chow-Künneth decomposition. The idea is basic: we resolve the rational map $X^{3}-\rightarrow X^{[3]}$ by successively blowing up subvarieties that are invariant under the action of the symmetric group $\mathfrak{S}_{3}$. We obtain a generically finite morphism denoted $p: X_{3} \rightarrow X^{[3]}$, and we check that the properties of the Chow-Künneth decomposition of $X^{3}$ induced by that of $X$ (self-duality, multiplicativity, Chern classes belonging to the graded-0 part of the Chow groups) lift to $X_{3}$. We then check, and this requires a careful analysis of the geometry of $X^{[3]}$, that the resulting multiplicative Chow-Künneth decomposition on $X_{3}$ descends along the morphism $p$. The main result of this paper is the following.

THEOREM 1. Let $X$ be a smooth projective variety that admits a self-dual multiplicative Chow-Künneth decomposition (see Definitions 2.1 and 3.1). Assume that the Chern classes of $X$ satisfy $c_{p}(X) \in \mathrm{CH}^{p}(X)_{0}$. Then the Hilbert cube $X^{[3]}$ also admits a self-dual multiplicative Chow-Künneth decomposition, with the property that the Chern classes $c_{p}\left(X^{[3]}\right)$ sit in $\mathrm{CH}^{p}\left(X^{[3]}\right)_{0}$.

In particular, still assuming $c_{p}(X) \in \mathrm{CH}^{p}(X)_{0}$, the Chow ring $\mathrm{CH}^{*}\left(X^{[3]}\right)$ has a filtration, which is the candidate Bloch-Beilinson filtration, that is split. 
Here, a self-dual Chow-Künneth decomposition means a Chow-Künneth decomposition $\left\{\pi_{X}^{i}, 0 \leqslant i \leqslant 2 d\right\}$ such that $\pi_{X}^{2 d-i}$ is the transpose of $\pi_{X}^{i}$ for all $i$. The self-duality assumption on $\left\{\pi_{X}^{i}\right\}$ is important because it ensures, together with the multiplicativity assumption, that the classes of the several diagonals in $X^{3}$ belong to $\mathrm{CH}^{*}\left(X^{3}\right)_{0}$, which is crucial for checking that the assumptions of Proposition 3.4 are met for $X^{3}$ and its diagonals. We also take the trouble of showing that a blow-up admits a self-dual Chow-Künneth decomposition (Proposition 2.10) essentially because we will have to blow up $X^{3}$ several times and at each step self-duality will be required. Ultimately we find that $X^{[3]}$ admits a multiplicative Chow-Künneth decomposition that is self-dual and this makes it possible to iterate the process, for example, to show that $\left(X^{[3]}\right)^{[3]}$ also has a multiplicative Chow-Künneth decomposition.

Beyond the case of Hilbert schemes of points, one may consider nested Hilbert schemes. Given a projective variety $X$ and positive integers $n_{1}<\cdots<n_{l}$, the nested Hilbert scheme $X^{\left[n_{1}, \ldots, n_{l}\right]}$ is the projective scheme consisting of $\left\{\left(x_{1}, \ldots, x_{l}\right): x_{1} \subset \cdots \subset x_{l}\right\} \subset X^{\left[n_{1}\right]} \times \cdots \times X^{\left[n_{l}\right]}$. Cheah [4] showed that for a smooth projective variety $X$ of dimension $\geqslant 3$, the nested Hilbert scheme $X^{\left[n_{1}, \ldots, n_{l}\right]}$ is smooth if and only if it is one of $X^{[1,2]}$ or $X^{[2,3]}$. In Theorem 7.1, we establish the analogue of Theorem 1 in those cases, by showing that $X^{[1,2]}$ or $X^{[2,3]}$ admit a selfdual multiplicative Chow-Künneth decomposition with Chern classes belonging to the graded- 0 part of the Chow ring, whenever $X$ has a self-dual multiplicative Chow-Künneth decomposition with $c_{p}(X) \in \mathrm{CH}^{p}(X)_{0}$ for all $p \geqslant 0$.

Together with [12, Theorem 6], [14, Theorem 1] and [5, Theorem 1.7], we then obtain a large class of varieties admitting a multiplicative Chow-Künneth decomposition:

THEOREM 2. Let $E$ be the smallest subset of smooth projective varieties that contains varieties with Chow groups of finite rank (as $\mathbb{Q}$-vector spaces), abelian varieties, generalized Kummer varieties, and Hilbert schemes of length- $n$ subschemes of hyperelliptic curves, K3 surfaces, or abelian surfaces, and that is stable under the following operations:

(i) if $X$ and $Y$ belong to $E$, then $X \times Y \in E$;

(ii) if $X$ belongs to $E$, then $\mathbb{P}\left(\mathscr{T}_{X}\right) \in E$, where $\mathscr{T}_{X}$ is the tangent bundle of $X$;

(iii) if $X$ belongs to $E$, then the Hilbert scheme of length-2 subschemes $X^{[2]}$ belongs to $E$;

(iv) if $X$ belongs to $E$, then the Hilbert scheme of length-3 subschemes $X^{[3]}$ belongs to $E$; 
(v) if $X$ belongs to $E$, then the nested Hilbert schemes $X^{[1,2]}$ and $X^{[2,3]}$ belong to E.

If $X$ be a smooth projective variety that is isomorphic to a variety in $E$, then $X$ admits a self-dual multiplicative Chow-Künneth decomposition.

When the ground field $k$ has characteristic zero, Riess [11] showed that birational hyperKähler varieties have isomorphic Chow rings and isomorphic Chow motives as algebras in the category of Chow motives. Therefore, one may add to the set $E$ all hyperKähler varieties that are birational to the Hilbert scheme of length- $n$ subschemes on a K3 surface. In fact, by the following conjecture which is directly inspired from Beauville's splitting conjecture [2] and which we stated in $[12,14]$, we expect the set $E$ of Theorem 2 to contain all hyperKähler varieties.

CONJECTURE $1[12,14]$. Every hyperKähler variety $X$ admits a self-dual multiplicative Chow-Künneth decomposition such that its Chern classes lie in $\mathrm{CH}^{*}(X)_{0}$.

As a corollary to Theorem 2, one may state decomposition theorems for families of varieties that belong to the set $E$ described in Theorem 2 as those first stated by Voisin [15] for families of K3 surfaces. One may also consult [14] for the case of the relative Hilbert scheme of length- $n$ subschemes on a family of K3 surfaces.

1.1. Outline. We start in Section 2 by showing that the property of having a self-dual Chow-Künneth decomposition is stable under the following four operations: product, projective bundle, blow-up, and finite quotient by a group action. In Section 3, we show under suitable conditions (mostly concerning Chern classes) that the property of having a self-dual multiplicative Chow-Künneth decomposition is stable under the same four operations. Propositions 3.3 and 3.4 complement [12, Propositions 13.1 and 13.2] where the self-duality assumption was overlooked. We then want to use these general results to show that a certain desingularization of the map $X^{3} \rightarrow X^{[3]}$ has a self-dual multiplicative ChowKünneth decomposition. Since one needs to blow up $X^{3}$ several times in order to resolve the map $X^{3} \rightarrow X^{[3]}$, it is convenient to state a proposition that takes care of the centers of the successive blow-ups all at once, so that we do not have to check the assumptions of Proposition 3.4 at each step. This is the content of Section 4 and the main result there is Proposition 4.4. In Section 5, we resolve explicitly the rational map $X^{3} \rightarrow X^{[3]}$ into a morphism $p: X_{3} \rightarrow X^{[3]}$ by blowing 
up $X^{3}$ along subvarieties that are stable under the action of the symmetric group $\mathfrak{S}_{3}$ which permutes the factors, and we show that $p$ factors through the quotient morphism and then contracts two irreducible divisors (these contractions are not smooth blow-up morphisms). In Section 6, we equip $X_{3}$ with a $\mathfrak{S}_{3}$-invariant selfdual multiplicative Chow-Künneth decomposition, show that it descends along the resolution $p: X_{3} \rightarrow X^{[3]}$, and prove Theorem 1 . A technical difficulty consists in showing that the correspondence ${ }^{t} \Gamma_{p} \circ \Gamma_{p}$ sits in $\mathrm{CH}^{3 d}\left(X_{3} \times X_{3}\right)_{0}$, which in turn requires the careful analysis, carried out in Section 5, of the morphism $X_{3} \rightarrow X^{[3]}$. One should note that for our purpose of constructing idempotents in the ring of correspondences, it is not sufficient to show, for example, that $p^{*} p_{*}$ preserves the grading on $X_{3}$ to conclude; it is really necessary to work at the level of correspondences, which yet again adds a level of technicality. Finally, in Section 7, we prove the analogue of Theorem 1 for the nested Hilbert schemes $X^{[1,2]}$ and $X^{[2,3]}$.

For the sake of simplicity, we have not considered the question of constructing a Chow-Künneth decomposition for $X^{[3]}$ if $X$ is only assumed to be endowed with a Chow-Künneth decomposition (not necessarily self-dual or multiplicative). The main reason is that while Chow-Künneth idempotents are central in the ring of correspondences modulo numerical equivalence, this is far from being the case modulo rational equivalence. In fact, a correspondence commutes with the Chow-Künneth projectors if and only if it sits in the graded-0 part of the ring of correspondences (see Lemma 2.5). Therefore, if one assumes the Chow-Künneth decomposition to be multiplicative, then one can keep track of intersections and compositions of correspondences that sit in grade 0 , thus making the arguments simpler. Nevertheless, our main theorem adds to the restricted class of varieties that can be endowed with a multiplicative Chow-Künneth decomposition. That such a property is stable by the not-so-simple operation of taking the Hilbert cube is intriguing.

1.2. Conventions. Chow groups are always understood with rational coefficients. The Chow motive of a smooth projective variety $X$ is denoted $\mathfrak{h}(X)$. Following Voevodsky, we think of the theory of motives as a homology theory. Consequently, the functor $\mathfrak{h}$ is seen as a covariant functor, and correspondences act covariantly. Moreover, denoting $\mathbb{1}:=\mathfrak{h}(\operatorname{Spec} k)$, we set the Tate twists to follow the convention that $\mathfrak{h}\left(\mathbb{P}^{1}\right)=\mathbb{1} \oplus \mathbb{1}(1)$.

\section{Self-dual Chow-Künneth decompositions}

DEFINITION 2.1. Let $X$ be a smooth projective variety of dimension $d$ over a field $k$, endowed with a Chow-Künneth decomposition $\left\{\pi_{X}^{i}: 0 \leqslant i \leqslant 2 d\right\}$. The 
Chow-Künneth decomposition $\left\{\pi_{X}^{i}: 0 \leqslant i \leqslant 2 d\right\}$ is said to be self-dual if

$$
{ }^{t} \pi^{i}=\pi^{2 d-i} \in \mathrm{CH}^{d}(X \times X) \text { for all } 0 \leqslant i \leqslant 2 d .
$$

Here, given a correspondence $\Gamma \in \mathrm{CH}^{*}(X \times X)$, the transpose denoted ${ }^{t} \Gamma$ is the correspondence obtained from $\Gamma$ by switching the two factors of $X \times X$.

It is well known that operations on varieties such as taking products, projective bundles and blow-ups preserve the property of having a ChowKünneth decomposition. In addition to recalling these results (and to setting up notations along the way), we also give a sufficient condition for a ChowKünneth decomposition to descend along a generically finite morphism. The principal goal of this section is to show that the property that the Chow-Künneth decompositions are self-dual is also preserved under these operations as well as under the operation of taking a generically finite quotient.

2.1. Product varieties. Recall that, given a smooth projective variety $X$ endowed with a Chow-Künneth decomposition $\left\{\pi_{X}^{i}\right\}$, we have defined in (2) the graded pieces

$$
\mathrm{CH}^{i}(X)_{s}:=\left(\pi_{X}^{2 i-s}\right)_{*} \mathrm{CH}^{i}(X) .
$$

Let $X$ and $Y$ be smooth projective varieties endowed with Chow-Künneth decompositions $\left\{\pi_{X}^{i}\right\}$ and $\left\{\pi_{Y}^{i}\right\}$, respectively. Thanks to the Künneth formula, the idempotents

$$
\pi_{X \times Y}^{i}:=\sum_{i=i_{1}+i_{2}} \pi_{X}^{i_{1}} \otimes \pi_{Y}^{i_{2}}
$$

define a Chow-Künneth decomposition for the product variety $X \times Y$. Here and throughout this work, given any two correspondences $\alpha \in \mathrm{CH}^{*}\left(X_{1} \times X_{2}\right)$ and $\beta \in \mathrm{CH}^{*}\left(Y_{1} \times Y_{2}\right)$, we define the correspondence

$$
\alpha \otimes \beta:=p_{13}^{*} \alpha \cdot p_{24}^{*} \beta \in \mathrm{CH}^{*}\left(\left(X_{1} \times Y_{1}\right) \times\left(X_{2} \times Y_{2}\right)\right),
$$

where $p_{i j}$ is the projector from $X_{1} \times Y_{1} \times X_{2} \times Y_{2}$ on the product of the $i$ th and $j$ th factors. With the above product Chow-Künneth decomposition, we have

$$
p_{1}^{*} \mathrm{CH}^{p}(X)_{s} \cdot p_{2}^{*} \mathrm{CH}^{q}(Y)_{t} \subseteq \mathrm{CH}^{p+q}(X \times Y)_{s+t} ;
$$

see [12, Proposition 8.7].

DEFINITION 2.2. A correspondence $\Gamma \in \mathrm{CH}^{p}(X \times Y)$ is said to be of pure grade $s$ if

$$
\Gamma \in \mathrm{CH}^{p}(X \times Y)_{s} .
$$


In particular, a morphism $f: X \rightarrow Y$ is of pure grade 0 if its graph is in $\mathrm{CH}^{d_{Y}}(X \times$ $Y)_{0}$, where $d_{Y}=\operatorname{dim} Y$.

REMARK 2.3. The correspondence $\Gamma \in \mathrm{CH}^{p}(X \times Y)$ is of pure grade $s$ if and only if

$$
\left(\pi_{X}^{i} \otimes \pi_{Y}^{j}\right)_{*} \Gamma=0 \quad \text { for all pairs } i+j \neq 2 p-s .
$$

Indeed, $\Gamma \in \mathrm{CH}^{p}(X \times Y)$ is of pure grade $s$ if and only if $\left(\pi_{X \times Y}^{k}\right)_{*} \Gamma=0$ for any $k \neq 2 p-s$, and then use definition (3).

Now we turn to self-dual Chow-Künneth decompositions. The following proposition is clear:

Proposition 2.4. Assume that $X$ and $Y$ are endowed with the action of a finite group $G$. If $\left\{\pi_{X}^{i}\right\}$ and $\left\{\pi_{Y}^{i}\right\}$ are $G$-invariant and self-dual, then $\left\{\pi_{X \times Y}^{i}\right\}$ is $G$ invariant and self-dual.

The following lemma is a criterion for a correspondence to be of pure grade.

LEMMA 2.5. Let $X$ and $Y$ be smooth projective varieties endowed with self-dual Chow-Künneth decompositions $\left\{\pi_{X}^{i}: 0 \leqslant i \leqslant 2 d_{X}\right\}$ and $\left\{\pi_{Y}^{j}: 0 \leqslant j \leqslant 2 d_{Y}\right\}$, where $d_{X}=\operatorname{dim} X$ and $d_{Y}=\operatorname{dim} Y$. Let $\Gamma \in \mathrm{CH}^{p}(X \times Y)$ be a correspondence. Then $\Gamma$ is of pure grade $s$, namely $\Gamma \in \mathrm{CH}^{p}(X \times Y)_{s}$, if and only if it satisfies

$$
\pi_{Y}^{i} \circ \Gamma=\Gamma \circ \pi_{X}^{2\left(d_{X}-p\right)+s+i}, \quad \text { for all } i .
$$

In particular, a self-correspondence $\Gamma \in \mathrm{CH}^{d_{X}}(X \times X)$ is of pure grade 0 if and only if it commutes with the Chow-Künneth projectors $\pi_{X}^{i}$, namely

$$
\pi_{X}^{i} \circ \Gamma=\Gamma \circ \pi_{X}^{i}, \quad \text { for all } 0 \leqslant i \leqslant 2 d_{X} .
$$

Proof. By Remark 2.3, if the correspondence $\Gamma$ lies in $\mathrm{CH}^{d}(X \times Y)_{s}$ then

$$
\begin{aligned}
\pi_{Y}^{i} \circ \Gamma \circ \pi_{X}^{2\left(d_{X}-p\right)+s+i} & =\left({ }^{t} \pi_{X}^{2\left(d_{X}-p\right)+s+i} \otimes \pi_{Y}^{i}\right)_{*} \Gamma \\
& =\left(\pi_{X}^{2 p-s-i} \otimes \pi_{Y}^{i}\right)_{*} \Gamma \\
& =\left(\left(\sum_{j=0}^{2 d_{X}} \pi_{X}^{j}\right) \otimes \pi_{Y}^{i}\right)_{*} \Gamma \\
& =\left(\Delta_{X} \otimes \pi_{Y}^{i}\right)_{*} \Gamma \\
& =\pi_{Y}^{i} \circ \Gamma,
\end{aligned}
$$


for all $0 \leqslant i \leqslant 2 d_{Y}$. Note that here we used the fact that $\left\{\pi_{X}^{i}\right\}$ is self-dual in an essential way. A similar computation yields

$$
\begin{aligned}
\pi_{Y}^{i} \circ \Gamma \circ \pi_{X}^{2\left(d_{X}-p\right)+s+i} & =\left(\pi_{X}^{2 p-s-i} \otimes\left(\sum_{j=0}^{2 d_{Y}} \pi_{Y}^{j}\right)\right)_{*} \Gamma \\
& =\left(\pi_{X}^{2 p-s-i} \otimes \Delta_{Y}\right)_{*} \Gamma=\Gamma \circ \pi_{X}^{2\left(d_{X}-p\right)+s+i} .
\end{aligned}
$$

Thus $\pi_{Y}^{i} \circ \Gamma=\Gamma \circ \pi_{X}^{2\left(d_{X}-p\right)+s+i}$. Conversely, if the above equality holds, then

$$
\left(\pi_{X}^{2 p-s-j} \otimes \pi_{Y}^{i}\right)_{*} \Gamma=\pi_{Y}^{i} \circ \Gamma \circ \pi_{X}^{2\left(d_{X}-p\right)+s+j}=\pi_{Y}^{i} \circ \pi_{Y}^{j} \circ \Gamma=0
$$

for all $i \neq j$. By Remark 2.3, $\Gamma$ lies in $\mathrm{CH}^{p}(X \times Y)_{s}$.

The action of a correspondence of pure grade shifts the grading.

Proposition 2.6. Let $X, Y, X^{\prime}$ and $Y^{\prime}$ be smooth projective varieties with selfdual Chow-Künneth decomposition. Then the following are true.

(i) Let $Z$ be a smooth projective variety with a Chow-Künneth decomposition $\left\{\pi_{Z}^{i}\right\}$ which is not necessarily multiplicative or self-dual. Let $\Gamma \in \mathrm{CH}^{p}(X \times$ $Y)$ be of pure grade s, that is, $\Gamma \in \mathrm{CH}^{p}(X \times Y)_{s}$, then

$$
(\Gamma \otimes \mathrm{id})_{*}: \mathrm{CH}^{l}(X \times Z)_{r} \rightarrow \mathrm{CH}^{l+p-d_{X}}(Y \times Z)_{r+s} .
$$

In particular, the pull-back and push-forward by a morphism of pure grade 0 preserves the gradings on the Chow groups.

(ii) If $\Gamma \in \mathrm{CH}^{*}(X \times Y)$ is of pure grade $s$ and $\Gamma^{\prime} \in \mathrm{CH}^{*}\left(Y \times Y^{\prime}\right)$ is of pure grade $s^{\prime}$, then $\Gamma^{\prime} \circ \Gamma \in \mathrm{CH}^{*}\left(X \times Y^{\prime}\right)$ is of pure grade $s+s^{\prime}$.

(iii) If $\Gamma \in \mathrm{CH}^{*}(X \times Y)$ and $\Gamma^{\prime} \in \mathrm{CH}^{*}\left(X^{\prime} \times Y^{\prime}\right)$ are of pure grades $s$ and $s^{\prime}$ respectively, then $\Gamma \otimes \Gamma^{\prime} \in \mathrm{CH}\left(X \times X^{\prime} \times Y \times Y^{\prime}\right)$ is of pure grade $s+s^{\prime}$.

Proof. To prove (i), we first apply Lemma 2.5 and get $\pi_{Y}^{i} \circ \Gamma=\Gamma \circ \pi_{X}^{2\left(d_{X}-p\right)+s+i}$. After tensoring with the identity correspondence of $Z$ we get

$$
\left(\pi_{Y}^{i} \otimes \pi_{Z}^{j}\right) \circ\left(\Gamma \otimes \mathrm{id}_{Z}\right)=\left(\Gamma \otimes \mathrm{id}_{Z}\right) \circ\left(\pi_{X}^{2\left(d_{X}-p\right)+s+i} \otimes \pi_{Z}^{j}\right) .
$$

If $\alpha \in \mathrm{CH}^{l}(X \times Z)_{r}$, then the above identity implies $\left(\pi_{Y}^{i} \otimes \pi_{Z}^{j}\right)_{*}\left(\Gamma \otimes \mathrm{id}_{Z}\right)_{*} \alpha=\left(\Gamma \otimes \mathrm{id}_{Z}\right)_{*}\left(\pi_{X}^{2\left(d_{X}-p\right)+s+i} \otimes \pi_{Z}^{j}\right)_{*} \alpha=\left(\Gamma \otimes \mathrm{id}_{Z}\right)_{*} 0=0$, for all $2\left(d_{X}-p\right)+s+i+j \neq 2 l-r$. This precisely means that

$$
\left(\Gamma \otimes \mathrm{id}_{Z}\right)_{*} \alpha \in \mathrm{CH}^{l+p-d_{X}}(Y \times Z)_{r+s} .
$$

Statements (ii) and (iii) are proved similarly using Lemma 2.5 . 
Note that a Chow-Künneth decomposition being self-dual implies that the diagonal is of pure grade 0 . One consequence of this fact is the following proposition.

COROLlaRY 2.7. Let $X$ and $Y$ be two smooth projective varieties endowed with self-dual Chow-Künneth decompositions. Then the two natural projections $p_{1}$ : $X \times Y \rightarrow X$ and $p_{2}: X \times Y \rightarrow Y$ are of pure grade 0 . Furthermore,

$$
p_{1_{*}} \mathrm{CH}^{p}(X \times Y)_{s} \subseteq \mathrm{CH}^{p-d_{Y}}(X)_{s}, \quad p_{2_{*}} \mathrm{CH}^{p}(X \times Y)_{s} \subseteq \mathrm{CH}^{p-d_{X}}(Y)_{s} .
$$

Proof. Note that $\Gamma_{p_{1}}=p_{13}^{*} \Delta_{X} \in \mathrm{CH}^{d_{X}}(X \times Y \times X)$, where $p_{13}: X \times Y \times X \rightarrow$ $X \times X$ is the projection onto the product of the first and the third factors. By (5) and the fact that $\Delta_{X} \in \mathrm{CH}^{d_{X}}(X \times X)_{0}$, we conclude that $\Gamma_{p_{1}} \in \mathrm{CH}^{d_{X}}(X \times Y \times X)_{0}$, namely $p_{1}$ is of pure grade 0 . One similarly shows that $p_{2}$ is also of pure grade 0 . The action on Chow groups follows immediately from Proposition 2.6.

2.2. Projective bundles. Let $X$ be a smooth projective variety of dimension $d$ and let $\mathscr{E}$ be a vector bundle on $X$ of rank $r+1$. Denote $\pi: \mathbb{P}(\mathscr{E}) \rightarrow X$ the geometric projectivization of $\mathscr{E}$. We define

$$
\gamma_{0}:=c_{r}\left(\pi^{*} \mathscr{E} / \mathcal{O}(-1)\right)=\sum_{i=0}^{r} \pi^{*} c_{i}(\mathscr{E}) \xi^{r-i},
$$

where $\xi \in \mathrm{CH}^{1}(\mathbb{P}(\mathscr{E}))$ is the first Chern class of $\mathcal{O}(1)$; then $\gamma_{0}$ satisfies $\xi \cdot \gamma_{0}=$ $-\pi^{*} c_{r+1}(\mathscr{E})$. Consider the correspondence

$$
\gamma:=\iota_{*} \gamma_{0} \in \mathrm{CH}^{2 r+d}(\mathbb{P}(\mathscr{E}) \times \mathbb{P}(\mathscr{E})),
$$

where $\iota: \mathbb{P}(\mathscr{E}) \hookrightarrow \mathbb{P}(\mathscr{E}) \times \mathbb{P}(\mathscr{E})$ is the diagonal embedding of $\mathrm{P}(\mathscr{E})$; its action consists in intersecting with $\gamma_{0}$. It has been known since Manin [8] that the Chow motive of $\mathbb{P}(\mathscr{E})$ is isomorphic to $\bigoplus_{l=0}^{r} \mathfrak{h}(X)(l)$. In fact an isomorphism is given by the correspondence (see the proof of Proposition 2.8)

$$
\Phi:=\left(\bigoplus_{l=0}^{r-1} h^{l} \circ{ }^{t} \Gamma_{\pi}\right) \oplus \gamma \circ{ }^{t} \Gamma_{\pi}:\left(\bigoplus_{l=0}^{r-1} \mathfrak{h}(X)(l)\right) \oplus \mathfrak{h}(X)(r) \longrightarrow \mathfrak{h}(\mathbb{P}(\mathscr{E})),
$$

which induces an isomorphism:

$\Phi_{*}: \bigoplus_{l=0}^{r} \mathrm{CH}^{p-l}(X) \stackrel{\cong}{\longrightarrow} \mathrm{CH}^{p}(\mathbb{P}(\mathscr{E})), \quad\left(\beta_{0}, \ldots, \beta_{r}\right) \mapsto \sum_{l=0}^{r-1} \xi^{l} \cdot \pi^{*} \beta_{l}+\gamma_{0} \cdot \pi^{*} \beta_{r}$ 
Here, for a morphism of varieties $g: V \rightarrow W$ we denote $\Gamma_{g} \in \mathrm{CH}_{\operatorname{dim} V}(V \times W)$ the class of the graph $\{(x, g(x)): x \in V\}$, and $h^{l} \in \mathrm{CH}^{d+r+l}(\mathbb{P}(\mathscr{E}) \times \mathbb{P}(\mathscr{E}))$ the push-forward of $\xi^{l} \in \mathrm{CH}^{l}(\mathbb{P}(\mathscr{E}))$ under the diagonal embedding $\iota$ (so that $h^{l}$ is the $l$ th power of $h$ as a correspondence, rather than as an algebraic cycle). This isomorphism is not quite the usual projective bundle formula isomorphism $\bigoplus_{l=0}^{r} \mathrm{CH}^{p-l}(X) \stackrel{\cong}{\longrightarrow} \mathrm{CH}^{p}(\mathbb{P}(\mathscr{E})),\left(\beta_{0}, \ldots, \beta_{r}\right) \mapsto \sum_{l=0}^{r} \xi^{l} \cdot \pi^{*} \beta_{l}$. The usual projective bundle isomorphism suffices to get all results of this section. The reason for modifying the last summand is to make it compatible with the corresponding isomorphism for a smooth blow-up; see Section 2.3. This compatibility is needed in Remark 3.6 to get a commutative diagram.

Of course, if $X$ has a Chow-Künneth decomposition $\left\{\pi_{X}^{i}\right\}$, then the isomorphism $\Phi$ induces a Chow-Künneth decomposition for $\mathbb{P}(\mathscr{E})$, namely

$$
p_{\mathrm{P}(\mathscr{E})}^{i}:=\Phi \circ\left(\bigoplus_{j=0}^{r} \pi_{X}^{i-2 j}\right) \circ \Phi^{-1} .
$$

However, a variety can be endowed with many different Chow-Künneth decompositions in general and Manin's isomorphism may not preserve certain properties of Chow-Künneth decompositions: if $\left\{\pi_{X}^{i}\right\}$ is self-dual, then $\left\{p_{\mathbb{P}(\mathscr{E})}^{i}\right\}$ may not be self-dual. The following proposition shows that if $X$ has a selfdual Chow-Künneth decomposition, then $\mathbb{P}(\mathscr{E})$ has a self-dual Chow-Künneth decomposition as well.

Proposition 2.8. Let $X$ be a smooth projective variety endowed with the action of a finite group $G$. Assume that $X$ has a G-invariant self-dual Chow-Künneth decomposition $\left\{\pi_{X}^{i}\right\}$. If $\mathscr{E} \rightarrow X$ is a $G$-equivariant vector bundle on $X$ of rank $r+1$, then the geometric projectivization $\mathbb{P}(\mathscr{E})$ has a G-invariant self-dual ChowKünneth decomposition $\left\{\pi_{\mathbb{P}(\mathscr{E})}^{i}\right\}$.

Proof. The correspondence ${ }^{t} \Phi$ defines a morphism of motives

$$
{ }^{t} \Phi: \mathfrak{h}(\mathbb{P}(\mathscr{E})) \longrightarrow \mathfrak{h}(X)(r) \oplus \mathfrak{h}(X)(r-1) \oplus \cdots \oplus \mathfrak{h}(X)
$$

\section{Let}

$\sigma: \mathfrak{h}(X)(r) \oplus \mathfrak{h}(X)(r-1) \oplus \cdots \oplus \mathfrak{h}(X) \longrightarrow \mathfrak{h}(X) \oplus \mathfrak{h}(X)(1) \oplus \cdots \oplus \mathfrak{h}(X)(r)$

be the morphism that permutes the direct summands. This morphism is self-dual in the sense that

$$
{ }^{t} \sigma=\sigma
$$


The correspondence $\Gamma_{\pi} \circ h^{l} \circ{ }^{t} \Gamma_{\pi}$ vanishes for $l<r$ and is equal to $\Delta_{X}$ for $l=r$ (see for instance [13, Section 1]). The identity $\xi \cdot \gamma_{0}=-\pi^{*} c_{r+1}(\mathscr{E})$ implies that the correspondence $\Gamma_{\pi} \circ h^{l} \circ \gamma \circ{ }^{t} \Gamma_{\pi}$ vanishes for $1 \leqslant l \leqslant r$ and equals $\Delta_{X}$ for $l=0$. Hence

$$
\begin{aligned}
\Gamma_{\pi} \circ \gamma \circ \gamma \circ{ }^{t} \Gamma_{\pi} & =\sum_{i=0}^{r} \Gamma_{\pi} \circ\left(\cdot \xi^{i} \pi^{*} c_{r-i}(\mathscr{E})\right) \circ \gamma \circ{ }^{t} \Gamma_{\pi} \\
& =\sum_{i=0}^{r} \Gamma_{\pi} \circ h^{i} \circ \gamma \circ{ }^{t} \Gamma_{\pi} \circ\left(\cdot c_{r-i}(\mathscr{E})\right) \\
& =\cdot c_{r}(\mathscr{E}) .
\end{aligned}
$$

Here, we used the following notation. If $\alpha$ is a cycle in $\mathrm{CH}^{p}(Y)$, then $\cdot \alpha$ is the correspondence defined by $\left(\iota_{Y}\right)_{*} \alpha \in \mathrm{CH}^{p+d_{Y}}(Y \times Y)$ where $\iota_{Y}: Y \hookrightarrow Y \times Y$ is the diagonal embedding. Then it is easy to see that we have the identity

$$
\sigma \circ{ }^{t} \Phi \circ \Phi=\left(\begin{array}{ccccc}
\Delta_{X} & 0 & \cdots & 0 & 0 \\
0 & \Delta_{X} & \cdots & 0 & 0 \\
0 & * & \ddots & 0 & 0 \\
0 & * & \cdots & \Delta_{X} & 0 \\
c_{r}(\mathscr{E}) & 0 & \cdots & 0 & \Delta_{X}
\end{array}\right) .
$$

In other words, we can write

$$
\sigma \circ{ }^{t} \Phi \circ \Phi=\mathrm{id}+\eta
$$

for a correspondence $\eta$ that is nilpotent of index $r$, that is, satisfies $\eta^{\circ r}=0$. By taking the transpose of the above equation, we get ${ }^{t} \Phi \circ \Phi \circ \sigma=\mathrm{id}+{ }^{t} \eta$, and hence $\eta \circ \sigma=\sigma \circ{ }^{t} \eta$. ( $\eta$ should be thought of as a strict lower triangular matrix and $\sigma$ as being the operation of rotating the matrix by an angle of $\pi / 2$.) We define the correspondence

$$
\Psi:=\Phi \circ(\mathrm{id}+\eta)^{-1 / 2} .
$$

Here, for a nilpotent element $x$ of order $r$ and for any real number $a$, we formally set

$$
(1+x)^{a}=1+a x+\frac{a(a-1)}{2} x^{2}+\cdots+\frac{a(a-1) \cdots(a-r+2)}{(r-1) !} x^{r-1} .
$$

The correspondence $\Psi: \bigoplus_{l=0}^{r} \mathfrak{h}(X)(l) \longrightarrow \mathfrak{h}(\mathbb{P}(\mathscr{E}))$ is an isomorphism; its inverse is $(\mathrm{id}+\eta)^{1 / 2} \circ \Phi^{-1}$. In fact we have

$$
\sigma \circ{ }^{t} \Psi=\Psi^{-1}
$$


because

$$
\begin{aligned}
\sigma \circ{ }^{t} \Psi \circ \Psi & =\sigma \circ\left(\mathrm{id}+{ }^{t} \eta\right)^{-1 / 2} \circ{ }^{t} \Phi \circ \Phi \circ(\mathrm{id}+\eta)^{-1 / 2} \\
& =(\mathrm{id}+\eta)^{-1 / 2} \circ \sigma \circ{ }^{t} \Phi \circ \Phi \circ(\mathrm{id}+\eta)^{-1 / 2} \\
& =(\mathrm{id}+\eta)^{-1 / 2} \circ(\mathrm{id}+\eta) \circ(\mathrm{id}+\eta)^{1 / 2} \\
& =\mathrm{id} .
\end{aligned}
$$

It follows that

$$
\pi_{\mathrm{P}(\mathscr{E})}^{i}:=\Psi \circ\left(\bigoplus_{j=0}^{r} \pi_{X}^{i-2 j}\right) \circ \Psi^{-1}
$$

defines a Chow-Künneth decomposition of $\mathbb{P}(\mathscr{E})$ that is self-dual if $\left\{\pi_{X}^{i}\right\}$ is selfdual. Indeed, on the one hand, we have

$$
{ }^{t} \pi_{\mathrm{P}(\mathscr{E})}^{i}=\Psi \circ \sigma \circ\left(\pi_{X}^{2 d-i} \oplus \cdots \oplus \pi_{X}^{2 d+2 r-i}\right) \circ{ }^{t} \Psi,
$$

and on the other hand, we have

$$
\pi_{\mathbb{P}(\mathscr{E})}^{2 d+2 r-i}=\Psi \circ\left(\pi_{X}^{2 d+2 r-i} \oplus \cdots \oplus \pi_{X}^{2 d-i}\right) \circ \sigma \circ{ }^{t} \Psi,
$$

and clearly $\sigma \circ\left(\pi_{X}^{2 d-i} \oplus \cdots \oplus \pi_{X}^{2 d+2 r-i}\right)=\left(\pi_{X}^{2 d+2 r-i} \oplus \cdots \oplus \pi_{X}^{2 d-i}\right) \circ \sigma$.

The Chow-Künneth decomposition (8) is $G$-invariant if $\left\{\pi_{X}^{i}\right\}$ is $G$ invariant because $\Gamma_{\pi}$ is $G$-invariant (by assumption) and $h$ is $G$-invariant (the automorphism group of projective space preserves $\mathcal{O}(1)$ ).

REMARK 2.9. The construction of $\pi_{\mathrm{P}(\mathscr{E})}^{i}$ commutes with products. To be more precise, let $Y$ be any smooth projective variety with self-dual Chow-Künneth decomposition $\left\{\pi_{Y}^{j}\right\}$. The self-dual Chow-Künneth decomposition of $\mathbb{P}(\mathscr{E}) \times Y$, viewed as a projective bundle over $X \times Y$, agrees with the product Chow-Künneth decomposition.

2.3. Blow-ups. Let $X$ be a smooth projective variety and let $Y$ be smooth closed subvariety of codimension $r+1$ of $X$. It has been known since Manin [8] that the motive of the blow-up $\tilde{X}$ of $X$ along $Y$ can be expressed as

$$
\mathfrak{h}(\tilde{X}) \cong \mathfrak{h}(X) \oplus \mathfrak{h}(Y)(1) \oplus \cdots \oplus \mathfrak{h}(Y)(r) .
$$

Consequently, if $X$ and $Y$ have a Chow-Künneth decomposition then so does $\tilde{X}$.

The set-up for the proposition below is the following: $X$ is a smooth projective variety endowed with the action of a finite group $G$ and $i: Y \hookrightarrow X$ is a smooth 
closed subvariety of codimension $r+1$ such that $g \cdot Y=Y$ for all $g \in G$. The blow-up of $X$ along $Y$ is denoted $\tilde{X}$. We have the blow-up diagram

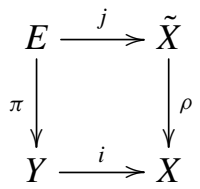

where $E \cong \mathbb{P}\left(\mathscr{N}_{Y / X}\right)$ is the exceptional divisor.

Proposition 2.10. In the situation above, if $X$ and $Y$ both have a $G$-invariant self-dual Chow-Künneth decomposition, namely $\left\{\pi_{X}^{i}\right\}$ and $\left\{\pi_{Y}^{i}\right\}$, then $\tilde{X}$ has a $G$-invariant self-dual Chow-Künneth decomposition $\left\{\pi_{\tilde{X}}^{i}\right\}$.

Proof. First note that by functoriality of blow-ups the action of $G$ on $X$ lifts to an action on $\tilde{X}$, and all maps involved in the diagram (9) are $G$-equivariant. Therefore, the corresponding push-forwards and pull-backs on Chow groups are also $G$-equivariant.

By Manin [8], the correspondence

$$
\Phi:={ }^{t} \Gamma_{\rho} \oplus \bigoplus_{l=1}^{r} \Gamma_{j} \circ h^{l-1} \circ{ }^{t} \Gamma_{\pi}: \mathfrak{h}(X) \oplus \bigoplus_{l=1}^{r} \mathfrak{h}(Y)(l) \longrightarrow \mathfrak{h}(\tilde{X})
$$

is an isomorphism of Chow motives. Here, $h:=-{ }^{t} \Gamma_{j} \circ \Gamma_{j}$; its action on $\mathrm{CH}_{*}(E)$ consists in intersecting with the first Chern class of the relative $\mathcal{O}(1)$-bundle $\left.\mathcal{O}_{\tilde{X}}(-E)\right|_{E}$. In fact, that $\Phi$ is an isomorphism is a consequence of Manin's identity principle coupled with the fact that $\Phi$ induces an isomorphism of Chow groups: the blow-up formula for Chow groups [6, Proposition 6.7(e)]

$$
\begin{gathered}
\Phi_{*}: \mathrm{CH}^{p}(X) \oplus\left(\bigoplus_{l=1}^{r} \mathrm{CH}^{p-l}(Y)\right) \stackrel{\cong}{\longrightarrow} \mathrm{CH}^{p}(\tilde{X}), \\
\left(\alpha, \beta_{1}, \ldots, \beta_{r}\right) \mapsto \rho^{*} \alpha+j_{*}\left(\sum_{l=1}^{r} \xi^{l-1} \cdot \pi^{*} \beta_{l}\right),
\end{gathered}
$$

where $\xi:=c_{1}\left(\left.\mathcal{O}_{\tilde{X}}(-E)\right|_{E}\right) \in \mathrm{CH}^{1}(E)$. A Chow-Künneth decomposition for $\tilde{X}$ is then given by

$$
p_{\tilde{X}}^{i}:=\Phi \circ\left(\pi_{X}^{i} \oplus \bigoplus_{j=1}^{r} \pi_{Y}^{i-2 j}\right) \circ \Phi^{-1} .
$$


(The correspondence $\Phi^{-1}$ is described explicitly in [13, Section 5].) Since the diagram (9) is $G$-equivariant, it is apparent that $\Phi$ is $G$-invariant. By assumption, $\left\{\pi_{X}^{i}\right\}$ and $\left\{\pi_{Y}^{i}\right\}$ are $G$-invariant. It follows that $\left\{p_{\tilde{X}}^{i}\right\}$ is $G$-invariant.

Let us now assume that $\left\{\pi_{X}^{i}\right\}$ and $\left\{\pi_{Y}^{i}\right\}$ are both self-dual. The Chow-Künneth decomposition $\left\{p_{\tilde{X}}^{i}\right\}$ constructed above is not self-dual in general. Here is a way to make it self-dual while preserving its $G$-invariance. Let

$$
\sigma: \mathfrak{h}(X) \oplus \mathfrak{h}(Y)(r) \oplus \cdots \oplus \mathfrak{h}(Y)(1) \longrightarrow \mathfrak{h}(X) \oplus \mathfrak{h}(Y)(1) \oplus \cdots \oplus \mathfrak{h}(Y)(r)
$$

be the morphism that switches the summands. By [13, Lemma 5.2], the correspondence $\sigma \circ{ }^{t} \Phi \circ \Phi$ from $\mathfrak{h}(X) \oplus \bigoplus_{i=1}^{r} \mathfrak{h}(Y)(i)$ to itself can be written in matrix form as a lower triangular matrix:

$$
\sigma \circ{ }^{t} \Phi \circ \Phi=\left(\begin{array}{ccccc}
\Delta_{X} & 0 & 0 & \cdots & 0 \\
0 & -\Delta_{Y} & 0 & \cdots & 0 \\
0 & * & \ddots & \ddots & \vdots \\
\vdots & \vdots & \ddots & \ddots & 0 \\
0 & * & \cdots & * & -\Delta_{Y}
\end{array}\right) .
$$

Let us write $\sigma \circ{ }^{t} \Phi \circ \Phi=D \circ(\mathrm{id}+\eta)$, where $D$ is the diagonal matrix with first diagonal entry $\Delta_{X}$ and remaining diagonal entries $-\Delta_{Y}$. Note that $\sigma={ }^{t} \sigma$ and that $\sigma$ commutes with $D$. The correspondence $\eta$ is clearly nilpotent of index $r$ and commutes with $D$; we also have $\eta \circ \sigma=\sigma \circ{ }^{t} \eta$. We define

$$
\Psi:=\Phi \circ(\mathrm{id}+\eta)^{-1 / 2} \text {. }
$$

Clearly, $\Psi$ is an isomorphism with inverse $(\mathrm{id}+\eta)^{1 / 2} \circ \Phi^{-1}$. We claim that in fact

$$
D \circ \sigma \circ{ }^{t} \Psi=\Psi^{-1} \text {. }
$$

Indeed, we have

$$
\begin{aligned}
D \circ \sigma \circ{ }^{t} \Psi \circ \Psi & =D \circ \sigma \circ\left(\mathrm{id}+{ }^{t} \eta\right)^{-1 / 2} \circ{ }^{t} \Phi \circ \Phi \circ(\mathrm{id}+\eta)^{-1 / 2} \\
& =(\mathrm{id}+\eta)^{-1 / 2} \circ D \circ \sigma \circ \sigma \circ D \circ(\mathrm{id}+\eta)^{1 / 2} \\
& =\mathrm{id},
\end{aligned}
$$

where we have used that $\eta \circ D \circ \sigma=D \circ \sigma \circ{ }^{t} \eta$ and that ${ }^{t} \Phi \circ \Phi=\sigma \circ D \circ(\mathrm{id}+\eta)$. Thus if one sets

$$
\pi_{\tilde{X}}^{i}:=\Psi \circ\left(\pi_{X}^{i} \oplus \bigoplus_{j=1}^{r} \pi_{Y}^{i-2 j}\right) \circ \Psi^{-1},
$$

then, as in the proof of Proposition 2.8, we see that $\left\{\pi_{\tilde{X}}^{i}\right\}$ defines a self-dual Chow-Künneth decomposition for $\tilde{X}$. Moreover, it is $G$-invariant because the maps involved in the diagram (9) are $G$-equivariant. 
REMARK 2.11. The proposition can be easily generalized to the case where $Y$ is a $G$-invariant disjoint union of smooth closed subvarieties of $X$. The construction of $\pi_{\tilde{X}}^{i}$ also commutes with taking product. Namely, if $X^{\prime}$ is another smooth projective variety with self-dual Chow-Künneth decomposition, then $\tilde{X} \times X^{\prime}$ is the blow-up of $X \times X^{\prime}$ with center $Y \times X^{\prime}$. Repeating the above construction in this setting, we obtain a self-dual Chow-Künneth decomposition on $\tilde{X} \times X^{\prime}$ that agrees with the product Chow-Künneth decomposition.

2.4. Generically finite quotients. Consider a surjective morphism $f: V \rightarrow$ $W$ of smooth projective varieties. The morphism $\Gamma_{f}: \mathfrak{h}(V) \rightarrow \mathfrak{h}(W)$ of motives is surjective and admits a section, say $s$. Thus $\mathfrak{h}(W)$ is isomorphic to the direct summand $\operatorname{Im}\left(s \circ \Gamma_{f}\right)$ of $\mathfrak{h}(V)$. Assume that $V$ has a Chow-Künneth decomposition $\left\{\pi_{V}^{i}\right\}$. Although it is true that the homology classes of the cycles $\Gamma_{f} \circ \pi_{V}^{i} \circ s$ give a Künneth decomposition for $V$ (because the idempotents $\pi_{V}^{i}$ are central modulo homological equivalence in the ring of self-correspondences of $V$ ), it is in general not true that the cycles $\Gamma_{f} \circ \pi_{V}^{i} \circ s$ define idempotents modulo rational equivalence. Thus finding a Chow-Künneth decomposition for $W$ is usually not a straightforward matter. However, Proposition 2.12 below gives a sufficient condition for a Chow-Künneth decomposition to descend along a generically finite morphism.

Proposition 2.12. Let $p: X \rightarrow Y$ be a generically finite morphism between smooth projective varieties of dimension $d$. Assume that $X$ is endowed with a selfdual Chow-Künneth decomposition $\left\{\pi_{X}^{i}\right\}$. If ${ }^{t} \Gamma_{p} \circ \Gamma_{p}$ sits in $\mathrm{CH}_{d}(X \times X)_{0}$, then $Y$ also has a self-dual Chow-Künneth decomposition given by

$$
\pi_{Y}^{i}=\frac{1}{N} \Gamma_{p} \circ \pi_{X}^{i} \circ{ }^{t} \Gamma_{p}, \quad 0 \leqslant i \leqslant 2 d,
$$

where $N$ is the degree of $p$. Furthermore the morphism $p$ is of pure grade 0.

Proof. It is clear that

$$
\Gamma_{p} \circ{ }^{t} \Gamma_{p}=N \Delta_{Y}
$$

It follows that the cycles $\pi_{Y}^{i}$ given in the proposition lift the Künneth components in cohomology. For all $i, j$, we have

$$
\begin{aligned}
N^{2} \pi_{Y}^{i} \circ \pi_{Y}^{j} & =\Gamma_{p} \circ \pi_{X}^{i} \circ\left({ }^{t} \Gamma_{p} \circ \Gamma_{p}\right) \circ \pi_{X}^{j} \circ{ }^{t} \Gamma_{p} \\
& =\Gamma_{p} \circ\left({ }^{t} \Gamma_{p} \circ \Gamma_{p}\right) \circ \pi_{X}^{i} \circ \pi_{X}^{j} \circ{ }^{t} \Gamma_{p} \\
& =N \Gamma_{p} \circ \pi_{X}^{i} \circ \pi_{X}^{j} \circ{ }^{t} \Gamma_{p}
\end{aligned}
$$




$$
= \begin{cases}N^{2} \pi_{Y}^{i}, & i=j \\ 0, & i \neq j .\end{cases}
$$

Here the second equality follows from Lemma 2.5. Hence $\left\{\pi_{Y}^{i}\right\}$ is a ChowKünneth decomposition and it is clearly self-dual.

To show that $p$ is of grade 0 , we note that if $i+j \neq 2 d$ then

$$
\begin{aligned}
\left(\pi_{X}^{i} \otimes \pi_{Y}^{j}\right)_{*} \Gamma_{p} & =\pi_{Y}^{j} \circ \Gamma_{p} \circ{ }^{t} \pi_{X}^{i} \\
& =\frac{1}{N} \Gamma_{p} \circ \pi_{X}^{j} \circ{ }^{t} \Gamma_{p} \circ \Gamma_{p} \circ \pi_{X}^{2 d-i} \\
& =\frac{1}{N} \Gamma_{p} \circ{ }^{t} \Gamma_{p} \circ \Gamma_{p} \circ \pi_{X}^{j} \circ \pi_{X}^{2 d-i} \\
& =0 .
\end{aligned}
$$

Hence $\Gamma_{p} \in \mathrm{CH}^{d}(X \times Y)_{0}$.

One application of independent interest of the above proposition concerns finite quotients.

COROLlary 2.13. Let $X$ be a smooth projective variety endowed with the action of a finite group $G$. Assume that $X$ has a $G$-invariant self-dual ChowKünneth decomposition $\left\{\pi_{X}^{i}\right\}$. Then the quotient variety $X / G$ has a self-dual Chow-Künneth decomposition $\left\{\pi_{X / G}^{i}\right\}$.

Proof. First, note that we do not need to assume that the quotient $X / G$ is smooth because we work with Chow groups with rational coefficients. For any element $g \in G$, let us still denote $g \in \mathrm{CH}_{d}(X \times X)$ the class of the graph of the action of $g$ on $X$. By Lemma 2.5, the Chow-Künneth decomposition $\left\{\pi_{X}^{i}\right\}$ is $G$-invariant if and only if $g \in \mathrm{CH}^{d}(X \times X)_{0}$, for all $g \in G$. Let $p: X \rightarrow Y=X / G$ be the quotient morphism. Then

$$
{ }^{t} \Gamma_{p} \circ \Gamma_{p}=\sum_{g \in G} g \in \mathrm{CH}^{d}(X \times X)_{0}
$$

and hence Proposition 2.12 applies, showing that

$$
\pi_{X / G}^{i}:=\frac{1}{|G|} \Gamma_{p} \circ \pi_{X}^{i} \circ{ }^{t} \Gamma_{p}
$$

defines a self-dual Chow-Künneth decomposition of $X / G$. 


\section{Multiplicative Chow-Künneth decompositions}

DEFINITION 3.1. Let $X$ be a smooth projective variety of dimension $d$ over a field $k$, endowed with a Chow-Künneth decomposition $\left\{\pi_{X}^{i}: 0 \leqslant i \leqslant 2 d\right\}$. The Chow-Künneth decomposition $\left\{\pi_{X}^{i}, 0 \leqslant i \leqslant 2 d\right\}$ is multiplicative if

$$
\pi_{X}^{k} \circ \Delta_{123} \circ\left(\pi_{X}^{i} \otimes \pi_{X}^{j}\right)=0 \in \mathrm{CH}^{2 d}(X \times X \times X) \quad \text { whenever } k \neq i+j .
$$

In particular, a multiplicative Chow-Künneth decomposition induces a multiplicative bi-grading on the Chow ring of $X$ :

$$
\mathrm{CH}^{*}(X)=\bigoplus_{i, s} \mathrm{CH}^{i}(X)_{s}, \quad \text { where } \mathrm{CH}^{i}(X)_{s}:=\left(\pi_{X}^{2 i-s}\right)_{*} \mathrm{CH}^{i}(X) .
$$

Note that $\pi_{X}^{k} \circ \Delta_{123} \circ\left(\pi_{X}^{i} \otimes \pi_{X}^{j}\right)=\left(\pi_{X}^{k} \otimes{ }^{t} \pi_{X}^{i} \otimes{ }^{t} \pi_{X}^{j}\right)_{*} \Delta_{123}$, so that if $\left\{\pi_{X}^{i}\right\}$ is self-dual, then $\left\{\pi_{X}^{i}\right\}$ is multiplicative if and only if

$$
\left(\pi_{X}^{k} \otimes \pi_{X}^{i} \otimes \pi_{X}^{j}\right)_{*} \Delta_{123}=0 \in \mathrm{CH}^{2 d}(X \times X \times X) \quad \text { whenever } i+j+k \neq 4 d,
$$

that is, if and only if the small diagonal $\Delta_{123}$ sits in $\mathrm{CH}^{2 d}\left(X^{3}\right)_{0}$ for the product Chow-Künneth decomposition on $X^{3}$; see also [12, Proposition 8.4]. In this section, we study the stability of having a (self-dual) multiplicative Chow-Künneth decomposition under product, projective bundle, blow-up, and contraction under a generically finite morphism.

3.1. Product varieties. We recall the following easy but crucial property of multiplicative Chow-Künneth decomposition:

Proposition 3.2 [12, Theorem 8.6]. Let $X$ and $Y$ be smooth projective varieties, each endowed with a multiplicative Chow-Künneth decomposition $\left\{\pi_{X}^{i}\right\}$ and $\left\{\pi_{Y}^{i}\right\}$, respectively. Then the product Chow-Künneth decomposition $\left\{\pi_{X \times Y}^{i}\right\}$ defined in (3) is multiplicative. Furthermore, if all the Chern classes of $X$ and $Y$ are in the graded-0 part, then so are the Chern classes of $X \times Y$.

Proof. We include a proof for the sake of completeness. Let $p_{X}: X \times Y \times X \times$ $Y \times X \times Y \rightarrow X \times X \times X$ be the projection on the first, third and fifth factors, and let $p_{Y}$ denote the projection on the second, fourth and sixth factors. Writing $\Delta_{123}^{X}$ for the small diagonal of $X$ and similarly for $Y$ and $X \times Y$, we have the identity

$$
\Delta_{123}^{X \times Y}=p_{X}^{*} \Delta_{123}^{X} \cdot p_{Y}^{*} \Delta_{123}^{Y} .
$$


We immediately deduce that

$$
\begin{aligned}
& \pi_{X \times Y}^{a} \circ \Delta_{123}^{X \times Y} \circ\left(\pi_{X \times Y}^{b} \otimes \pi_{X \times Y}^{c}\right) \\
& \quad=\sum_{\substack{i+i^{\prime}=a \\
j+j^{\prime}=b \\
k+k^{\prime}=c}} p_{X}^{*}\left[\pi_{X}^{i} \circ \Delta_{123}^{X} \circ\left(\pi_{X}^{j} \otimes \pi_{X}^{k}\right)\right] \cdot p_{Y}^{*}\left[\pi_{Y}^{i^{\prime}} \circ \Delta_{123}^{Y} \circ\left(\pi_{Y}^{j^{\prime}} \otimes \pi_{Y}^{k^{\prime}}\right)\right] .
\end{aligned}
$$

By definition of multiplicativity, the cycles $\pi_{X}^{i} \circ \Delta_{123}^{X} \circ\left(\pi_{X}^{j} \otimes \pi_{X}^{k}\right)$ and $\pi_{Y}^{i^{\prime}} \circ$ $\Delta_{123}^{Y} \circ\left(\pi_{Y}^{j^{\prime}} \otimes \pi_{Y}^{k^{\prime}}\right)$ are both nonzero only if $i=j+k$ and $i^{\prime}=j^{\prime}+k^{\prime}$. Therefore,

$$
\pi_{X \times Y}^{a} \circ \Delta_{123}^{X \times Y} \circ\left(\pi_{X \times Y}^{b} \otimes \pi_{X \times Y}^{c}\right)=0 \quad \text { if } a \neq b+c .
$$

This exactly means that the product Chow-Künneth decomposition on $X \times Y$ is multiplicative.

For the statement concerning the Chern classes, we first note that

$$
p_{1}^{*} \mathrm{CH}^{p}(X)_{s} \subset \mathrm{CH}^{p}(X \times Y)_{s} \quad \text { and } \quad p_{2}^{*} \mathrm{CH}^{q}(Y)_{r} \subset \mathrm{CH}^{q}(X \times Y)_{r},
$$

where $p_{1}$ and $p_{2}$ are the two projections of $X \times Y$ onto the two factors; see [12, Proposition 8.7]. It follows from the isomorphism

$$
\mathscr{T}_{X \times Y} \cong p_{1}^{*} \mathscr{T}_{X} \oplus p_{2}^{*} \mathscr{T}_{Y}
$$

that $c(X \times Y)=p_{1}^{*} c(X) \cdot p_{2}^{*} c(Y) \in \mathrm{CH}^{*}(X \times Y)_{0}$ as long as $c(X) \in \mathrm{CH}^{*}(X)_{0}$ and $c(Y) \in \mathrm{CH}^{*}(Y)_{0}$.

3.2. Projective bundles. The notations are those of Paragraph $2.2 ; \pi$ : $\mathrm{P}(\mathscr{E}) \rightarrow X$ denotes the geometric projectivization of the vector bundle $\mathscr{E}$ on $X$.

PROPOSITION 3.3. Let $X$ be a smooth projective variety of dimension d endowed with a self-dual multiplicative Chow-Künneth decomposition $\left\{\pi_{X}^{i}\right\}$ and let $\mathscr{E}$ be a vector bundle on $X$ of rank $r+1$. Assume that the Chern classes $c_{p}(\mathscr{E})$ sit in $\mathrm{CH}^{p}(X)_{0}$ for all $p \geqslant 0$. Then the self-dual Chow-Künneth decomposition $\left\{\pi_{\mathrm{P}(\mathcal{E})}^{i}\right\}$ of $\mathbb{P}(\mathscr{E})$ defined in (8) is multiplicative. If all the Chern classes of $X$ are in the graded-0 part, then so are the Chern classes of $\mathbb{P}(\mathscr{E})$. Moreover,

$$
\mathrm{CH}^{p}(\mathbb{P}(\mathscr{E}))_{s}:=\left(\pi_{\mathbb{P}(\mathscr{E})}^{2 p-s}\right)_{*} \mathrm{CH}^{p}(\mathbb{P}(\mathscr{E}))=\bigoplus_{l=0}^{r} \xi^{l} \cdot \pi^{*} \mathrm{CH}^{p-l}(X)_{s},
$$

where $\mathrm{CH}^{p}(X)_{s}:=\left(\pi_{X}^{2 p-s}\right)_{*} \mathrm{CH}^{p}(X)$. Under the above Chow-Künneth decompositions, the natural morphism $\pi: \mathbb{P}(\mathscr{E}) \rightarrow X$ is of pure grade 0 . 
Proof. It is clear that the Chow-Künneth decomposition $\left\{p_{\mathrm{P}(\mathscr{E})}^{i}\right\}$ defined in (6) induces the decomposition (16) of the Chow groups of $\mathbb{P}(\mathscr{E})$. We first show that the self-dual Chow-Künneth decomposition $\left\{\pi_{\mathrm{P}(\mathscr{E})}^{i}\right\}$ in Proposition 2.8 induces the same decomposition (16). With notations as in the proof of Proposition 2.8, it is enough to check that the correspondence $\eta:=\sigma \circ{ }^{t} \Phi \circ \Phi-$ id preserves the grading on $\mathrm{CH}^{*}\left(\bigoplus_{l=0}^{r} \mathfrak{h}(X)(l)\right)$ induced by the Chow-Künneth decomposition $\left\{\pi_{X}^{i}\right\}$. By definition of $\Phi$, it suffices to check (in the general situation where $X$ is any smooth projective variety with a self-dual multiplicative Chow-Künneth decomposition $\left.\left\{\pi_{X}^{i}\right\}\right)$ that if $\alpha$ is a cycle in $\mathrm{CH}^{*}(X)_{s}$, then $\pi_{*}\left(\xi^{i} \cdot \pi^{*} \alpha\right)$ sits in $\mathrm{CH}^{*}(X)_{s}$. But then, the projection formula gives $\pi_{*}\left(\xi^{i} \cdot \pi^{*} \alpha\right)=\alpha \cdot \pi_{*}\left(\xi^{i}\right)$. By multiplicativity of the Chow-Künneth decomposition $\left\{\pi_{X}^{i}\right\}$, we will be done if $\pi_{*}\left(\xi^{i}\right)$ sits in $\mathrm{CH}^{*}(X)_{0}$. The Chow ring of $\mathbb{P}(\mathscr{E})$ is given by

$$
\begin{aligned}
& \mathrm{CH}^{*}(\mathbb{P}(\mathscr{E}))=\mathrm{CH}^{*}(X)[\xi], \quad \text { where } \\
& \quad \xi^{r+1}+\pi^{*} c_{1}(\mathscr{E}) \xi^{r}+\cdots+\pi^{*} c_{r}(\mathscr{E}) \xi+\pi^{*} c_{r+1}(\mathscr{E})=0,
\end{aligned}
$$

from which it follows that $\pi_{*} \xi^{i}$ is a polynomial in the Chern classes of $\mathscr{E}$. By assumption the Chern classes of $\mathscr{E}$ belong to $\mathrm{CH}^{*}(X)_{0}$, and we conclude that $\pi_{*}\left(\xi^{i}\right)$ sits in $\mathrm{CH}^{*}(X)_{0}$ by multiplicativity of $\left\{\pi_{X}^{i}\right\}$.

The triple product $\mathbb{P}(\mathscr{E}) \times \mathbb{P}(\mathscr{E}) \times \mathbb{P}(\mathscr{E})$ is obtained by taking successively the projectivization of three vector bundles on $X \times X \times X$. Therefore, we may repeat the above argument, and we see that the decomposition of the Chow groups of $\mathbb{P}(\mathscr{E}) \times \mathbb{P}(\mathscr{E}) \times \mathbb{P}(\mathscr{E})$ is the same with respect to the following two ChowKünneth decompositions: (1) the Chow-Künneth decomposition $\left\{P^{i}\right\}$ obtained as the 3-fold product of $\left\{p_{\mathrm{P}(\mathscr{E})}^{i}\right\}$, and (2) the Chow-Künneth decomposition $\left\{\Pi^{i}\right\}$ obtained as the 3 -fold product of $\left\{\pi_{\mathrm{P}(\mathscr{E})}^{i}\right\}$.

In [12, Proposition 13.1], we proved under the assumptions of the proposition that the Chow-Künneth decomposition $\left\{p_{\mathrm{P}(\mathscr{E})}^{i}\right\}$ is multiplicative. By (15), this means that the small diagonal $\Delta_{123}^{\mathrm{P}(\mathscr{E})}$ belongs to $\left(P^{4(d+r)}\right)_{*} \mathrm{CH}_{d+r}(\mathbb{P}(\mathscr{E}) \times$ $\mathrm{P}(\mathscr{E}) \times \mathbb{P}(\mathscr{E}))$. Thus we obtain from the above that $\Delta_{123}^{\mathrm{P}(\mathscr{E})}$ belongs to $\left(\Pi^{4(d+r)}\right)_{*} \mathrm{CH}_{d+r}(\mathrm{P}(\mathscr{E}) \times \mathbb{P}(\mathscr{E}) \times \mathbb{P}(\mathscr{E}))$. Therefore, by (15), the Chow-Künneth decomposition $\left\{\pi_{\mathrm{P}(\mathscr{E})}^{i}\right\}$ is multiplicative.

For the statement concerning the Chern classes, we only need to show that the Chern classes of the relative tangent bundle $\widetilde{T}_{\mathbb{P}(\mathscr{E}) / X}$ are in the graded- 0 part. But this follows immediately from the short exact sequence

$$
0 \longrightarrow \mathcal{O}_{\mathbb{P}(\mathscr{E})} \longrightarrow \pi^{*} \mathscr{E} \otimes \mathcal{O}(1) \longrightarrow \mathscr{T}_{\mathbb{P}(\mathscr{E}) / X} \longrightarrow 0
$$

by taking Chern classes of the sheaves involved.

As in Remark 2.9, we take $Y=X$ and let

$$
\pi \times \text { id }: \mathbb{P}(\mathscr{E}) \times X \rightarrow X \times X
$$


be the product morphism. Then the fact that $\Delta_{X}$ belongs to $\mathrm{CH}^{d_{X}}(X \times X)_{0}$ implies

$$
\Gamma_{\pi}=(\pi \times \mathrm{id})^{*} \Delta_{X} \in \mathrm{CH}^{d_{X}}(\mathbb{P}(\mathscr{E}) \times X)_{0} .
$$

Hence $\pi$ is of pure grade 0 .

3.3. Blow-ups. We take on the notations from paragraph 2.3; the embedding $i: Y \hookrightarrow X$ is a smooth closed subvariety of codimension $r+1$ and $\tilde{X}:=\mathrm{Bl}_{Y}(X)$ is the blow-up of $X$ along $Y$, and we have the blow-up diagram (9)

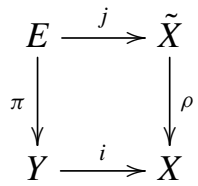

where $E \cong \mathbb{P}\left(\mathscr{N}_{Y / X}\right)$ is the exceptional divisor. Recall from the blow-up formula (10) that the Chow groups of $\tilde{X}$ can be described explicitly as

$$
\begin{gathered}
\Phi_{*}: \mathrm{CH}^{p}(X) \oplus\left(\bigoplus_{l=1}^{r} \mathrm{CH}^{p-l}(Y)\right) \stackrel{\cong}{\longrightarrow} \mathrm{CH}^{p}(\tilde{X}), \\
\left(\alpha, \beta_{1}, \ldots, \beta_{r}\right) \mapsto \rho^{*} \alpha+j_{*}\left(\sum_{l=1}^{r} \xi^{l-1} \cdot \pi^{*} \beta_{l}\right),
\end{gathered}
$$

where $\xi \in \mathrm{CH}^{1}(E)$ is the Chern class of the relative $\mathcal{O}(1)$-bundle.

Proposition 3.4. Assume that both $X$ and $Y$ admit self-dual multiplicative Chow-Künneth decompositions $\left\{\pi_{X}^{i}\right\}$ and $\left\{\pi_{Y}^{i}\right\}$, respectively, such that:

(i) the Chern classes of the normal bundle $\mathscr{N}_{Y / X}$ sit in $\mathrm{CH}^{*}(Y)_{0}$;

(ii) the morphism $i: Y \rightarrow X$ is of pure grade 0 .

Then the self-dual Chow-Künneth decomposition (13) of $\tilde{X}$ is multiplicative. Moreover,

$$
\mathrm{CH}^{p}(\tilde{X})_{s}:=\left(\pi_{\tilde{X}}^{2 p-s}\right)_{*} \mathrm{CH}^{p}(\tilde{X})=\rho^{*} \mathrm{CH}^{p}(X)_{s} \oplus\left(\bigoplus_{l=1}^{r} j_{*}\left(\xi^{l-1} \cdot \pi^{*} \mathrm{CH}^{p-l}(Y)_{s}\right)\right),
$$

where $\mathrm{CH}^{p}(X)_{s}=\left(\pi_{X}^{2 p-s}\right)_{*} \mathrm{CH}^{p}(X)$ and $\mathrm{CH}^{p}(Y)_{s}=\left(\pi_{Y}^{2 p-s}\right)_{*} \mathrm{CH}^{p}(Y)$ and $\xi:=$ $c_{1}\left(\left.\mathcal{O}_{\tilde{X}}(-E)\right|_{E}\right)$. Furthermore, if the Chern classes of $X$ are in the graded-0 part, then so are the Chern classes of $\tilde{X}$. The natural blow-up morphism $\rho$ is of pure grade 0 . 
Proof. The proof is similar to that of [12, Proposition 13.2], and consequently we only sketch the main steps. The difference with [12, Proposition 13.2] is that we have removed the injectivity assumption on $i_{*}$. For that purpose, an explicit computation of the small diagonal is carried out in Lemma 3.5. Note that the assumption (ii) implies that $i^{*}$ and $i_{*}$ are compatible with the gradings on the Chow groups, namely

$$
i_{*} \mathrm{CH}^{p}(Y)_{s} \subseteq \mathrm{CH}^{p+r+1}(X)_{s}, \quad i^{*} \mathrm{CH}^{p}(X)_{s} \subseteq \mathrm{CH}^{p}(Y)_{s} .
$$

In addition to that, by (i) of Proposition 2.6, the push-forward and pull-back via $i \times \mathrm{id}_{Z}: Y \times Z \rightarrow X \times Z$ also respect the gradings on Chow ring, for all smooth projective variety $Z$ endowed with a multiplicative Chow-Künneth decomposition.

Step 1. Without the condition of being self-dual, by [12, Proposition 13.2], we can construct a Chow-Künneth decomposition $\left\{p_{\tilde{X}}^{i}\right\}$ of $\tilde{X}$ such that the induced grading on the Chow ring is the same as the one given in equation (17). Furthermore, the homomorphisms $j_{*}$ and $j^{*}$ are compatible with the gradings, where $\mathrm{CH}^{*}(E)_{s}$ is given by (16) which is induced by a self-dual multiplicative Chow-Künneth decomposition on $E$. More generally, if $Z$ is a smooth projective variety with a multiplicative Chow-Künneth decomposition, then

$$
\begin{gathered}
\left(j \times \mathrm{id}_{Z}\right)_{*}: \mathrm{CH}^{p}(E \times Z) \rightarrow \mathrm{CH}^{p+1}(\tilde{X} \times Z) \text { and } \\
\left(j \times \mathrm{id}_{Z}\right)^{*}: \mathrm{CH}^{p}(\tilde{X} \times Z) \rightarrow \mathrm{CH}^{p}(E \times Z)
\end{gathered}
$$

respect the gradings. Indeed, the actions of $\left(i \times \mathrm{id}_{Z}\right)_{*}$ and $\left(i \times \mathrm{id}_{Z}\right)^{*}$ respect the gradings of $\mathrm{CH}^{*}(Y \times Z)$ and $\mathrm{CH}^{*}(X \times Z)$ and hence the argument for the compatibility of $j_{*}$ and $j^{*}$ with the grading of Chow groups (as in the proof of [12, Proposition 13.2]) applies to show the compatibility of $\left(j \times \mathrm{id}_{Z}\right)_{*}$ and $\left(j \times \mathrm{id}_{Z}\right)^{*}$ with the gradings. We apply this successively to

$$
E \times E \times E \longrightarrow E \times E \times \tilde{X} \longrightarrow E \times \tilde{X} \times \tilde{X} \longrightarrow \tilde{X} \times \tilde{X} \times \tilde{X}
$$

and show that the pull-back and push-forward of $j^{\times 3}: E^{3} \rightarrow \tilde{X}^{3}$ respect the grading on the Chow groups. This was implicitly used in the proof of [12, Proposition 13.2] but the assumption there (compatibility of $i^{*}$ and $i_{*}$ with the grading on Chow groups) is insufficient to deduce it.

Step 2. The self-product $\tilde{X} \times \tilde{X} \times \tilde{X}$ has two natural Chow-Künneth decompositions. The first one is the 3 -fold product of $\left\{p_{\tilde{X}}^{i}\right\}$. The second one is obtained when $\tilde{X}^{3}$ is viewed as the successive blow-up of $X^{3}$ along $Y \times X \times X, \tilde{X} \times Y \times X$, and $\tilde{X} \times \tilde{X} \times Y$, where $X^{3}$ is given the product ChowKünneth decomposition. Then it turns out that the above two Chow-Künneth 
decompositions are the same; see the proof of [12, Proposition 13.2]. As a consequence we have

$$
\left(\rho^{\times 3}\right)^{*} \mathrm{CH}\left(X^{3}\right)_{0}+\left(j^{\times 3}\right)_{*}\left(\xi_{1}^{i_{1}} \xi_{2}^{i_{2}} \xi_{3}^{i_{3}} \cdot\left(\pi^{\times 3}\right)^{*} \mathrm{CH}^{*}\left(Y^{3}\right)_{0}\right) \subseteq \mathrm{CH}^{*}\left(\tilde{X}^{3}\right)_{0} .
$$

Here the notation is as follows. If $f: Z \rightarrow Z^{\prime}$ is a morphism, then $f^{\times 3}: Z^{3} \rightarrow Z^{\prime 3}$ is the 3-fold self-product of $f$; the class $\xi_{i} \in \mathrm{CH}^{1}\left(E^{3}\right)$ is the pull-back of $\xi$ via the projection onto the $i$ th factor, $i=1,2,3$.

Step 3. The small diagonal $\Delta_{\tilde{X}}^{123} \in \mathrm{CH}^{2 d}\left(\tilde{X}^{3}\right)$ is contained in the graded-0 part. This was proved in [12, Proposition 13.2] under the assumption that $i_{*}$ is injective. Without assuming that $i_{*}$ is injective, this follows immediately from Lemma 3.5 and equation (18).

Step 4 . The above three steps can be carried out if we replace $p_{\tilde{X}}^{i}$ by $\pi_{\tilde{X}}^{i}$. We have seen that the small diagonal $\Delta_{123}^{\tilde{X}}$ belongs to $\left(p_{\tilde{X} \times \tilde{X} \times \tilde{X}}^{4 d}\right)_{*} \mathrm{CH}_{d}(\tilde{X} \times \tilde{X} \times \tilde{X})$, where $p_{\tilde{X} \times \tilde{X} \times \tilde{X}}^{4 d}:=\sum_{i+j+k=4 d} p_{\tilde{X}}^{i} \otimes p_{\tilde{X}}^{j} \otimes p_{\tilde{X}}^{k}$ and $p_{\tilde{X}}^{i}$ is the idempotent defined in (11). In order to prove the proposition, it suffices as in the proof of Proposition 3.3 to show that the Chow-Künneth decomposition $\left\{\pi_{\tilde{X}}^{i}\right\}$ of (13) satisfies

$$
\begin{aligned}
\left(\pi_{\tilde{X}}^{i}\right)_{*} \mathrm{CH}^{p}(\tilde{X}) & =\left(p_{\tilde{X}}^{i}\right)_{*} \mathrm{CH}^{p}(\tilde{X}) \\
& =\rho^{*} \mathrm{CH}^{p}(X)_{2 i-s} \oplus\left(\bigoplus_{l=0}^{r-1} j_{*}\left(\xi^{l} \cdot \pi^{*} \mathrm{CH}^{p-l-1}(Y)_{2 i-s}\right)\right) .
\end{aligned}
$$

For this it is enough to show that $\eta:=\sigma \circ D \circ{ }^{t} \Phi \circ \Phi-\mathrm{id}$ preserves the grading on $\mathrm{CH}^{*}\left(\mathfrak{h}(X) \oplus \bigoplus_{l=1}^{r} \mathfrak{h}(Y)(l)\right)$. It is in fact enough to show that ${ }^{t} \Phi \circ \Phi$ preserves the grading. By (12), we only need to show that $\rho_{*} \rho^{*} \mathrm{CH}^{*}(X)_{s} \subseteq \mathrm{CH}^{*}(X)_{s}$ and that $\pi_{*} \circ h^{l} \circ \pi^{*} \mathrm{CH}^{*}(Y)_{s} \subseteq \mathrm{CH}^{*}(Y)_{s}$ for all $l \geqslant 0$ and all integers $s$. The first inclusion is obvious because $\rho_{*} \rho^{*}$ is the identity on $\mathrm{CH}^{*}(X)$, and the second inclusion was already established in the proof of Proposition 3.3.

The statement concerning the Chern classes follows from the short exact sequence in Lemma 4.5, which again follows from a Chern class computation as before.

Now we take $X^{\prime}=X$ in Remark 2.11 and note that

$$
\Gamma_{\rho}=(\rho \times \mathrm{id})^{*} \Delta_{X} .
$$

This implies that $\rho$ is of pure grade 0 since $\Delta_{X} \in \mathrm{CH}^{d_{X}}(X \times X)_{0}$.

LEMMA 3.5. Let $\rho^{\times l}: \tilde{X}^{l} \rightarrow X^{l}$ be the l-fold self-product of $\rho$ and let

$$
j_{/ Y}^{\times l}: E_{Y}^{\times l}=E \times_{Y} E \times_{Y} \cdots \times_{Y} E \rightarrow \tilde{X}^{l}
$$


be the closed immersion induced by j. Let $\mathscr{N}_{l}$ be the sheaf on $E_{Y}^{\times l}$ that is obtained as the pull-back of $\mathscr{N}_{Y / X}$ from $Y$ and let $\xi_{k} \in \mathrm{CH}^{1}\left(E_{Y}^{\times l}\right)$ be the pull-back of $\xi \in \mathrm{CH}^{1}(E)$ from the $k$ th factor, $1 \leqslant k \leqslant l$. Then the following equality holds in $\mathrm{CH}_{d}\left(\tilde{X}^{2}\right)$

$$
(\rho \times \rho)^{*} \Delta_{X}=\Delta_{\tilde{X}}+\left(j \times \times_{Y} j\right)_{*}\left[\frac{c\left(\mathscr{N}_{2}\right)}{\left(1-\xi_{1}\right)\left(1-\xi_{2}\right)}\right]_{d},
$$

where $[-]_{d}$ takes the dimension d component. More generally,

$$
\left(\rho^{\times l}\right)^{*} \Delta_{X}^{(l)}=\Delta_{\tilde{X}}^{(l)}+\left(j_{/ Y}^{\times l}\right)_{*} P\left(\xi_{k}, c\left(\mathscr{N}_{l}\right)\right),
$$

where $P(-)$ is a polynomial, $\Delta_{X}^{(l)}=\{(x, x, \ldots, x)\} \subset X^{l}$ is the small diagonal.

Proof. Let $\Delta_{i j}\left(E_{/ Y}\right) \in \mathrm{CH}^{r}\left(E_{/ Y}^{\times l}\right)$ be the relative (over $Y$ ) bigger diagonal given by $\left\{\left(x_{1}, \ldots, x_{l}\right): x_{i}=x_{j}\right\}$. We claim that $\Delta_{i j}(E / Y)$ is a polynomial of $\xi_{i}$, $\xi_{j}$ and $c\left(\mathscr{N}_{l}\right)$. To prove this claim, it suffices to show that in the case $l=2$. In this case we have the natural homomorphisms of sheaves

$$
\mathcal{O}\left(-\xi_{1}\right) \rightarrow \mathscr{N}_{2} \text { and } \mathcal{O}\left(-\xi_{2}\right) \rightarrow \mathscr{N}_{2}
$$

and they give rise to a section $\alpha$ of $\left(\mathscr{N}_{2} / \mathcal{O}\left(-\xi_{1}\right)\right) \otimes \mathcal{O}\left(\xi_{2}\right)$. The cycle $\Delta_{12}(E / Y)$ is the vanishing locus of $\alpha$ and hence its class is the top Chern class of the above sheaf. The claim follows immediately. Hence it suffices to show that

$$
\left(\rho^{\times l}\right)^{*} \Delta_{X}^{(l)}=\Delta_{\tilde{X}}^{(l)}+\left(j_{/ Y}^{\times l}\right)_{*} P\left(\Delta_{i j}\left(E_{/ Y}\right), \xi_{k}, c\left(\mathscr{N}_{l}\right)\right),
$$

for some polynomial $P$.

Note that $\tilde{X} \times \tilde{X}$ can be viewed as a successive blow-up of $X \times X$ as follows

$$
\tilde{X} \times \tilde{X} \stackrel{\operatorname{id}_{\tilde{X}} \times \rho}{\longrightarrow} \tilde{X} \times X \stackrel{\rho \times \operatorname{id}_{X}}{\longrightarrow} X \times X .
$$

For each blow-up, we use [6, Theorem 6.7] (Blow-up Formula) and easily get

$$
\left(\rho \times \operatorname{id}_{X}\right)^{*} \Delta_{X}=\Gamma_{\rho}
$$

and

$$
\begin{aligned}
& \left(\operatorname{id}_{\tilde{X}} \times \rho\right)^{*} \Gamma_{\rho} \\
& \quad=\Delta_{\tilde{X}}+\left(\operatorname{id}_{\tilde{X}} \times j\right)_{*}\left[\left(p_{\tilde{X} \times E, 2}\right)^{*} c\left(\frac{\pi^{*} \mathscr{N}_{Y / X}}{\mathcal{O}_{E}(-1)}\right) \cap\left(\operatorname{id}_{X} \times \pi\right)^{*} s(E, \tilde{X})\right]_{d} \\
& \quad=\Delta_{\tilde{X}}+\left(j \times_{Y} j\right)_{*}\left[\frac{c\left(\mathscr{N}_{l}\right)}{\left(1-\xi_{1}\right)\left(1-\xi_{2}\right)}\right]_{d}
\end{aligned}
$$


The general case follows by induction on $l$. The induction step is established by observing that

$$
\left(\rho^{\times(l+1)}\right)^{*} \Delta_{X}^{(l+1)}=\left(\left(\rho^{\times l}\right)^{*} \Delta_{X}^{(l)} \times \tilde{X}\right) \cdot\left(\tilde{X}^{l-1} \times(\rho \times \rho)^{*} \Delta_{X}\right)
$$

and by applying [6, Theorem 6.3] (Excess Intersection Formula) to the following square

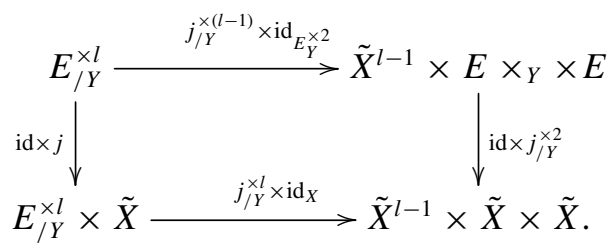

REMARK 3.6. Under the assumptions of Proposition 3.4, all the varieties involved in the blow-up diagram (9), namely $E, Y, X$ and $\tilde{X}$, are given a selfdual multiplicative Chow-Künneth decomposition such that all the morphisms involved in that diagram, namely $i, \pi, j$ and $\rho$, are of pure grade 0 . This fact will be needed in the proof of Lemma 6.2 to show that a certain set of closed subvarieties is admissible.

By Proposition 3.4, it only remains to show that the closed immersion $j$ is of pure grade 0 . In what follows, we will use the notations from the proof of Proposition 2.10. We will also borrow the notations from the proof of Proposition 2.8 and we add a " " to each notation to avoid possible confusion. Note that $E \cong$ $\mathbb{P}\left(\mathscr{N}_{Y / X}\right)$ is naturally a projective bundle over $Y$. Let

$$
\Phi^{\prime}=\left(\bigoplus_{l=0}^{r-1} h^{l} \circ{ }^{t} \Gamma_{\pi}\right) \oplus \gamma^{\prime} \circ{ }^{t} \Gamma_{\pi}:\left(\bigoplus_{l=0}^{r-1} \mathfrak{h}(Y)(l)\right) \oplus \mathfrak{h}(Y)(r) \longrightarrow \mathfrak{h}(E)
$$

be the isomorphism defined in Section 2.2 (note that this was denoted $\Phi$ there). Then we have a commutative diagram

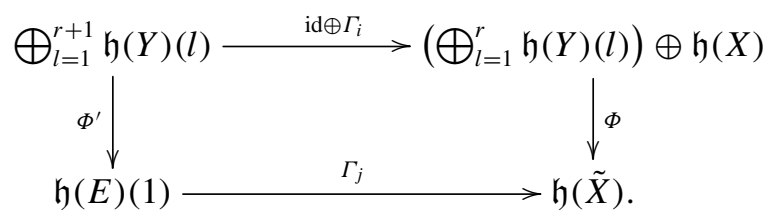

The only nontrivial part of the above commutativity is

$$
\left.\Phi\right|_{\mathfrak{h}(X)} \circ \Gamma_{i}=\Gamma_{j} \circ\left(\gamma^{\prime} \circ{ }^{t} \Gamma_{\pi}\right) .
$$


Note that $\left.\Phi\right|_{\mathfrak{h}(X)}={ }^{t} \Gamma_{\rho}$, and one easily sees that the above identity is simply the 'key formula' of [6, Proposition 6.7(a)] stated at the level of correspondences. It is clear that the commutativity holds only when $\Phi^{\prime}$ is taken to be the modified projective bundle formula isomorphism, instead of the usual one; see Section 2.2. Let $\sigma^{\prime}, \eta^{\prime}$ and $\Psi^{\prime}=\Phi^{\prime} \circ\left(\mathrm{id}+\eta^{\prime}\right)^{-1 / 2}$ be the corresponding $\sigma, \eta$ and $\Psi$ of the proof of Proposition 2.8 for the projective bundle $\pi: E \rightarrow Y$. By comparing (7) and (12), we get

$$
\left.\left.\Gamma_{j} \circ \Phi^{\prime}\right|_{\oplus_{l=1}^{r} \mathfrak{h}(Y)(l)} \circ \eta^{\prime}\right|_{\oplus_{l=1}^{r} \mathfrak{h}(Y)(l)}=\left.\left.\Phi\right|_{\oplus_{l=1}^{r} \mathfrak{h}(Y)(l)} \circ \eta\right|_{\oplus_{l=1}^{r} \mathfrak{h}(Y)(l)} .
$$

As a result, we have

$$
\Gamma_{j} \circ \Psi^{\prime}=\Psi \circ\left(\mathrm{id} \oplus \Gamma_{i}\right), \quad \text { on } \bigoplus_{l=1}^{r} \mathfrak{h}(Y)(l) .
$$

Note that $\left.\eta^{\prime}\right|_{\mathfrak{h}(Y)(r+1)}=\cdot c_{r}\left(\mathscr{N}_{Y / X}\right): \mathfrak{h}(Y)(r+1) \rightarrow \mathfrak{h}(Y)(1)$ and $\left.\eta^{\prime}\right|_{\mathfrak{h}(Y)(1)}=0$, and hence $\eta^{\prime o l}=0$ on $\mathfrak{h}(Y)(r+1)$ for all $l \geqslant 2$. As a consequence

$$
\left.\Gamma_{j} \circ \Psi^{\prime}\right|_{\mathfrak{h}(Y)(r+1)}=\left.\Gamma_{j} \circ \Phi^{\prime} \circ\left(1-\frac{1}{2} \eta^{\prime}\right)\right|_{\mathfrak{h}(Y)(r+1)} .
$$

Meanwhile, since $\left.\eta\right|_{\mathfrak{h}(X)}=0$, one easily shows that

$$
\left.\Psi \circ\left(\mathrm{id} \oplus \Gamma_{i}\right)\right|_{\mathfrak{h}(Y)(r+1)}=\left.\Phi \circ(1+\eta)^{-1 / 2}\right|_{\mathfrak{h}(X)} \circ \Gamma_{i}=\Phi \circ \Gamma_{i} .
$$

It follows that

$$
\begin{aligned}
\Gamma_{j} \circ \Psi^{\prime} & =\Psi \circ\left(\operatorname{id} \oplus \Gamma_{i}\right)-\left.\frac{1}{2}\left(\Gamma_{j} \circ \Phi^{\prime}\right)\right|_{\mathfrak{h}(Y)(1)} \circ\left(\cdot c_{r}\left(\mathscr{N}_{Y / X}\right)\right) \\
& =\Psi \circ\left(\operatorname{id} \oplus \Gamma_{i}\right)-\left.\frac{1}{2} \Phi\right|_{\mathfrak{h}(Y)(1)} \circ\left(\cdot c_{r}\left(\mathscr{N}_{Y / X}\right)\right) \\
& =\Psi \circ\left(\operatorname{id} \oplus \Gamma_{i}\right)-\left.\frac{1}{2} \Psi\right|_{\mathfrak{h}(Y)(1)} \circ\left(\cdot c_{r}\left(\mathscr{N}_{Y / X}\right)\right) .
\end{aligned}
$$

Here the last equality uses $\left.\Psi\right|_{\mathfrak{h}(Y)(1)}=\left.\Phi\right|_{\mathfrak{h}(Y)(1)}$, which follows from the fact that $\left.\eta\right|_{\mathfrak{h}(Y)(1)}=0$. Hence we get

$$
\Gamma_{j}=\Psi \circ\left(\operatorname{id} \oplus \Gamma_{i}\right) \circ\left(\Psi^{\prime}\right)^{-1}-\left.\frac{1}{2} \Psi\right|_{\mathfrak{h}(Y)(1)} \circ\left(\cdot c_{r}\left(\mathscr{N}_{Y / X}\right)\right) \circ\left(\Psi^{\prime}\right)^{-1} .
$$

This equality implies that $\Gamma_{j}$ is of pure grade 0 since all the morphisms appearing on the right-hand side are of pure grade 0 .

3.4. Generically finite quotients. The following proposition shows that the Chow-Künneth decomposition constructed in Proposition 2.12 is multiplicative. 
Proposition 3.7. Let $p: X \rightarrow Y$ be a generically finite morphism between smooth projective varieties. Assume that $X$ is endowed with a self-dual multiplicative Chow-Künneth decomposition $\left\{\pi_{X}^{i}\right\}$ and that

$$
{ }^{t} \Gamma_{p} \circ \Gamma_{p} \in \mathrm{CH}_{d}(X \times X)_{0}, \quad d=\operatorname{dim} X .
$$

Then the Chow-Künneth decomposition of $Y$, as given in Proposition 2.12, is also multiplicative.

Proof. Let $N$ be the degree of the morphism $p$. We first note that

$$
\Delta_{123}^{Y}=\frac{1}{N}(p \times p \times p)_{*} \Delta_{123}^{X}=\frac{1}{N} \Gamma_{p} \circ \Delta_{123}^{X} \circ\left({ }^{t} \Gamma_{p} \otimes{ }^{t} \Gamma_{p}\right)
$$

and that

$$
\begin{aligned}
\left({ }^{t} \Gamma_{p} \otimes{ }^{t} \Gamma_{p}\right) \circ\left(\pi_{Y}^{i} \otimes \pi_{Y}^{j}\right) & =\frac{1}{N^{2}}\left({ }^{t} \Gamma_{p} \circ \Gamma_{p} \circ \pi_{X}^{i} \circ{ }^{t} \Gamma_{p}\right) \otimes\left({ }^{t} \Gamma_{p} \circ \Gamma_{p} \circ \pi_{X}^{j} \circ{ }^{t} \Gamma_{p}\right) \\
& =\frac{1}{N^{2}}\left(\pi_{X}^{i} \circ{ }^{t} \Gamma_{p} \circ \Gamma_{p} \circ{ }^{t} \Gamma_{p}\right) \otimes\left(\pi_{X}^{i} \circ{ }^{t} \Gamma_{p} \circ \Gamma_{p} \circ{ }^{t} \Gamma_{p}\right) \\
& =\left(\pi_{X}^{i} \circ{ }^{t} \Gamma_{p}\right) \otimes\left(\pi_{X}^{j} \circ{ }^{t} \Gamma_{p}\right) \\
& =\left(\pi_{X}^{i} \otimes \pi_{X}^{j}\right) \circ\left({ }^{t} \Gamma_{p} \otimes{ }^{t} \Gamma_{p}\right) .
\end{aligned}
$$

Hence we have

$$
\begin{aligned}
\pi_{Y}^{k} \circ \Delta_{123}^{Y} \circ\left(\pi_{Y}^{i} \otimes \pi_{Y}^{j}\right) & =\frac{1}{N^{2}} \Gamma_{p} \circ \pi_{X}^{k} \circ{ }^{t} \Gamma_{p} \circ \Gamma_{p} \circ \Delta_{123}^{X} \circ\left({ }^{t} \Gamma_{p} \otimes{ }^{t} \Gamma_{p}\right) \circ\left(\pi_{Y}^{i} \otimes \pi_{Y}^{j}\right) \\
& =\frac{1}{N^{2}} \Gamma_{p} \circ{ }^{t} \Gamma_{p} \circ \Gamma_{p} \circ \pi_{X}^{k} \circ \Delta_{123}^{X} \circ\left(\pi_{X}^{i} \otimes \pi_{X}^{j}\right) \circ\left({ }^{t} \Gamma_{p} \otimes{ }^{t} \Gamma_{p}\right) \\
& =\frac{1}{N} \Gamma_{p} \circ \pi_{X}^{k} \circ \Delta_{123}^{X} \circ\left(\pi_{X}^{i} \otimes \pi_{X}^{j}\right) \circ\left({ }^{t} \Gamma_{p} \otimes{ }^{t} \Gamma_{p}\right) .
\end{aligned}
$$

Note that in the above computation, we have used the commutativity of ${ }^{t} \Gamma_{p} \circ \Gamma_{p}$ and $\pi_{X}^{i}$ as correspondences; see Lemma 2.5. By assumption $\pi_{X}^{i}$ is multiplicative and hence

$$
\pi_{X}^{k} \circ \Delta_{123}^{X} \circ\left(\pi_{X}^{i} \otimes \pi_{X}^{j}\right)=0,
$$

for all $k \neq i+j$. It follows that

$$
\pi_{Y}^{k} \circ \Delta_{123}^{Y} \circ\left(\pi_{Y}^{i} \otimes \pi_{Y}^{j}\right)=0
$$

for all $k \neq i+j$ and this establishes the multiplicativity of $\left\{\pi_{Y}^{i}\right\}$. 
COROLLARY 3.8. Let $X$ be a smooth projective variety endowed with the action of a finite group $G$. Assume that $X$ has a $G$-invariant self-dual multiplicative Chow-Künneth decomposition $\left\{\pi_{X}^{i}\right\}$. Then the Chow-Künneth decomposition $\left\{\pi_{X / G}^{i}\right\}$ defined in (14) of the quotient variety $X / G$ is self-dual and multiplicative.

REMARK 3.9. Proposition 3.4 and Corollary 3.8 fix a couple of gaps in the proof of [12, Theorem 6]. First the proof that the Hilbert square $X^{[2]}$ can be endowed with a multiplicative Chow-Künneth decomposition that is self-dual was omitted. Second Proposition 3.4 corrects and improves [12, Proposition 13.2]: the assumption (iii) of [12, Proposition 13.2] is superfluous, while the assumption (ii) requiring $i_{*}$ and $i^{*}$ to be compatible with the gradings should be strengthened to requiring the inclusion morphism $i: Y \rightarrow X$ to be of pure grade 0 (which is a stronger condition by Proposition 2.6).

\section{Successive blow-ups}

Let $X$ be a smooth projective variety with a multiplicative Chow-Künneth decomposition. In this section, we wish to understand when a variety obtained from $X$ by successive smooth blow-ups admits a multiplicative Chow-Künneth decomposition. For that matter we provide sufficient conditions on the centers of these successive blow-ups for the resulting variety to admit a multiplicative Chow-Künneth decomposition. We consider a fairly general situation, which could prove useful in future work. We are led to formulate the following technical definition.

Definition 4.1. Let $\mathcal{S}=\left\{Y_{1}, Y_{2}, \ldots, Y_{n}\right\}$ be a finite set of closed subvarieties of $X$. The set $\mathcal{S}$ is said to be complete if the following two conditions hold:

(i) $X \in \mathcal{S}$;

(ii) if $Y_{j_{1}}, Y_{j_{2}} \in \mathcal{S}$ and the scheme-theoretic intersection $Y^{\prime}=Y_{j_{1}} \cap Y_{j_{2}}$ is nonempty, then $Y^{\prime} \in \mathcal{S}$.

The set $\mathcal{S}$ is admissible if the following conditions hold:

(i) each $Y_{j}$ is smooth and has a self-dual multiplicative Chow-Künneth decomposition;

(ii) if $Y_{j_{1}} \subset Y_{j_{2}}$ are closed subvarieties of $X$ that belong to $\mathcal{S}$ and if $i: Y_{j_{1}} \hookrightarrow Y_{j_{2}}$ denotes the embedding morphism, then 
(a) the Chern classes of the normal bundle $\mathscr{N}_{Y_{j_{1}} / Y_{j_{2}}}$ sit in $\mathrm{CH}^{*}\left(Y_{j_{1}}\right)_{0}$;

(b) the morphism $i: Y_{j_{1}} \hookrightarrow Y_{j_{2}}$ is of pure grade 0 .

REMARK 4.2. Being admissible is transitive in the following sense. If $\left\{Y_{1} \subset Y_{2}\right\}$ and $\left\{Y_{2} \subset Y_{3}\right\}$ are two admissible subsets, then $\left\{Y_{1} \subset Y_{3}\right\}$ is admissible.

The first reason for introducing admissible sets of subvarieties of $X$ lies in the following.

Proposition 4.3. Given any two elements $Y_{j_{1}}$ and $Y_{j_{2}}$ of an admissible set such that $Y_{j_{1}} \subset Y_{j_{2}}$, the blow-up of $Y_{j_{2}}$ along $Y_{j_{1}}$ has a multiplicative Chow-Künneth decomposition.

Proof. The proposition follows at once from Proposition 3.4.

Pick $Y \in \mathcal{S}$ and let $\tilde{X}$ be the blow-up of $X$ with center $Y$. Define $\operatorname{Bl}_{Y}(\mathcal{S})$ to be the subset of the set of smooth closed subvarieties of $\tilde{X}$ that consists of the strict transforms of the $Y_{j}$ for all $Y_{j} \in \mathcal{S}$ such that $Y_{j}$ is not contained in $Y$. Here is the main reason for introducing admissible sets. The following key proposition shows that admissible sets behave well after blowing-up along one of their elements, making it thus possible to avoid checking that the assumptions of Proposition 3.4 are met after each blow-up.

PROPOSITION 4.4. Let $\mathcal{S}$ be a complete admissible subset of closed subvarieties of $X$. Let $Y \in \mathcal{S}$ and let $\tilde{X}$ be the blow-up of $X$ along $Y$. Then $\mathrm{Bl}_{Y}(\mathcal{S})$ is complete and admissible.

Before proving Proposition 4.4, we state and prove three auxiliary lemmas. The notations are those of diagram (9).

First, the tangent bundles of $X$ and its blow-up $\tilde{X}$ are linked as follows.

LEMMA 4.5. With notations as in diagram (9), there is a short exact sequence

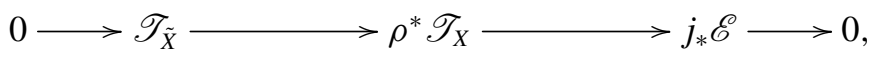

where $\mathscr{E}=\pi^{*} \mathscr{N}_{Y / X} / \mathcal{O}_{E}(-1)$.

Proof. Denote $\mathscr{Q}$ the quotient sheaf of the natural homomorphism $\mathscr{T}_{\tilde{X}} \rightarrow \rho^{*} \mathscr{T}_{X}$, so that we have a short exact sequence

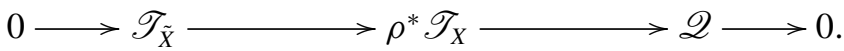


Since $\rho$ is an isomorphism away from $E$, we see that $\mathscr{Q}$ is supported on $E$. In particular, $\mathscr{Q} \otimes_{\mathcal{O}_{\tilde{X}}} \mathcal{O}_{E}=\mathscr{Q}$. Tensoring the above short exact sequence with $\mathcal{O}_{E}$ gives the exact sequence

$$
\left.\left.\mathscr{T}_{\tilde{X}}\right|_{E} \longrightarrow \rho^{*} \mathscr{T}_{X}\right|_{E} \longrightarrow \mathscr{Q} \longrightarrow 0 .
$$

This sequence fits into the following commutative diagram

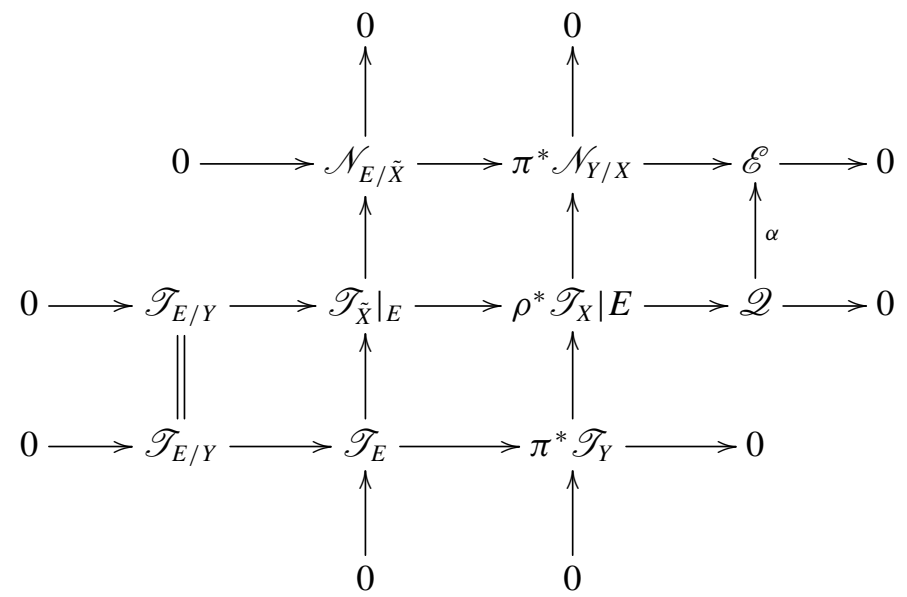

The snake lemma implies that $\alpha: \mathscr{Q} \rightarrow \mathscr{E}$ is an isomorphism and we thus have an isomorphism $\mathscr{Q} \cong j_{*} \mathscr{E}$ of torsion sheaves on $\tilde{X}$. This proves the lemma.

Secondly, the following lemma on the behavior of normal bundles under a blowup will be useful.

LEMMA 4.6. Let $X$ be a smooth projective variety and let $Y, Z \subset X$ be two smooth closed subvarieties. Assume that $Z$ is not contained in $Y$ and that the scheme-theoretic intersection $Y^{\prime}:=Y \cap Z$ is smooth. Let $\rho: \tilde{X} \rightarrow X$ be the blowup of $X$ along $Y$ and let $\tilde{Z} \subset \tilde{X}$ be the strict transform of $Z$. Let $\mathscr{N}_{Y, Z / X}$ be the locally free sheaf on $Y^{\prime}$ that is the quotient of $\left.\mathscr{T}_{X}\right|_{Y^{\prime}}$ by the subbundle generated by $\left.\mathscr{T}_{Y}\right|_{Y^{\prime}}$ and $\left.\mathscr{T}_{Z}\right|_{Y^{\prime}}$. Namely,

$$
\mathscr{N}_{Y, Z / X}:=\frac{\left.\mathscr{T}_{X}\right|_{Y^{\prime}}}{\left\langle\left.\mathscr{T}_{Y}\right|_{Y^{\prime}},\left.\mathscr{T}_{Z}\right|_{Y^{\prime}}\right\rangle} .
$$

Then there is a short exact sequence

$$
0 \longrightarrow \mathscr{N}_{\tilde{Z} / \tilde{X}} \longrightarrow \rho^{* *} \mathscr{N}_{Z / X} \longrightarrow j_{*}^{\prime} \pi^{* *} \mathscr{N}_{Y, Z / X} \longrightarrow 0
$$


Here $\rho^{\prime}: \tilde{Z} \rightarrow Z$ is the blow-up morphism; $E^{\prime} \subset \tilde{Z}$ is the exceptional divisor with $j^{\prime}: E^{\prime} \hookrightarrow \tilde{Z}$ being the closed immersion; $\pi^{\prime}: E^{\prime} \rightarrow Y^{\prime}$ is the natural projection.

In particular, if $Y \subset Z$ then $\mathscr{N}_{\tilde{Z} / \tilde{X}} \cong \rho^{*} \mathscr{N}_{Z / X} \otimes \mathcal{O}_{\tilde{Z}}\left(-E^{\prime}\right)$; if $Z$ and $Y$ intersect transversally in the sense that $\mathscr{N}_{Y, Z / X}=0$, then $\mathscr{N}_{\tilde{Z} / \tilde{X}} \cong \rho^{*} \mathscr{N}_{Z / X}$.

Proof. Applying Lemma 4.5 twice gives the following commutative diagram

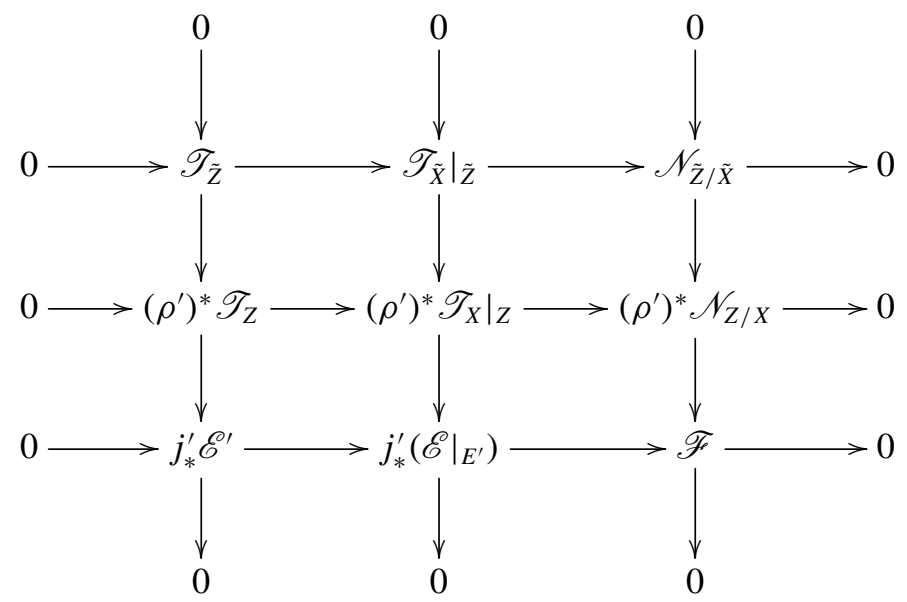

where $\mathscr{E}^{\prime \prime}:=\left(\pi^{\prime}\right)^{*} \mathscr{N}_{Y^{\prime} / Z} / \mathcal{O}_{E^{\prime}}(-1)$. Note that

$$
\left.\mathscr{E}\right|_{E^{\prime}} \cong \frac{\pi^{\prime *}\left(\left.\mathscr{N}_{Y / X}\right|_{Y^{\prime}}\right)}{\mathcal{O}_{E^{\prime}}(-1)}
$$

Then one easily deduce from the last row of the above commutative diagram that

$$
\mathscr{F} \cong j_{*}^{\prime} \pi^{\prime *} \mathscr{N}_{Y, Z / X}
$$

and hence the lemma follows.

Thirdly, the following lemma explains how the geometric projectivization of the inclusion of a subbundle into a bundle behaves with respect to the induced gradings on the Chow rings.

LEMMA 4.7. Let $Y$ be a smooth projective variety endowed with a self-dual multiplicative Chow-Künneth decomposition. Let $\mathscr{F}$ be a vector bundle on $Y$ such that $\operatorname{ch}(\mathscr{F}) \in \mathrm{CH}^{*}(Y)_{0}$. Let $\mathscr{F}^{\prime}$ be a subbundle of $\mathscr{F}$ such that $\operatorname{ch}\left(\mathscr{F}^{\prime}\right) \in$ $\mathrm{CH}^{*}(Y)_{0}$. Let $r+1=\mathrm{rk} \mathscr{F}$ and $r^{\prime}+1=\mathrm{rk} \mathscr{F}^{\prime}$. Then the natural embedding morphism $\varphi: \mathbb{P}\left(\mathscr{F}^{\prime}\right) \hookrightarrow \mathbb{P}(\mathscr{F})$ is of pure grade 0 , where both $\mathbb{P}(\mathscr{F})$ and $\mathbb{P}\left(\mathscr{F}^{\prime}\right)$ are given the self-dual multiplicative Chow-Künneth decomposition of a projective bundle as in Proposition 3.3. 
Proof. We first prove the weaker conclusion that

$$
\begin{aligned}
& \varphi^{*} \mathrm{CH}^{p}\left(\mathbb{P}\left(\mathscr{F}^{\prime}\right)\right)_{s} \subseteq \mathrm{CH}^{p}(\mathbb{P}(\mathscr{F}))_{s}, \\
& \varphi_{*} \mathrm{CH}^{p}\left(\mathbb{P}\left(\mathscr{F}^{\prime}\right)\right)_{s} \subseteq \mathrm{CH}^{p+\delta}(\mathbb{P}(\mathscr{F}))_{s},
\end{aligned}
$$

where $\delta=r-r^{\prime}$. Let $\xi$ (respectively $\xi^{\prime}$ ) be the first Chern class of the relative $\mathcal{O}(1)$-bundle on $\mathbb{P}(\mathscr{F})$ (respectively $\mathbb{P}\left(\mathscr{F}^{\prime}\right)$ ). Then we have $\xi^{\prime}=\varphi^{*} \xi$. Let $\pi$ : $\mathbb{P}(\mathscr{F}) \rightarrow Y$ and $\pi^{\prime}: \mathbb{P}\left(\mathscr{F}^{\prime}\right) \rightarrow Y^{\prime}$ be the two morphisms. With these notations, we have

$$
\varphi^{*}\left(\xi^{l} \cdot \pi^{*} \mathrm{CH}^{p-l}(Y)_{s}\right)=\xi^{\prime l} \cdot \pi^{\prime *} \mathrm{CH}^{p-l}(Y)_{s} .
$$

Together with the explicit description (16) of the graded components of the Chow ring of a projective bundle, this implies that $\varphi^{*}$ is compatible with the gradings on the Chow rings. For $\alpha \in \mathrm{CH}^{p-l}(Y)_{s}$, a direct computation yields

$$
\varphi_{*}\left(\xi^{\prime l} \cdot \pi^{\prime *} \alpha\right)=\varphi_{*} \varphi^{*}\left(\xi^{l} \cdot \pi^{*} \alpha\right)=\left[\mathbb{P}\left(\mathscr{F}^{\prime}\right)\right] \cdot \xi^{l} \cdot \pi^{*} \alpha .
$$

Thus to show the compatibility of $\varphi_{*}$ with the gradings, one only needs to verify that $\left[\mathbb{P}\left(\mathscr{F}^{\prime}\right)\right]$ belongs to $\mathrm{CH}^{r}(\mathbb{P}(\mathscr{F}))_{0}$ as a cycle on $\mathbb{P}(\mathscr{F})$. But then this is clear since $\mathrm{P}\left(\mathscr{F}^{\prime}\right)$ can be defined as the vanishing locus of a global section of the bundle $\pi^{*}\left(\mathscr{F} / \mathscr{F}^{\prime}\right) \otimes \mathcal{O}(1)$ on $\mathbb{P}(\mathscr{F})$. Hence the cycle class of $\mathbb{P}\left(\mathscr{F}^{\prime}\right)$ is equal to the top Chern class of $\pi^{*}\left(\mathscr{F} / \mathscr{F}^{\prime}\right) \otimes \mathcal{O}(1)$, which sits in $\mathrm{CH}^{r}(\mathbb{P}(\mathscr{F}))_{0}$ since all the Chern classes of $\mathscr{F} / \mathscr{F}^{\prime}$ are in the graded-0 part. Indeed, denoting $c_{i}=c_{i}\left(\mathscr{F} / \mathscr{F}^{\prime}\right)$, the cycle class of $\mathbb{P}\left(\mathscr{F}^{\prime}\right)$ can be expressed as

$$
\left[\mathbb{P}\left(\mathscr{F}^{\prime}\right)\right]=\xi^{\delta}+\pi^{*} c_{1} \cdot \xi^{\delta-1}+\cdots+\pi^{*} c_{\delta-1} \cdot \xi+\pi^{*} c_{\delta}, \quad \text { in } \mathrm{CH}^{*}(\mathbb{P}(\mathscr{F}))_{0} .
$$

The product $\mathrm{P}\left(\mathscr{F}^{\prime}\right) \times \mathbb{P}(\mathscr{F})$ can be viewed as the projectivization of $p_{2}^{*} \mathscr{F}$ on $\mathbb{P}\left(\mathscr{F}^{\prime}\right) \times X$ and $\mathbb{P}\left(\mathscr{F}^{\prime}\right) \times \mathbb{P}\left(\mathscr{F}^{\prime}\right)$ is the projectivization of the subbundle $p_{2}^{*} \mathscr{F}^{\prime} \subseteq$ $p_{2}^{*} \mathscr{F}$. The same argument shows that the action of the morphism

$$
\operatorname{id} \times \varphi: \mathbb{P}\left(\mathscr{F}^{\prime}\right) \times \mathbb{P}\left(\mathscr{F}^{\prime}\right) \longrightarrow \mathbb{P}\left(\mathscr{F}^{\prime}\right) \times \mathbb{P}(\mathscr{F})
$$

on the Chow groups is compatible with the gradings. In particular

$$
\Gamma_{\varphi}=(\mathrm{id} \times \varphi)_{*} \Delta_{\mathbb{P}\left(\mathscr{F}^{\prime}\right)}
$$

is of pure grade 0 .

We are now in a position to prove Proposition 4.4.

Proof of Proposition 4.4. That $\mathrm{Bl}_{Y}(\mathcal{S})$ is complete is obvious. Indeed, take $Y_{1}$, $Y_{2} \in \mathcal{S}$ which are not contained in $Y$ and let $\tilde{Y}_{1}, \tilde{Y}_{2} \in \mathrm{Bl}_{Y}(\mathcal{S})$ be their strict transforms. If $Y^{\prime}=Y_{1} \cap Y_{2}$ is not contained in $Y$, then the strict transform $\tilde{Y}^{\prime}$ of 
$Y^{\prime}$ is the intersection of $\tilde{Y}_{1}$ and $\tilde{Y}_{2}$ and $\tilde{Y}^{\prime} \in \mathrm{Bl}_{Y}(\mathcal{S})$; if $Y^{\prime} \subset Y$, then $\tilde{Y}_{1}$ and $\tilde{Y}_{2}$ do not meet each other since otherwise $\mathscr{T}_{Y_{1}}$ and $\mathscr{T}_{Y_{2}}$ do not intersect in constant rank and hence $Y^{\prime}$, as a scheme-theoretic intersection, is not reduced. Note that here we use the condition (ii) of completeness of $\mathcal{S}$ in an essential way; see Definition 4.1.

Let $Y_{j} \in \mathcal{S}$ be such that $Y_{j}$ is not contained in $Y$. By completeness of $\mathcal{S}$, we see that $Y_{j} \cap Y \in \mathcal{S}$. The strict transform $\tilde{Y}_{j}$ of $Y_{j}$ is the blow-up of $Y_{j}$ along $Y_{j} \cap Y$. Since $\mathcal{S}$ is admissible, we conclude from Proposition 4.3 that $\tilde{Y}_{j}$ is smooth and naturally endowed with a self-dual multiplicative Chow-Künneth decomposition.

We first prove that $\mathrm{Bl}_{Y}(\mathcal{S})$ is admissible for the special case

$$
\mathcal{S}=\left\{X, Y, Z, Y^{\prime}=Y \cap Z\right\},
$$

where $Z$ is not contained in $Y$. In this case, we still use $\tilde{X}$ to denote the blow-up of $X$ with center $Y$. Let $\tilde{Z} \subset \tilde{X}$ be the strict transform of $Z$. Then $\tilde{Z}$ is simply the blow-up $\mathrm{Bl}_{Y^{\prime}}(Z)$ of $Z$ along $Y^{\prime}$ and we have

$$
\mathrm{Bl}_{Y}(\mathcal{S})=\{\tilde{Z}, \tilde{X}\} .
$$

We have seen that both $\tilde{Z}$ and $\tilde{X}$ have a self-dual multiplicative Chow-Künneth decomposition. To prove the proposition, we still need to verify the following conditions: (a) the Chern classes of $\mathscr{N}_{\tilde{Z} / \tilde{X}}$ sit in $\mathrm{CH}^{*}(\tilde{Z})_{0}$; (b) the inclusion morphism $\tilde{Z} \hookrightarrow \tilde{X}$ is of pure grade 0 . To do that, we will first prove (a) and a weaker conclusion $\left(\mathrm{b}^{\prime}\right)$ the push-forward via the embedding $j_{\tilde{Z}}: \tilde{Z} \hookrightarrow \tilde{X}$ are compatible with the gradings of the Chow rings. Consider the following diagram

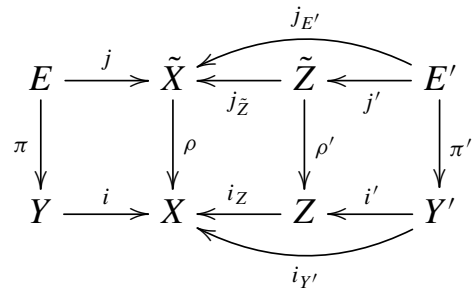

where the two extremal squares are the blow-up squares.

It follows from Lemma 4.6 that

$$
\operatorname{ch}\left(\mathscr{N}_{\tilde{Z} / \tilde{X}}\right)=\left(\rho^{\prime}\right)^{*} \operatorname{ch}\left(\mathscr{N}_{Z / X}\right)-\operatorname{ch}\left(j_{*}^{\prime} \pi^{* *} \mathscr{N}_{Y, Z / X}\right) .
$$

Applying the Grothendieck-Riemann-Roch theorem to the morphism $j^{\prime}$ yields

$$
\operatorname{ch}\left(j_{*}^{\prime} \pi^{\prime *} \mathscr{N}_{Y, Z / X}\right)=j_{*}^{\prime}\left(\frac{\pi^{\prime *} \operatorname{ch}\left(\mathscr{N}_{Y, Z / X}\right)}{\operatorname{td}\left(\mathscr{N}_{E^{\prime} / \tilde{Z}}\right)}\right) .
$$


We also note that there is a short exact sequence

$$
0 \longrightarrow \mathscr{N}_{Y^{\prime} / Y} \oplus \mathscr{N}_{Y^{\prime} / Z} \longrightarrow \mathscr{N}_{Y^{\prime} / X} \longrightarrow \mathscr{N}_{Y, Z / X} \longrightarrow 0 .
$$

By assumption the set $\mathcal{S}$ is admissible so that the Chern classes of $\mathscr{N}_{Y^{\prime} / Z}, \mathscr{N}_{Y^{\prime} / Y}$ and $\mathscr{N}_{Y^{\prime} / X}$ are in $\mathrm{CH}^{*}\left(Y^{\prime}\right)_{0}$. Then we conclude that

$$
\operatorname{ch}\left(\mathscr{N}_{Y, Z / X}\right) \in \mathrm{CH}^{*}\left(Y^{\prime}\right)_{0} .
$$

Using the explicit description (17) of the graded pieces of the Chow ring of a smooth blow-up and the compatibility of $j_{*}^{\prime}$ with the grading of the Chow groups, we see that $\operatorname{ch}\left(j_{*}^{\prime} \pi^{\prime *} \mathscr{N}_{Y, Z / X}\right)$ sits in $\mathrm{CH}^{*}(\tilde{Z})_{0}$. Hence it follows that $\operatorname{ch}\left(\mathscr{N}_{\tilde{Z} / \tilde{X}}\right)$ sits in $\mathrm{CH}^{*}(\tilde{Z})_{0}$. This establishes (a).

The decomposition of the Chow groups of $\tilde{Z}$ is given, as in Proposition 3.4, by

$$
\mathrm{CH}^{p}(\tilde{Z})_{s}=\left(\rho^{\prime}\right)^{*} \mathrm{CH}^{p}(Z)_{s} \oplus\left(\bigoplus_{l=0}^{\operatorname{codim}_{Z} Y^{\prime}-2} j_{*}^{\prime}\left(\xi^{\prime l} \cdot \pi^{\prime *} \mathrm{CH}^{p-l-1}\left(Y^{\prime}\right)_{s}\right)\right) .
$$

Similarly the decomposition of the Chow groups of $\tilde{X}$ is given by

$$
\mathrm{CH}^{p}(\tilde{X})_{s}=\rho^{*} \mathrm{CH}^{p}(X)_{s} \oplus\left(\bigoplus_{l=0}^{\operatorname{codim}_{X} Y-2} j_{*}\left(\xi^{l} \cdot \pi^{*} \mathrm{CH}^{p-l-1}(Y)_{s}\right)\right) .
$$

Let $f: Y^{\prime} \hookrightarrow Y$ be the inclusion morphism. For any $\alpha \in \mathrm{CH}_{k}(Z)$, we claim that $\rho^{*}\left(j_{Z}\right)_{*} \alpha=\left(j_{\tilde{Z}}\right)_{*} \rho^{\prime *} \alpha+j_{*}\left(c(\mathscr{E}) \cap \pi^{*} f_{*}\left(s\left(Y^{\prime}, Z\right) \cdot i^{\prime *} \alpha\right)\right)_{k}, \quad$ with $\mathscr{E}=\frac{\pi^{*} \mathscr{N}_{Y / X}}{\mathcal{O}_{E}(-1)}$,

where $(-)_{k}$ means taking the $k$-dimensional component and $s\left(Y^{\prime}, Z\right)$ is the Segre class of $Y^{\prime}$ in $Z$. (We refer to [6, Section 4.2] for the definition and properties of Segre classes.) This claim can be proved as follows. We may assume that $\alpha$ is represented by a closed subvariety $W \subseteq Z$ which intersects $Y^{\prime}$ properly. Let $\tilde{W} \subseteq \tilde{Z}$ be the strict transform of $W$ and set $W^{\prime}:=W \cap Y^{\prime}$. Since $W$ intersects $Y^{\prime}$ properly, we see that

$$
\rho^{\prime *} \alpha=\tilde{W}, \quad \text { in } \mathrm{CH}_{k}(\tilde{Z}) .
$$

We apply [6, Theorem 6.7] to $W$ with respect to the blow-up morphism $\rho$ and get

$$
\rho^{*}\left(j_{Z}\right)_{*} \alpha=\left(j_{\tilde{Z}}\right)_{*} \tilde{W}+j_{*}\left(c(\mathscr{E}) \cap \pi^{*} f_{*}\left(j_{W^{\prime}}\right)_{*} s\left(W^{\prime}, W\right)\right)_{k},
$$

where $j_{W^{\prime}}: W^{\prime} \rightarrow Y^{\prime}$. Since $W$ intersects $Y^{\prime}$ properly, we have an equality $s\left(W^{\prime}, W\right)=\left(j_{W^{\prime}}\right)^{*} s\left(Y^{\prime}, Z\right)$. Thus we obtain

$$
\begin{aligned}
\rho^{*}\left(j_{Z}\right)_{*} \alpha & =\left(j_{\tilde{Z}}\right)_{*} \tilde{W}+j_{*}\left(c(\mathscr{E}) \cap \pi^{*} f_{*}\left(j_{W^{\prime}}\right)_{*}\left(j_{W^{\prime}}\right)^{*} s\left(Y^{\prime}, Z\right)\right)_{k} \\
& =\left(j_{\tilde{Z}}\right)_{*} \tilde{W}+j_{*}\left(c(\mathscr{E}) \cap \pi^{*} f_{*}\left(\left[W^{\prime}\right] \cdot s\left(Y^{\prime}, Z\right)\right)\right)_{k} \\
& =\left(j_{\tilde{Z}}\right)_{*} \rho^{\prime *} \alpha+j_{*}\left(c(\mathscr{E}) \cap \pi^{*} f_{*}\left(i^{*} \alpha \cdot s\left(Y^{\prime}, Z\right)\right)\right)_{k},
\end{aligned}
$$


which settles the claim. We also note that $s\left(Y^{\prime}, Z\right)$ is the inverse of $c\left(\mathscr{N}_{Y^{\prime} / Z}\right)$ and hence lies in the graded-0 part. It is also clear that $c(\mathscr{E})$ lies in the graded-0 part. If $\alpha \in \mathrm{CH}^{p}(Z)_{s}$, then equation (23) implies that

$$
\left(j_{\tilde{Z}}\right)_{*} \rho^{\prime *} \alpha \in \mathrm{CH}^{p+\operatorname{dim} X-\operatorname{dim} Z}(\tilde{X})_{s} .
$$

If $\alpha \in \mathrm{CH}^{p-l-1}\left(Y^{\prime}\right)_{s}$, then

$$
\begin{aligned}
\left(j_{\tilde{Z}}\right)_{*} j_{*}^{\prime}\left(\xi^{\prime l} \cdot \pi^{\prime *} \alpha\right) & =\left(j_{\tilde{Z}}\right)_{*} j_{*}^{\prime}\left(j^{\prime *}\left(-E^{\prime}\right)^{l} \cdot \pi^{\prime *} \alpha\right) \\
& =\left(j_{\tilde{Z}}\right)_{*}\left(\left(-E^{\prime}\right)^{l} \cdot j_{*}^{\prime} \pi^{\prime *} \alpha\right) \\
& =\left(j_{\tilde{Z}}\right)_{*}\left(\left(j_{\tilde{Z}}\right)^{*}(-E)^{l} \cdot j_{*}^{\prime} \pi^{\prime *} \alpha\right) \\
& =(-E)^{l} \cdot\left(j_{\tilde{Z}}\right)_{*} j_{*}^{\prime} \pi^{\prime *} \alpha .
\end{aligned}
$$

Let $j_{E^{\prime} / E}: E^{\prime} \hookrightarrow E$ be the embedding morphism. According to Lemma 4.7 the push-forward map $\left(j_{E^{\prime} / E}\right)_{*}$ respects the gradings of the Chow groups. Thus we obtain

$$
\left(j_{\tilde{Z}}\right)_{*} j_{*}^{\prime}\left(\xi^{\prime l} \cdot \pi^{\prime *} \alpha\right)=(-E)^{l} \cdot\left(j_{\tilde{Z}}\right)_{*} j_{*}^{\prime} \pi^{\prime *} \alpha=(-E)^{l} \cdot j_{*}\left(j_{E^{\prime} / E}\right)_{*} \pi^{\prime *} \alpha \in \mathrm{CH}^{*}(\tilde{X})_{s} .
$$

This proves $\left(b^{\prime}\right)$.

We now deal with the stronger condition (b). Note that the set

$$
\mathcal{S}^{\prime}=\left\{\tilde{Z} \times X, \tilde{Z} \times Y, \tilde{Z} \times Z, \tilde{Z} \times Y^{\prime}\right\}
$$

is admissible and hence the same argument above shows that the push-forward by the inclusion $1 \times j_{\tilde{Z}}: \tilde{Z} \times \tilde{Z} \hookrightarrow \tilde{Z} \times \tilde{X}$ respects the grading on Chow groups. Hence the graph of $j_{\tilde{Z}}$, which equals the push-forward of $\Delta_{\tilde{Z}}$, is of pure grade 0 . This establishes (b).

We now move on to the general case. Let $Z_{1} \subset Z_{2}$ where $Z_{1}, Z_{2} \in \mathcal{S}$ are such that none of them is contained in $Y$. Then the conditions for the admissibility for the inclusion $\tilde{Z}_{1} \subset \tilde{Z}_{2}$ can be checked by applying the above special case to

$$
\mathcal{S}^{\prime \prime}=\left\{W^{\prime}=Y \cap Z_{1}, W=Y \cap Z_{2}, Z_{1}, Z_{2}\right\} .
$$

More precisely, we replace $Y$ by $W, Y^{\prime}$ by $W^{\prime}, Z$ by $Z_{1}$ and $X$ by $Z_{2}$. This settles the proposition.

\section{Resolving the rational map $X^{3}-\rightarrow X^{[3]}$}

Let $X$ be a smooth projective variety of dimension $d$. Our strategy for proving Theorem 1 consists in desingularizing the rational map $X^{3} \rightarrow-X^{[3]}$ by blowing up $X^{3}$ several times in such a way that the variety obtained after each blow-up has a multiplicative Chow-Künneth decomposition, and in such a way that after the final blow-up necessary to resolve the map the Chow-Künneth decomposition descends to a Chow-Künneth decomposition of $X^{[3]}$. 
5.1. A stratification of $X^{[3]}$. In this subsection, we explicitly describe some canonical subvarieties of $X^{[3]}$. These subvarieties will play important roles in the study of the resulting morphism after resolving the rational map $X^{3} \rightarrow X^{[3]}$. Such subvarieties also appear in the cellular decomposition considered in [4] and earlier works.

A general point of $X^{[3]}$ corresponds to a reduced length-3 subscheme of $X$, or equivalently to an unordered set of three distinct points of $X$. When two of those points coincide, one gets a nonreduced subscheme of length 3 . For any point $\xi \in X^{[3]}$, we denote $Z_{\xi} \subset X$ the corresponding closed subscheme of $X$. Let

$$
B_{1}:=\left\{\xi \in X^{[3]}: Z_{\xi} \text { is a nonreduced subscheme of } X\right\} .
$$

Then $B_{1}$ is a divisor on $X^{[3]}$. Let

$$
B_{2}:=\left\{\xi \in X^{[3]}: Z_{\xi} \text { is supported on a single point of } X\right\} .
$$

Assume that $Z=Z_{\xi} \subset X$ is a length-3 subscheme supported at a single point $x \in X$. Let $\mathscr{I} \subset \mathcal{O}_{X}$ be the ideal sheaf of $Z$ and let $m_{x} \subset \mathcal{O}_{X}$ be the maximal ideal corresponding to the point $x$. There are two cases.

The first case is $m_{x}^{2} \subset \mathscr{I}$, or equivalently the image of $\mathscr{I}$ in $m_{x} / m_{x}^{2}$ has dimension $d-2$. In this case, $\mathscr{I}$ determines a $(d-2)$-dimensional subspace of $\Omega_{X, x}$ which, by dualization, gives a two-dimensional subspace $\mathscr{V}_{x}$ of $\mathscr{T}_{X, x}$. The point $\xi$ is actually determined by $\mathscr{V}_{x}$ as follows. Take some local coordinates $\left\{t_{1}, t_{2}, t_{3}, \ldots, t_{d}\right\}$ of $X$ at the point $x$ such that $\mathscr{V}_{x}$ is the kernel of $\left\{\mathrm{d} t_{3}, \ldots, \mathrm{d} t_{d}\right\}$ at the point $x$. Then we have

$$
\mathscr{I}=\left(t_{1}^{2}, t_{1} t_{2}, t_{2}^{2}, t_{3}, \ldots, t_{d}\right)
$$

on an open neighborhood of $x$. If we define

$$
B_{3}=\left\{\xi \in X^{[3]}: Z_{\xi} \text { is supported in a single point } x \text { such that } m_{x}^{2} \subseteq \mathscr{I}_{Z_{\xi}}\right\},
$$

then we have the natural isomorphism

$$
B_{3} \cong \operatorname{Gr}\left(2, \mathscr{T}_{X}\right)
$$

The second case is when the image of $\mathscr{I}$ in $m_{x} / m_{x}^{2}$ has dimension $d-1$. Dually, this determines a one-dimensional subspace of $\mathscr{T}_{X, x}$. Pick local coordinates $\left\{t_{1}, \ldots, t_{d}\right\}$ of $X$ at $x$ such that $\mathrm{d} t_{1}, \ldots, \mathrm{d} t_{d-1}$ generate the image of $\mathscr{I}$ in $m_{x} / m_{x}^{2}$. Then we have

$$
\mathscr{I}=\left(t_{1}+a_{1} t_{d}^{2}, t_{2}+a_{2} t_{d}^{2}, \ldots, t_{d-1}+a_{d-1} t_{d}^{2}, t_{d}^{3}\right)
$$


for some $a_{1}, a_{2}, \ldots, a_{d-1}$ in the base field. This implies that $B_{2} \backslash B_{3}$ is an $\mathbb{A}^{d-1}$ bundle over $\mathbb{P}\left(\mathscr{T}_{X}\right)$. Globalizing the above picture yields

$$
B_{2} \backslash B_{3} \cong \mathbb{P}(\mathscr{E}) \backslash \mathbb{P}\left(\mathscr{T}_{\mathbb{P}\left(\mathscr{T}_{X}\right) / X}\right)
$$

where $\mathscr{E}$ is a locally free sheaf of rank $d$ on $\mathbb{P}\left(\mathscr{T}_{X}\right)$ and $\mathscr{T}_{\mathbb{P}\left(\mathscr{T}_{X}\right) / X} \subset \mathscr{E}$ is naturally a subbundle; see (39).

5.2. Desingularizing the rational map $X^{\mathbf{3}} \rightarrow X^{[3]}$. In this subsection, we first describe how to resolve the map $X^{3}-\rightarrow X^{[3]}$ for any smooth projective variety $X$. Then we move on to a careful study of the resulting morphism $p: X_{3} \rightarrow X^{[3]}$ and this will be essential to descend the Chow-Künneth decomposition from $X_{3}$ to $X^{[3]}$.

Consider the following smooth closed subvarieties of $X^{3}$ :

$$
\begin{aligned}
\Delta_{12} & :=\{(x, x, y): x, y \in X\} \subset X^{3}, \\
\Delta_{23} & :=\{(y, x, x): x, y \in X\} \subset X^{3}, \\
\Delta_{13} & :=\{(x, y, x): x, y \in X\} \subset X^{3}, \\
\Delta_{123} & :=\{(x, x, x): x \in X\} \subset X^{3} .
\end{aligned}
$$

In practice, we also think of $\Delta_{i j}$ as a morphism

$$
\phi_{i j}: X \times X \rightarrow X^{3},
$$

such that $\phi_{i j}(x, y)$ is the point whose $i$ th and $j$ th coordinates are $x$ and the remaining coordinate is $y$. We denote $X_{1}$ the blow-up of $X_{0}:=X^{3}$ along the small diagonal $\Delta_{123}$ and $\tilde{\Delta}_{i j}$ the strict transforms of the big diagonals $\Delta_{i j}$. Note that $\tilde{\Delta}_{12}, \tilde{\Delta}_{23}$ and $\tilde{\Delta}_{13}$ are pairwise disjoint as subvarieties of $X_{1}$. We then denote $X_{2}$ the blow-up of $X_{1}$ along the disjoint union of the $\tilde{\Delta}_{i j}$. If $X$ is a surface, then the rational map $X_{2} \rightarrow X^{[3]}$ is already a morphism. If $\operatorname{dim} X \geqslant 3$ then, as explained in the proof of [16, Lemma 3.12], the rational map $X_{2}-\rightarrow X^{[3]}$ is not yet a morphism, but it does become a morphism after one more blow-up that we describe now.

Let $E_{1} \subset X_{1}$ be the exceptional divisor of the blow-up $\rho_{1}: X_{1} \rightarrow X_{0}$, where $X_{0}:=X^{3}$, along the small diagonal $Y_{0}:=\Delta_{123}$. The tangent bundle $\mathscr{T}_{X_{0}}$ restricted to $Y_{0}=\Delta_{123} \cong X$ is naturally isomorphic to $\mathscr{T}_{X} \oplus \mathscr{T}_{X} \oplus \mathscr{T}_{X}$. The tangent bundle 
$\mathscr{T}_{Y_{0}} \cong \mathscr{T}_{X}$ maps diagonally into $\left.\mathscr{T}_{X_{0}}\right|_{Y_{0}}$. Let $\mathscr{N}_{Y_{0} / X_{0}}$ be the normal bundle of $Y_{0}$ in $X_{0}$. There is a commutative diagram

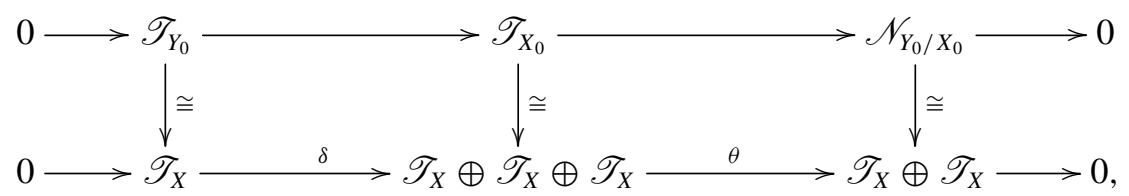

where $\delta(a)=(a, a, a)$ and $\theta(a, b, c)=(a-b, b-c)$. Hence we get an identification

$$
E_{1} \cong \mathbb{P}\left(\mathscr{T}_{X} \oplus \mathscr{T}_{X}\right) .
$$

Let $W^{\prime} \subset \mathbb{P}\left(\mathscr{T}_{X} \oplus \mathscr{T}_{X}\right)$ be the subvariety of all vectors $(a, b)$ such that $a$ and $b$ are colinear in $\mathscr{T}_{X}$. First we note that there is a natural isomorphism

$$
\mathbb{P}^{1} \times \mathbb{P}\left(\mathscr{T}_{X}\right) \rightarrow W^{\prime}, \quad([s: t],[v]) \mapsto[(s v, t v)],
$$

where $[s: t]$ are the homogeneous coordinates of $\mathbb{P}^{1}$ and $[v] \in \mathbb{P}\left(\mathscr{T}_{X}\right)$ is the class of a nonzero vector $v \in \mathscr{T}_{X}$. Under the isomorphism $\mathscr{N}_{Y_{0} / X_{0}} \cong \mathscr{T}_{X} \oplus \mathscr{T}_{X}$, the variety $W^{\prime}$ corresponds to a smooth closed subvariety

$$
W \subset E_{1}=\mathbb{P}\left(\mathscr{N}_{Y_{0} / X_{0}}\right) \subset X_{1},
$$

and there is an isomorphism

$$
W \cong \mathbb{P}^{1} \times \mathbb{P}\left(\mathscr{T}_{X}\right)
$$

Let $\tilde{W}$ be the strict transform of $W$ under the blow-up $X_{2} \rightarrow X_{1}$. Writing $X_{3}$ for the smooth blow-up of $X_{2}$ along $\tilde{W}$, we then have (see also Proposition 5.2)

Proposition 5.1. The rational map $p: X_{3} \rightarrow X^{[3]}$ is a generically finite morphism. Moreover, the natural action of the symmetric group $\mathfrak{S}_{3}$ on $X^{3}$ lifts to $X_{3}$ and the morphism $p: X_{3} \rightarrow X^{[3]}$ factors through the quotient.

Proof. We refer to the proof of [16, Lemma 3.12]. This can also be seen from the description of the blow-ups below.

For our purpose, we need to understand the morphism $X_{3} \rightarrow X^{[3]}$ in more depth.

The natural subvarieties of $X_{1}$ have explicit geometric descriptions. For example, we first have

$$
\tilde{\Delta}_{i j} \cong \mathrm{Bl}_{\Delta_{X}}(X \times X) \text {. }
$$


Note that $\tilde{\Delta}_{i j} \cap E_{1} \cong \mathbb{P}\left(\mathscr{T}_{X}\right)$ for all $i, j$ and under the identification (30) these can be described as follows:

$$
\begin{array}{lll}
W_{12}:=\tilde{\Delta}_{12} \cap E_{1} \subset E_{1}: & \mathbb{P}\left(\mathscr{T}_{X}\right) \hookrightarrow \mathbb{P}\left(\mathscr{T}_{X} \oplus \mathscr{T}_{X}\right), & {[v] \mapsto[0, v] ;} \\
W_{23}:=\tilde{\Delta}_{23} \cap E_{1} \subset E_{1}: & \mathbb{P}\left(\mathscr{T}_{X}\right) \hookrightarrow \mathbb{P}\left(\mathscr{T}_{X} \oplus \mathscr{T}_{X}\right), & {[v] \mapsto[v, 0] ;} \\
W_{13}:=\tilde{\Delta}_{13} \cap E_{1} \subset E_{1}: & \mathbb{P}\left(\mathscr{T}_{X}\right) \hookrightarrow \mathbb{P}\left(\mathscr{T}_{X} \oplus \mathscr{T}_{X}\right), & {[v] \mapsto[v,-v] .}
\end{array}
$$

From the definition of $W$, one immediately sees that $W_{i j} \subset W$. Under the identification (32) we have

$W_{12}=[0: 1] \times \mathbb{P}\left(\mathscr{T}_{X}\right), \quad W_{23}=[1: 0] \times \mathbb{P}\left(\mathscr{T}_{X}\right), \quad W_{13}=[1:-1] \times \mathbb{P}\left(\mathscr{T}_{X}\right)$.

Let $E_{i j} \subset X_{2}$ be the exceptional divisor sitting above $\tilde{\Delta}_{i j}$. Thinking of $\Delta_{i j}$ as being isomorphic to $X \times X$ as in (28), we have

$$
\mathscr{N}_{\Delta_{i j} / X_{0}}=p_{1}^{*} \mathscr{T}_{X},
$$

where $p_{1}: X \times X \rightarrow X$ is the projection onto the first factor. It follows from Lemma 4.6 that

$$
\mathscr{N}_{\tilde{\Delta}_{i j} / X_{1}} \cong \tilde{p}_{1}^{*} \mathscr{T}_{X} \otimes \mathcal{O}_{\tilde{\Delta}_{i j}}\left(-E_{\Delta}\right)
$$

where we identify $\tilde{\Delta}_{i j}$ with $\mathrm{Bl}_{\Delta_{X}}(X \times X)$ and $\tilde{p}_{1}$ is the composition of $p_{1}: X \times$ $X \rightarrow X$ with the blow-up morphism $\mathrm{Bl}_{\Delta_{X}}(X \times X) \rightarrow X \times X$. It follows that $E_{i j} \cong \mathbb{P}\left(\tilde{p}_{1}^{*} \mathscr{T}_{X}\right)$, or equivalently $E_{i j}$ is the blow-up of $\mathbb{P}\left(\mathscr{T}_{X}\right) \times X$ along the graph of $\pi: \mathbb{P}\left(\mathscr{T}_{X}\right) \rightarrow X$. Let $E_{1}^{\prime} \subset X_{2}$ be the strict transform of $E_{1}$. Then $E_{1}^{\prime}$ is the blow-up of $E_{1}$ along the disjoint union of the subvarieties $W_{i j}$. One easily sees that

$$
E_{1, i j}^{\prime}:=E_{1}^{\prime} \cap E_{i j} \cong \mathbb{P}\left(\mathscr{T}_{X}\right) \times_{X} \mathbb{P}\left(\mathscr{T}_{X}\right) .
$$

Furthermore, $E_{1, i j}^{\prime}$ is the exceptional divisor of the blow-up $E_{1}^{\prime} \rightarrow E_{1}$ and also the exceptional divisor of the blow-up $E_{i j} \cong \mathrm{Bl}_{\Gamma_{\pi}}\left(\mathbb{P}\left(\mathscr{T}_{X}\right) \times X\right) \rightarrow \mathbb{P}\left(\mathscr{T}_{X}\right) \times X$. Each blow-up contracts one of the two $\mathbb{P}\left(\mathscr{T}_{X}\right)$-factors respectively. We also see that the strict transform $\tilde{W} \subset E_{1}^{\prime}$ is isomorphic to $W$.

Under the identifications (30) and (32), the inclusion $W \subset E_{1}$ is induced by

$$
p_{1}^{*} \mathcal{O}_{\mathbb{P}^{1}}(-1) \otimes p_{2}^{*} \mathcal{O}_{\mathbb{P}\left(\mathscr{T}_{X}\right)}(-1) \hookrightarrow \mathbb{C}^{2} \otimes \mathscr{T}_{X} \cong \mathscr{T}_{X}^{\oplus 2},
$$

where $p_{i}$ are the two projections from $W=\mathbb{P}^{1} \times \mathbb{P}\left(\mathscr{T}_{X}\right)$ to its factors. In particular,

$$
\left.\mathcal{O}_{E_{1}}(1)\right|_{W}=p_{1}^{*} \mathcal{O}_{\mathbb{P}^{1}}(1) \otimes p_{2}^{*} \mathcal{O}_{\mathbb{P}\left(\mathscr{T}_{X}\right)}(1) .
$$

We have the following natural isomorphisms

$$
\mathscr{T}_{W / X} \cong \mathscr{T}_{\mathbb{P}^{1}} \oplus \mathscr{T}_{\mathbb{P}\left(\mathscr{T}_{X}\right) / X}=\frac{\mathbb{C}^{2} \otimes \mathcal{O}_{\mathbb{P}^{1}}(1)}{\mathcal{O}_{\mathbb{P}^{1}}} \oplus \frac{\mathscr{T}_{X} \otimes \mathcal{O}_{\mathbb{P}\left(\mathscr{T}_{X}\right)}(1)}{\mathcal{O}_{\mathbb{P}\left(\mathscr{T}_{X}\right)}}
$$




$$
\left.\mathscr{T}_{E_{1} / X}\right|_{W} \cong \frac{\left.\mathscr{T}_{X}^{\oplus 2} \otimes \mathcal{O}_{E_{1}}(1)\right|_{W}}{\mathcal{O}_{W}}=\frac{\mathbb{C}^{2} \otimes \mathcal{O}_{\mathbb{P}^{1}}(1) \otimes \mathscr{T}_{X} \otimes \mathcal{O}_{\mathbb{P}\left(\mathscr{T}_{X}\right)}(1)}{\mathcal{O}_{W}}
$$

Here all the sheaves are viewed as sheaves on $W$ via pulling back the corresponding ones. From this, we see that the normal bundle of $W$ in $E_{1}$ is identified as

$$
\mathscr{N}_{W / E_{1}} \cong p_{1}^{*} \mathscr{T}_{\mathbb{P}^{1}} \otimes p_{2}^{*} \mathscr{T}_{\mathbb{P}\left(\mathscr{T}_{X}\right) / X}
$$

Since $E_{1}^{\prime}$ is the blow-up of $E_{1}$ along $W_{i j}$, where $W_{i j}$ are divisors on $W$, we conclude by Lemma 4.6 that

$$
\mathscr{N}_{\tilde{W} / E_{1}^{\prime}}=\mathscr{N}_{W / E_{1}} \otimes \mathcal{O}_{W}\left(-W_{12}-W_{23}-W_{13}\right) \cong p_{1}^{*} \mathcal{O}_{\mathbb{P}^{1}}(-1) \otimes p_{2}^{*} \mathscr{T}_{\mathbb{P}\left(\mathscr{T}_{X}\right) / X}
$$

Since $E_{1}^{\prime}$ meets $\tilde{\Delta}_{i j}$ transversally, again by Lemma 4.6 , we see that $\mathscr{N}_{E_{1}^{\prime} / X_{2}}$ is isomorphic to the pull-back of $\mathscr{N}_{E_{1} / X_{1}}$. Hence we get

$$
\left.\left.\mathscr{N}_{E_{1}^{\prime} / X_{2}}\right|_{\tilde{W}} \cong \mathscr{N}_{E_{1} / X_{1}}\right|_{W}=\left.\mathcal{O}_{E_{1}}(-1)\right|_{W} \cong p_{1}^{*} \mathcal{O}_{\mathbb{P}^{1}}(-1) \otimes p_{2}^{*} \mathcal{O}_{\mathbb{P}\left(\mathscr{T}_{X}\right)}(-1)
$$

The normal bundle of $\tilde{W}$ in $X_{2}$ fits into the following short exact sequence

$$
\left.0 \longrightarrow \mathscr{N}_{\tilde{W} / E_{1}^{\prime}} \longrightarrow \mathscr{N}_{\tilde{W} / X_{2}} \longrightarrow \mathscr{N}_{E_{1}^{\prime} / X_{2}}\right|_{\tilde{W}} \longrightarrow 0 \text {. }
$$

By the isomorphisms (36) and (37), the short exact sequence can be rewritten as

$$
0 \longrightarrow p_{1}^{*} \mathcal{O}_{\mathbb{P}^{1}}(-1) \otimes p_{2}^{*} \mathscr{T}_{\mathbb{P}\left(\mathscr{T}_{X}\right) / X} \longrightarrow \mathscr{N}_{\tilde{W} / X_{2}} \longrightarrow p_{1}^{*} \mathcal{O}_{\mathbb{P}^{1}}(-1) \otimes p_{2}^{*} \mathcal{O}_{\mathbb{P}\left(\mathscr{T}_{X}\right)}(-1) \longrightarrow 0 .
$$

This defines an element in

$$
\begin{aligned}
& \operatorname{Ext}_{\mathcal{O}_{W}}^{1}\left(p_{1}^{*} \mathcal{O}_{\mathbb{P}^{1}}(-1) \otimes p_{2}^{*} \mathcal{O}_{\mathbb{P}\left(\mathscr{T}_{X}\right)}(-1), p_{1}^{*} \mathcal{O}_{\mathbb{P}^{1}}(-1) \otimes p_{2}^{*} \mathscr{T}_{\mathbb{P}\left(\mathscr{T}_{X}\right) / X}\right) \\
& \quad=\operatorname{Ext}_{\mathcal{O}_{\mathbb{P}\left(\mathscr{T}_{X}\right)}}\left(\mathcal{O}_{\mathbb{P}\left(\mathscr{T}_{X}\right)}(-1), \mathscr{T}_{\mathbb{P}\left(\mathscr{T}_{X}\right) / X}\right)
\end{aligned}
$$

Hence there is a locally free sheave $\mathscr{E}$ on $\mathrm{P}\left(\mathscr{T}_{X}\right)$ such that

$$
\mathscr{N}_{\tilde{W} / X_{2}} \cong p_{1}^{*} \mathcal{O}_{\mathbb{P}^{1}}(-1) \otimes p_{2}^{*} \mathscr{E} .
$$

Furthermore, the short exact sequence (38) shows that $\mathscr{E}$ naturally fits into the following short exact sequence

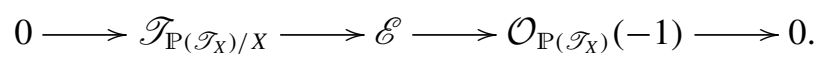

Let $E_{W} \subset X_{3}$ be the exceptional divisor of the blow-up $X_{3} \rightarrow X_{2}$. Then we have

$$
E_{W}=\mathbb{P}\left(\mathscr{N}_{\tilde{W} / X_{2}}\right)=\mathbb{P}^{1} \times \mathbb{P}(\mathscr{E}) .
$$


Let $E_{1}^{\prime \prime} \subset X_{3}$ be the strict transform of $E_{1}^{\prime} \subset X_{2}$. Note that $E_{1}^{\prime \prime}$ is the blow-up of $E_{1}^{\prime}$ along $\tilde{W}$ with exceptional divisor $E_{W}^{\prime \prime}=\mathbb{P}\left(\mathscr{N}_{\tilde{W} / E_{1}^{\prime}}\right)=\mathbb{P}^{1} \times \mathbb{P}\left(\mathscr{T}_{\mathbb{P}\left(\mathscr{T}_{X}\right) / X}\right)$. We also have $E_{W}^{\prime \prime}=E_{1}^{\prime \prime} \cap E_{W}$ and the inclusion $E_{W}^{\prime \prime} \subset E_{W}$ is induced by the subsheaf $\mathscr{T}_{\mathbb{P}\left(\mathscr{T}_{X}\right) / X} \subset \mathscr{E}$ in (39). The morphism $p: X_{3} \rightarrow X^{[3]}$ contracts the two divisors $E_{W}$ and $E_{1}^{\prime \prime}$ in the following way. The morphism $p: X_{3} \rightarrow X^{[3]}$ contracts $E_{1}^{\prime \prime}$ onto $B_{3} \cong \mathrm{G}\left(2, \mathscr{T}_{X}\right)$ and it contracts $E_{W} \backslash E_{W}^{\prime \prime}$ onto $B_{2} \backslash B_{3}$. Note that

$$
E_{W} \backslash E_{W}^{\prime \prime} \cong \mathbb{P}^{1} \times \mathbb{P}(\mathscr{E}) \backslash \mathbb{P}\left(\mathscr{T}_{\mathbb{P}\left(\mathscr{T}_{X}\right) / X}\right)
$$

and

$$
B_{2} \backslash B_{3} \cong \mathbb{P}(\mathscr{E}) \backslash \mathbb{P}\left(\mathscr{T}_{\mathbb{P}\left(\mathscr{T}_{X}\right) / X}\right) .
$$

The morphism $p$ simply contracts the $\mathbb{P}^{1}$-factor of $E_{W} \backslash E_{W}^{\prime \prime}$.

We summarize the above discussion in the following proposition.

Proposition 5.2. The morphism $p: X_{3} \rightarrow X^{[3]}$ contracts two divisors $E_{1}^{\prime \prime}$ and $E_{W}$ to $B_{3}$ and $B_{2}$, respectively. The ramification divisors outside $E_{1}^{\prime \prime} \cup E_{W}$ are $\tilde{E}_{i j}$ (strict transform of $E_{i j}$ ), $1 \leqslant i<j \leqslant 3$. Furthermore, setting $t_{12}=[0: 1]$, $t_{23}=[1: 0], t_{13}=[1:-1]$, we have

$$
\begin{aligned}
E_{W} \cap \tilde{E}_{i j} & =\left\{t_{i j}\right\} \times \mathbb{P}(\mathscr{E}) \hookrightarrow E_{W}=\mathbb{P}^{1} \times \mathbb{P}(\mathscr{E}) ; \\
E_{1}^{\prime \prime} \cap E_{W} & =\mathbb{P}^{1} \times \mathbb{P}\left(\mathscr{T}_{\mathbb{P}\left(\mathscr{T}_{X}\right) / X}\right) ; \\
E_{1}^{\prime \prime} \cap \tilde{E}_{i j} & =\mathrm{Bl}_{\Delta}\left(\mathbb{P}\left(\mathscr{T}_{X}\right) \times_{X} \mathbb{P}\left(\mathscr{T}_{X}\right)\right),
\end{aligned}
$$

where $\Delta=\Delta_{\mathbb{P}\left(\mathscr{T}_{X}\right) / X}$ is the diagonal of $\mathbb{P}\left(\mathscr{T}_{X}\right)$ relative to $X$.

Proof. We only need to deal with the intersection with $\tilde{E}_{i j}$. By construction, we have

$$
\tilde{W}_{i j}:=\tilde{W} \cap E_{i j}=\left\{t_{i j}\right\} \times \mathbb{P}\left(\mathscr{T}_{X}\right) \hookrightarrow \tilde{W}=\mathbb{P}^{1} \times \mathbb{P}\left(\mathscr{T}_{X}\right) .
$$

The isomorphism $E_{i j} \cong \mathrm{Bl}_{\Gamma_{\pi}}\left(\mathrm{P}\left(\mathscr{T}_{X}\right) \times X\right)$ gives an exceptional divisor $E_{1, i j}^{\prime} \cong$ $\mathbb{P}\left(\mathscr{T}_{X}\right) \times_{X} \mathbb{P}\left(\mathscr{T}_{X}\right)$. Then $\tilde{W}_{i j}$ is simply the relative diagonal $\Delta$ in the exceptional divisor $E_{1, i j}^{\prime}$. The successive closed immersions $\tilde{W} \subset E_{1, i j}^{\prime} \subset E_{i j}$ gives a short exact sequence

$$
\left.0 \longrightarrow \mathscr{N}_{\tilde{W}_{i j} / E_{1, i j}^{\prime}} \longrightarrow \mathscr{N}_{\tilde{W}_{i j} / E_{i j}} \longrightarrow \mathscr{N}_{E_{1, i j}^{\prime} / E_{i j}}\right|_{\tilde{W}_{i j}} \longrightarrow 0
$$

which is equivalent to (39). Hence we see that

$$
E_{W} \cap \tilde{E}_{i j}=\left\{t_{i j}\right\} \times \mathbb{P}(\mathscr{E}) .
$$

The last identity of the proposition follows from

$$
E_{1}^{\prime \prime} \cap \tilde{E}_{i j}=\mathrm{Bl}_{\tilde{W}_{i j}}\left(E_{1, i j}^{\prime}\right)=\mathrm{Bl}_{\Delta}\left(\mathbb{P}\left(\mathscr{T}_{X}\right) \times_{X} \mathrm{P}\left(\mathscr{T}_{X}\right)\right) .
$$




\section{Multiplicative Chow-Künneth decomposition for $X^{[3]}$}

\subsection{Self-dual $\mathfrak{S}_{3}$-invariant multiplicative Chow-Künneth decomposition} on $\boldsymbol{X}_{\mathbf{3}}$. Let us consider a smooth projective variety $X$ that is equipped with a multiplicative Chow-Künneth decomposition

$$
\Delta_{X}=\pi_{X}^{0}+\pi_{X}^{1}+\cdots+\pi_{X}^{2 d} \text { in } \mathrm{CH}_{d}(X \times X)
$$

that is self-dual, meaning that $\pi_{X}^{2 d-i}$ is the transpose of $\pi_{X}^{i}$ for all $i$. Here $d=$ $\operatorname{dim} X$. Then the product Chow-Künneth decomposition,

$$
\pi_{X^{3}}^{i}:=\sum_{i_{1}+i_{2}+i_{3}=i} \pi_{X}^{i_{1}} \otimes \pi_{X}^{i_{2}} \otimes \pi_{X}^{i_{3}}, \quad 0 \leqslant i \leqslant 6 d
$$

on $X^{3}$ is clearly self-dual (see Proposition 2.4); it is also multiplicative by Proposition 3.2. The symmetric group $\mathfrak{S}_{3}$ acts on $X^{3}$ and the product ChowKünneth decomposition $\left\{\pi_{X^{3}}^{i}\right\}$ is clearly $\mathfrak{S}_{3}$-invariant.

We now take up the notations of Paragraph 5.2 and we assume that $X$ is a smooth projective variety of dimension $d$ equipped with a self-dual multiplicative Chow-Künneth decomposition $\left\{\pi_{X}^{i}\right\}$, with the additional property that the Chern classes $c_{i}(X)$ sit in $\mathrm{CH}^{i}(X)_{0}$ for all $i$. The goal of this paragraph is to show that the variety $X_{3}$ obtained in Paragraph 5.2 by resolving the map $X^{3} \rightarrow X^{[3]}$ is naturally equipped with a self-dual multiplicative Chow-Künneth decomposition that is $\mathfrak{S}_{3}$-invariant.

We define

$$
\mathcal{S}_{0}:=\left\{\Delta_{123}, \Delta_{12}, \Delta_{13}, \Delta_{23}, X_{0}\right\}
$$

and we equip $\Delta_{123} \cong X$ with the Chow-Künneth decomposition $\left\{\pi_{X}^{i}\right\}, \Delta_{i j} \cong X^{2}$ with the product Chow-Künneth decomposition, and $X_{0}=X^{3}$ with the product Chow-Künneth decomposition. These Chow-Künneth decompositions are all self-dual and multiplicative by Proposition 3.2. Moreover, the Chow-Künneth decompositions for $\Delta_{123}$ and $X_{0}$ are $\mathfrak{S}_{3}$-invariant, while the Chow-Künneth decompositions $\left\{\pi_{\Delta_{i j}}^{l}\right\}$ for the big diagonals $\Delta_{i j}$ satisfy $g \cdot \pi_{\Delta_{i j}}^{l}=\pi_{\Delta_{g(i) g(j)}}^{l}$ for all $g \in \mathfrak{S}_{3}$ (here, $g(i)$ is the action of $\mathfrak{S}_{3}$ on the set $\{1,2,3\}$ ).

LEMMA 6.1. The set $\mathcal{S}_{0}$ is complete and admissible in the sense of Definition 4.1.

Proof. That the set $\mathcal{S}_{0}$ is complete is obvious. It is also admissible. Indeed, (a) the Chern classes of $X$ belong to $\mathrm{CH}^{*}(X)_{0}$ by assumption and it is then apparent that the Chern classes of the various normal bundles among the pairs of elements $\left(Y_{j_{1}}\right.$, $Y_{j_{2}}$ ) of $\mathcal{S}_{0}$ such that $Y_{j_{1}} \subset Y_{j_{2}}$ have their Chern classes sitting in $\mathrm{CH}^{*}\left(Y_{j_{1}}\right)_{0}$. Also the multiplicative Chow-Künneth decomposition $\left\{\pi_{X}^{i}\right\}$ is assumed to be self-dual, 
it follows that all possible diagonal inclusions are of pure grade 0 since they are obtained from the graph of the identity morphism. Hence we have (b) the various inclusions $Y_{j_{1}} \subset Y_{j_{2}}$ are of pure grade 0 .

Taking $Y_{0}:=\Delta_{123}$ and denoting $X_{1}=\mathrm{Bl}_{Y_{0}}\left(X_{0}\right)$ the blow-up of $X_{0}$ along $Y_{0}$, one sees that

$$
\mathrm{Bl}_{Y_{0}}\left(\mathcal{S}_{0}\right)=\left\{\tilde{\Delta}_{12}, \tilde{\Delta}_{13}, \tilde{\Delta}_{23}, X_{1}\right\}
$$

Note that the strict transforms of the $\Delta_{i j}: \tilde{\Delta}_{12}, \tilde{\Delta}_{23}$ and $\tilde{\Delta}_{13}$ are pairwise disjoint as subvarieties of $X_{1}$. These are equipped with a self-dual multiplicative ChowKünneth decomposition induced by that of $\Delta_{i j}$ and $\Delta_{123}$ via Proposition 3.4. By Proposition 4.4, the set $\mathrm{Bl}_{Y_{0}}\left(\mathcal{S}_{0}\right)$ is complete and admissible. The self-dual multiplicative Chow-Künneth decompositions of the $\tilde{\Delta}_{i j}$ fit together to give a self-dual multiplicative Chow-Künneth decomposition of $\tilde{\Delta}_{12} \sqcup \tilde{\Delta}_{13} \sqcup \tilde{\Delta}_{23}$ that is $\mathfrak{S}_{3}$-invariant.

Let $E_{1} \subset X_{1}$ be the exceptional divisor of the blow-up $\rho_{1}: X_{1} \rightarrow X_{0}$. In the discussion following (29), we defined a smooth closed subvariety $W \subset E_{1} \subset X_{1}$. Let $W_{i j} \subset \mathbb{P}\left(\mathscr{N}_{Y_{0} / X_{0}}\right)=E_{1}$ be the closed subvarieties obtained in (33) as the geometric projectivizations of the following subbundles $\mathscr{W}_{i j}$ of $\mathscr{N}_{Y_{0} / X_{0}}$

$$
\begin{array}{ll}
\mathscr{W}_{12} \cong \mathscr{T}_{X} \hookrightarrow \mathscr{T}_{X} \oplus \mathscr{T}_{X}, \quad v \mapsto(0, v) ; \\
\mathscr{W}_{23} \cong \mathscr{T}_{X} \hookrightarrow \mathscr{T}_{X} \oplus \mathscr{T}_{X}, \quad v \mapsto(v, 0) ; \\
\mathscr{W}_{13} \cong \mathscr{T}_{X} \hookrightarrow \mathscr{T}_{X} \oplus \mathscr{T}_{X}, \quad v \mapsto(v,-v) .
\end{array}
$$

The isomorphism $\mathscr{W}_{i j} \cong \mathscr{T}_{X}$ gives that the Chern classes $c_{p}\left(\mathscr{W}_{i j}\right)=c_{p}(X)$ sit in $\mathrm{CH}^{p}(X)_{0}$. Hence the subvarieties $W_{i j}$ are naturally endowed with a self-dual multiplicative Chow-Künneth decomposition thanks to Proposition 3.3. Recall that under the isomorphism (32), we have

$$
W_{12} \cong[0: 1] \times \mathbb{P}\left(\mathscr{T}_{X}\right), \quad W_{23} \cong[1: 0] \times \mathbb{P}\left(\mathscr{T}_{X}\right), \quad W_{13} \cong[1:-1] \times \mathbb{P}\left(\mathscr{T}_{X}\right),
$$

and note that scheme-theoretically

$$
W_{i j}=W \cap \tilde{\Delta}_{i j}
$$

We also observe that the variety $W$ is stable under the action of $\mathfrak{S}_{3}$. Let us describe explicitly the action of $\mathfrak{S}_{3}$ on $W$. Note that the isomorphism (30) factors as

$$
E_{1}=\mathbb{P}\left(\frac{\mathscr{T}_{X} \oplus \mathscr{T}_{X} \oplus \mathscr{T}_{X}}{\mathscr{T}_{X}}\right) \cong \mathbb{P}\left(\mathscr{T}_{X} \oplus \mathscr{T}_{X}\right),
$$

where the second isomorphism is induced by the homomorphism $\theta$ in (29). The action of $\mathfrak{S}_{3}$ on $E_{1}$ is given by permuting the three $\mathscr{T}_{X}$-factors of the numerator 
in $\mathbb{P}\left(\mathscr{T}_{X}^{\oplus 3} / \mathscr{T}_{X}\right)$. Under the identification (32), the natural inclusion $W \subset E_{1}$ is given by

$$
\mathbb{P}^{1} \times \mathbb{P}\left(\mathscr{T}_{X}\right) \hookrightarrow \mathbb{P}\left(\mathscr{T}_{X} \oplus \mathscr{T}_{X}\right), \quad([s: t],[v]) \mapsto[s v: t v] .
$$

Then the $\mathfrak{S}_{3}$-action is easy to understand. For example, let $g=(12) \in \mathfrak{S}_{3}$ be the transposition that permutes 1 and 2 . Then we have

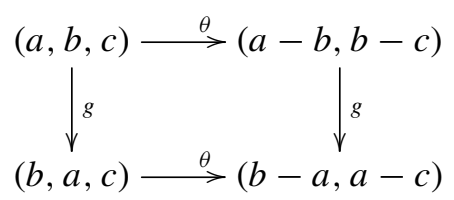

Hence the action of $g$ on $\mathbb{P}\left(\mathscr{T}_{X} \oplus \mathscr{T}_{X}\right)$ is given by $\left[v_{1}: v_{2}\right] \mapsto\left[-v_{1}: v_{1}+v_{2}\right]$. The following diagram is then commutative
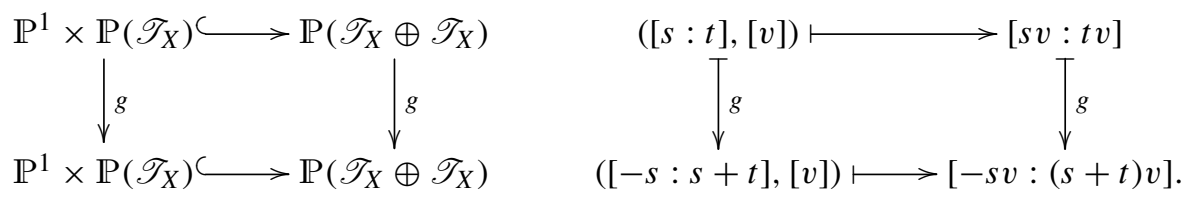

Hence the action of the element $g=(12)$ on $\mathbb{P}^{1} \times \mathbb{P}\left(\mathscr{T}_{X}\right)$ is induced by the action on $\mathbb{P}^{1}$ given by $[s: t] \mapsto[-s: s+t]$. Repeating the above argument for each element of $\mathfrak{S}_{3}$, we conclude that under the isomorphism (32), $W$ is isomorphic to $\mathbb{P}^{1} \times \mathbb{P}\left(\mathscr{T}_{X}\right)$ and this isomorphism is made $\mathfrak{S}_{3}$-equivariant by letting $\mathfrak{S}_{3}$ act trivially on $\mathbb{P}\left(\mathscr{T}_{X}\right)$ and act on $\mathbb{P}^{1}$ as the subgroup of $\operatorname{Aut}\left(\mathbb{P}^{1}\right)$ that permutes $\{[1$ : 0], [1:-1], [0:1]\}. Thus the subvariety $W_{12} \sqcup W_{13} \sqcup W_{23}$ is stable under the action of $\mathfrak{S}_{3}$. Let us endow $\mathbb{P}^{1}$ with the $\mathfrak{S}_{3}$-invariant (for the action described above) self-dual multiplicative Chow-Künneth decomposition $\left\{\pi_{\mathrm{P}^{1}}^{0}:=\{[0: 1]\} \times X\right.$, $\left.\pi_{\mathrm{P}^{1}}^{2}:=X \times\{[0: 1]\}\right\}$ and $\mathbb{P}\left(\mathscr{T}_{X}\right)$ with the $\mathfrak{S}_{3}$-invariant (for the trivial action) multiplicative self-dual Chow-Künneth decomposition given by Proposition 3.3. Then clearly, the obvious Chow-Künneth decomposition on $W$ and $W_{12} \sqcup W_{13} \sqcup$ $W_{23}$ are $\mathfrak{S}_{3}$-invariant, self-dual, and multiplicative.

We define the set $\mathcal{S}_{1}$ of closed subvarieties of $X_{1}$ to be

$$
\mathcal{S}_{1}:=\left\{X_{1}, E_{1}, \tilde{\Delta}_{12} \sqcup \tilde{\Delta}_{13} \sqcup \tilde{\Delta}_{23}, W, W_{12} \sqcup W_{13} \sqcup W_{23}\right\} .
$$

LEMMA 6.2. The set $\mathcal{S}_{1}$ is complete in the sense of Definition 4.1. Moreover, all elements of $\mathcal{S}_{1}$ are stable under the action of $\mathfrak{S}_{3}$ and are endowed with a $\mathfrak{S}_{3}$ invariant self-dual Chow-Künneth decomposition that make $\mathcal{S}_{1}$ admissible. 
Proof. The set $\mathcal{S}_{1}$ is obviously complete and we already saw that all the subvarieties in $\mathcal{S}_{1}$ are smooth and endowed with a $\mathfrak{S}_{3}$-invariant self-dual multiplicative Chow-Künneth decomposition. Hence it remains to verify condition (ii) in the definition of an admissible set (Definition 4.1). This condition involves the normal bundles and all possible inclusions of varieties in $\mathcal{S}_{1}$. The inclusions $W_{i j} \hookrightarrow W$ are taken care of by the isomorphisms (32) and (41). For the inclusion of $W_{i j} \hookrightarrow \tilde{\Delta}_{i j}$, we only need to note that, under the isomorphism $\tilde{\Delta}_{i j} \cong \mathrm{Bl}_{\Delta_{X}}(X \times X)$, the subvariety

$$
W_{i j} \cong \mathbb{P}\left(\mathscr{T}_{X}\right) \hookrightarrow \mathrm{Bl}_{\Delta_{X}}(X \times X)
$$

is simply the exceptional divisor. The inclusion $E_{1} \subset X_{1}$ is also the exceptional divisor. Both of the above two cases are taken care of by Proposition 3.4 and Remark 3.6. It remains to deal with the inclusion $W \subset E_{1}$. Note that the normal bundle of $W \subset E_{1}$ is given by (35). Let $\iota: W \hookrightarrow E_{1}$ be the inclusion. Let $\xi \in \mathrm{CH}^{1}\left(E_{1}\right)$ be the Chern class of $\mathcal{O}_{E_{1}}(1)$ and let $\xi_{1}=p_{1}^{*} c_{1}\left(\mathcal{O}_{\mathbb{P}^{1}}(1)\right) \in \mathrm{CH}^{1}(W)$ and $\xi_{2}=p_{2}^{*} c_{1}\left(\mathcal{O}_{\mathbb{P}\left(\mathscr{T}_{X}\right)}(1)\right) \in \mathrm{CH}^{1}(W)$ be the pull-backs of the corresponding Chern classes. Equation (34) implies

$$
\iota^{*} \xi=\xi_{1}+\xi_{2}
$$

and hence it follows that $\iota^{*}$ is compatible with the gradings on the Chow groups. On $E_{1} \cong \mathbb{P}\left(\mathscr{T}_{X} \oplus \mathscr{T}_{X}\right)$, the natural homomorphism $\mathcal{O}_{E_{1}}(-1) \subset \pi_{2}^{*}\left(\mathscr{T}_{X} \oplus \mathscr{T}_{X}\right)$ gives rise to two homomorphisms,

$$
\varphi_{1}: \mathcal{O}_{E_{1}}(-1) \hookrightarrow \pi_{2}^{*} \mathscr{T}_{X} \quad \text { and } \quad \varphi_{2}: \mathcal{O}_{E_{1}}(-1) \hookrightarrow \pi_{2}^{*} \mathscr{T}_{X},
$$

by composing the two projections. Here $\pi_{2}: \mathbb{P}\left(\mathscr{T}_{X} \oplus \mathscr{T}_{X}\right) \rightarrow X$ is the structure morphism. Let $\mathscr{Q}_{1}$ be the quotient of $\varphi_{1}$. Then $\varphi_{2}$ induces a homomorphism $\mathcal{O}_{E_{1}}(-1) \rightarrow \mathscr{Q}_{1}$. This can be viewed as a section $s$ of the sheaf $\mathscr{Q}_{1} \otimes \mathcal{O}_{E_{1}}(1)$ whose vanishing locus is exactly $W$. It follows that

$$
\iota_{*}[W]=c_{d-1}\left(\mathscr{Q}_{1} \otimes \mathcal{O}_{E_{1}}(1)\right) \in \mathrm{CH}^{d-1}\left(E_{1}\right)_{0} .
$$

It follows that

$$
\iota_{*}\left(\xi_{2}^{l}+l \xi_{2}^{l-1} \xi_{1}\right)=\iota_{*}\left(\xi_{1}+\xi_{2}\right)^{l}=\iota_{*} \iota^{*} \xi^{l}=\iota_{*}[W] \cdot \xi^{l} \in \mathrm{CH}^{*}\left(E_{1}\right)_{0} .
$$

We also note that Lemma 4.7 implies that $\iota_{*}\left(\xi_{1} \xi_{2}^{l-1}\right) \in \mathrm{CH}^{*}\left(E_{1}\right)_{0}$. It follows that

$$
\iota_{*}\left(\xi_{1}^{a} \xi_{2}^{b}\right) \in \mathrm{CH}^{*}\left(E_{1}\right)_{0}, \quad \text { for all } a, b \geqslant 0 .
$$

Thus one easily concludes that $\iota_{*}$ respects the gradings on the Chow groups. The above argument also works for the inclusion

$$
1 \times \imath: Z \times W \longrightarrow Z \times E_{1},
$$


where $Z$ is an arbitrary smooth projective variety with a self-dual multiplicative Chow-Künneth decomposition. We take $Z$ to be $W$. Then the graph of $\iota$ is the push-forward of $\Delta_{W}$ via the morphism $1 \times \iota$. Hence $\iota$ is of pure grade 0 . By Remark 4.2, this proves that $\mathcal{S}_{1}$ is admissible.

Let now (as in paragraph 5.2) $X_{2}:=\mathrm{Bl}_{Y_{1}}\left(X_{1}\right)$, where $Y_{1}:=\tilde{\Delta}_{12} \sqcup \tilde{\Delta}_{13} \sqcup \tilde{\Delta}_{23}$. By Proposition 4.4, we see that the set

$$
\mathcal{S}_{2}=\left\{\tilde{W}, E_{1}^{\prime}, X_{2}\right\}
$$

where $\tilde{W}$ is the strict transform of $W$, is admissible. Moreover, $\tilde{W}$ is equipped with a $\mathfrak{S}_{3}$-invariant self-dual multiplicative Chow-Künneth decomposition.

Let finally $X_{3}$ be the blow-up of $X_{2}$ along $Y_{2}:=\tilde{W}$. We then have

PROPOSITION 6.3. The variety $X_{3}$ admits $a \mathfrak{S}_{3}$-invariant self-dual multiplicative Chow-Künneth decomposition.

Proof. The proposition follows immediately from the discussion above and from Proposition 3.4 applied to $X_{2}$ blown up along $Y_{2}$.

6.2. Proof of Theorem 1. In Section 5, we resolved the map $X^{3}-\rightarrow X^{[3]}$ into a morphism $p: X_{3} \rightarrow X^{[3]}$. The smooth projective variety $X_{3}$ is naturally equipped with a $\mathfrak{S}_{3}$-action, and the morphism $p$ is generically the quotient morphism, meaning that there exists a $\mathfrak{S}_{3}$-invariant open subset $U \subset X_{3}$ such $\left.p\right|_{U}$ is the quotient morphism. Proposition 6.3 shows that $X_{3}$ is naturally endowed with a $\mathfrak{S}_{3}$ invariant self-dual multiplicative Chow-Künneth decomposition, whenever $X$ is endowed with a self-dual multiplicative Chow-Künneth decomposition with the additional property that $c_{p}(X)$ sit in $\mathrm{CH}^{p}(X)_{0}$ for all $p$. The following technical lemma gives sufficient geometric conditions on the locus that is contracted by a generic quotient (for the action of a finite group $G$ ) morphism $p$ for a $G$-invariant self-dual multiplicative Chow-Künneth decomposition to descend along $p$.

LEMMA 6.4. Let $G$ be a finite group and let $X$ be a smooth projective variety with $a G$-action. Assume that $X$ is endowed with a $G$-invariant self-dual multiplicative Chow-Künneth decomposition $\left\{\pi_{X}^{i}\right\}_{i=0}^{2 d}$, where $d=\operatorname{dim} X$. Let $p: X \rightarrow Y$ be a generically finite morphism such that the following conditions hold.

(a) The morphism $p$ is generically the quotient morphism of the G-action. Namely, there exists a G-invariant open subset $U$ of $X$ such that $\left.p\right|_{U}$ is the quotient morphism $U \rightarrow U / G$. 
(b) The morphism $p$ contracts two smooth divisors $D_{i} \subset X$, $i=1$, 2. Let $Y_{i} \subset Y$ be the image of $D_{i}$. Each $D_{i}$ is equipped with a multiplicative Chow-Künneth decomposition which is compatible with that of $X$. (This means that the inclusion morphisms are of pure grade 0 .)

(c) All components of $D_{2} \times_{Y_{2}} D_{2}$ are of dimension $d$ and viewed as cycles on $X \times X$, they are all in $\mathrm{CH}^{d}(X \times X)_{0}$.

(d) $Y_{1}$ is smooth and all the Chern classes of $\left(\left.p\right|_{D_{1}}\right)^{*} \mathscr{T}_{Y_{1}}$ are in $\mathrm{CH}^{*}\left(D_{1}\right)_{0}$; the Chern classes of $p^{*} \mathscr{T}_{Y}$ sit in $\mathrm{CH}^{*}(X)_{0}$.

(e) $Y_{1}$ is disjoint from $p\left(D_{2} \backslash D_{1}\right)$.

(f) The morphism $\left.p\right|_{D_{1}}: D_{1} \rightarrow Y_{1}$ is smooth and $D_{1} \times_{Y_{1}} D_{1}$, viewed as a cycle on $D_{1} \times D_{1}$, is in $\mathrm{CH}^{*}\left(D_{1} \times D_{1}\right)_{0}$.

Then ${ }^{t} \Gamma_{p} \circ \Gamma_{p}$ sits in $\mathrm{CH}^{d}(X \times X)_{0}$. Furthermore, $Y$ admits a multiplicative ChowKünneth decomposition such that the Chern classes of $Y$ sit in $\mathrm{CH}^{*}(Y)_{0}$.

Proof. Note that we have the following fiber square

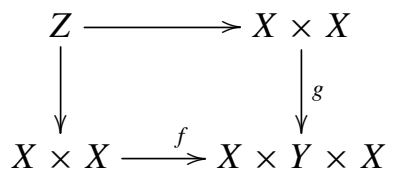

where $f\left(x_{1}, x_{2}\right)=\left(x_{1}, p\left(x_{1}\right), x_{2}\right)$ and $g\left(x_{1}, x_{2}\right)=\left(x_{1}, p\left(x_{2}\right), x_{2}\right)$. By definition ${ }^{t} \Gamma_{p} \circ \Gamma_{p}$ is supported on $Z$. A component of $Z$ is called dominating if it dominates $X$ via any of the two projections. A general point on a dominating component is of the form $\left(x_{1}, x_{2}\right)$ such that $p\left(x_{1}\right)=p\left(x_{2}\right)$ is a general point on $Y$. The assumption (a) implies that $x_{1}$ and $x_{2}$ are in the same orbit. Hence any dominating component of $Z$ must be the graph of an element of $G$. Since the multiplicative ChowKünneth decomposition on $X$ is $G$-invariant, we see that a dominating component of $Z$ is in $\mathrm{CH}^{d}(X \times X)_{0}$. The components of $Z$ that are contained in $D_{2} \times_{Y_{2}} D_{2}$ are taken care of by assumption (c). By (e), all the remaining components of $Z$ are contained in $D_{1} \times_{Y_{1}} D_{1}$. We use the excess intersection formula [6, Section 6.3, Theorem 6.3] to deal with the remaining components. To do that let us fix some notations. Let $\mathscr{N}_{p}$ be the normal bundle of the graph of $p$ inside $X \times Y$. We then have the following short exact sequence of sheaves on $X$.

$$
0 \longrightarrow \mathscr{T}_{X} \stackrel{\text { (id,d } \mathrm{d} p)}{\longrightarrow} \mathscr{T}_{X} \oplus p^{*} \mathscr{T}_{Y} \longrightarrow \mathscr{N}_{p} \longrightarrow 0 .
$$


It follows that $c\left(\mathscr{N}_{p}\right)=c\left(p^{*} \mathscr{T}_{Y}\right)$. Hence, by $(\mathrm{d})$,

$$
c\left(\mathscr{N}_{p}\right) \in \mathrm{CH}^{*}(X)_{0} .
$$

We need to understand the normal bundle $\mathscr{N}_{1}$ of $D_{1} \times_{Y_{1}} D_{1}$ inside $X \times X$. Consider the successive inclusion

$$
D_{1} \times_{Y_{1}} D_{1} \stackrel{j_{1}}{\longrightarrow} D_{1} \times D_{1} \stackrel{j_{2}}{\longrightarrow} X \times X .
$$

The assumption (f) implies that the normal bundle associated to the first inclusion is $f^{*} \mathscr{T}_{Y_{1}}$, where $f: D_{1} \times_{Y_{1}} D_{1} \rightarrow Y_{1}$ is the natural morphism; the normal bundle associated to the second inclusion is $p_{1}^{*} \mathscr{N}_{D_{1} / X} \oplus p_{2}^{*} \mathscr{N}_{D_{1} / X}$, where $p_{1}$ and $p_{2}$ are the two projections of $D_{1} \times D_{1}$. Hence $\mathscr{N}_{1}$ fits into the following short exact sequence

$$
0 \longrightarrow f^{*} \mathscr{T}_{Y_{1}} \longrightarrow \mathscr{N}_{1} \longrightarrow p_{1}^{*} \mathscr{N}_{D_{1} / X} \oplus p_{2}^{*} \mathscr{N}_{D_{1} / X} \longrightarrow 0
$$

where the last term is viewed as its restriction to $D_{1} \times_{Y_{1}} D_{1}$. By [6, Section 6.3, Theorem 6.3], the contribution to ${ }^{t} \Gamma_{p} \circ \Gamma_{p}$ which comes from the component $D_{1} \times_{Y_{1}} D_{1}$ of $Z$ is given by

$$
j_{*} c_{\text {top }}\left(j^{*} q_{1}^{*} \mathscr{N}_{p} / \mathscr{N}_{1}\right)
$$

Here, $j=j_{2} \circ j_{1}: D_{1} \times_{Y_{1}} D_{1} \rightarrow X \times X$ is the inclusion and $q_{1}: X \times X \rightarrow X$ is the projection to the first factor. To show that $j_{*} c_{\text {top }}\left(j^{*} q_{1}^{*} \mathscr{N}_{p} / \mathscr{N}_{1}\right)$ lies in $\mathrm{CH}^{*}(X \times X)_{0}$, it suffices to check that

$$
j_{*} c\left(\mathscr{N}_{1}\right) \in \mathrm{CH}^{*}(X \times X)_{0} .
$$

Thanks to the short exact sequence (42), this is further reduced to showing that

$$
j_{*} c\left(f^{*} \mathscr{T}_{Y_{1}}\right) \in \mathrm{CH}^{*}(X \times X)_{0} .
$$

Note that $f^{*} \mathscr{T}_{Y_{1}}=j_{1}^{*} p_{1}^{*}\left(\left.p\right|_{D_{1}}\right)^{*} \mathscr{T}_{Y_{1}}$ and hence

$$
j_{*} c\left(f^{*} \mathscr{T}_{Y_{1}}\right)=j_{2, *} j_{1, *} j_{1}^{*} p_{1}^{*} c\left(\left(\left.p\right|_{D_{1}}\right)^{*} \mathscr{T}_{Y_{1}}\right)=j_{2, *}\left(\left[D_{1} \times_{Y_{1}} D_{1}\right] \cdot p_{1}^{*} c\left(\left(\left.p\right|_{D_{1}}\right)^{*} \mathscr{T}_{Y_{1}}\right)\right) .
$$

It follows from the first part of (d), the second part of (f) and the compatibility of $j_{2, *}$ with the grading of the Chow rings that $j_{*} c\left(f^{*} \mathscr{T}_{Y_{1}}\right) \in \mathrm{CH}^{*}(X \times X)_{0}$.

The conclusion concerning the Chern classes of $Y$ being in the graded-0 part follows immediately from the second part of (d) and the explicit Chow-Künneth decomposition on $Y$ as is given by Proposition 2.12.

We are finally in a position to prove Theorem 1, which we restate here for the convenience of the reader. 
THEOREM 6.5 (Theorem 1). Let $X$ be a smooth projective variety that admits a self-dual multiplicative Chow-Künneth decomposition $\left\{\pi_{X}^{i}\right\}$. Let us denote as always $\mathrm{CH}^{i}(X)_{s}:=\left(\pi_{X}^{2 i-s}\right)_{*} \mathrm{CH}^{i}(X)$. Assume that the Chern classes $c_{p}(X)$ of $X$ belong to $\mathrm{CH}^{p}(X)_{0}$. Then the Hilbert cube $X^{[3]}$ admits a self-dual multiplicative Chow-Künneth decomposition, with the property that the Chern classes $c_{p}\left(X^{[3]}\right)$ sit in the degree-zero graded pieces $\mathrm{CH}^{p}\left(X^{[3]}\right)_{0}$.

Proof. In Proposition 5.1, we obtain a generically finite quotient morphism $p: X_{3} \rightarrow X^{[3]}$ by the symmetric group $\mathfrak{S}_{3}$. By Proposition $6.3, X_{3}$ has a $\mathfrak{S}_{3}-$ invariant self-dual multiplicative Chow-Künneth decomposition. It then follows from Proposition 3.7 that $X^{[3]}$ is endowed with a self-dual multiplicative ChowKünneth decomposition as long as ${ }^{t} \Gamma_{p} \circ \Gamma_{p}$ belongs to $\mathrm{CH}^{d}\left(X_{3} \times X_{3}\right)_{0}$. To achieve this, we recall from Proposition 5.2 that the morphism $p$ contracts a divisor $E_{1}^{\prime \prime} \subset X_{3}$ to $B_{3} \subset X^{[3]}$ and another divisor $E_{W}$ to $B_{2} \subset X^{[3]}$. Here $B_{2}$ and $B_{3}$ form the stratification of Section 5.1. $E_{W}$ is the exceptional divisor of the blowup $X_{3} \rightarrow X_{2}$, and $E_{1}^{\prime \prime}$ is the strict transform of $E_{1}^{\prime} \subset X_{2}$; see Section 5.2 for the notation. We would like to apply Lemma 6.4 to $p: X_{3} \rightarrow X^{[3]}$ with $D_{1}=E_{1}^{\prime \prime}$, $D_{2}=E_{W}, Y_{1}=B_{3}$ and $Y_{2}=B_{2}$. We need to verify all the assumptions of Lemma 6.4. For simplicity, we write $Y=X^{[3]}$.

Assumption (a). This is immediate from the construction of $p$ as the desingularization of $X^{3} \rightarrow X^{(3)}$.

Assumption (b). We need to see that $D_{1}=E_{1}^{\prime \prime}$ and $D_{2}=E_{W}$ have multiplicative Chow-Künneth decompositions that are compatible with that of $X_{3}$. The case of $D_{2}$ follows from the fact that it is the exceptional divisor of the last blow-up $X_{3} \rightarrow$ $X_{2}$; see Remark 3.6. The case of $D_{1}$ can be done as follows. By the discussion following Lemma 6.2, we have a complete admissible set $\mathcal{S}_{2}$ of subvarieties of $X_{2}$. Hence we get that

$$
\mathcal{S}_{3}=\mathrm{Bl}_{Y_{2}}\left(\mathcal{S}_{2}\right)=\left\{E_{1}^{\prime \prime}, X_{3}\right\}
$$

is admissible (see Proposition 4.4) and, in particular, the inclusion $D_{1} \hookrightarrow X_{2}$ is of pure grade 0 .

Assumption (c). We have the isomorphism $D_{2} \cong \mathbb{P}^{1} \times \mathbb{P}(\mathscr{E})$; see (40). Under this isomorphism, the grading of the Chow ring of $D_{2}$ and $D_{2} \times D_{2}$ are given by

$$
\begin{aligned}
\mathrm{CH}^{*}\left(D_{2}\right)_{s} & =\sum_{a, b} \xi^{a} \zeta^{b} \cdot \mathrm{CH}^{*}(X)_{s} ; \\
\mathrm{CH}^{*}\left(D_{2} \times D_{2}\right)_{s} & =\sum_{a_{1}, a_{2}, b_{1}, b_{2}} \xi_{1}^{a_{1}} \xi_{2}^{a_{2}} \zeta_{1}^{b_{1}} \zeta_{2}^{b_{2}} \cdot \mathrm{CH}^{*}(X \times X)_{s},
\end{aligned}
$$

where $\xi$ is the Chern class of the $\mathcal{O}(1)$-bundle on $\mathrm{P}(\mathscr{E})$ and $\zeta$ is the Chern class of the $\mathcal{O}(1)$-bundle on $\mathbb{P}\left(\mathscr{T}_{X}\right)$ pulled back to $\mathbb{P}(\mathscr{E})$. The morphism $D_{2} \rightarrow Y_{2}$ can 
be decomposed as the projection $\mathbb{P}^{1} \times \mathbb{P}(\mathscr{E}) \longrightarrow \mathbb{P}(\mathscr{E})$ followed by a morphism $\mathbb{P}(\mathscr{E}) \rightarrow B_{2}$ contracting $\mathbb{P}\left(\mathscr{T}_{\mathbb{P}\left(\mathscr{T}_{X}\right) / X}\right)$ to $B_{3}$. It follows that $D_{2} \times_{Y_{2}} D_{2}$ consists of two components. Then one component of $D_{2} \times_{Y_{2}} D_{2}$ is $\mathbb{P}^{1} \times \mathbb{P}^{1} \times \mathbb{P}(\mathscr{E})$ which lies in $\mathrm{CH}_{3 d}\left(D_{2} \times D_{2}\right)_{0}$. The second component of $D_{2} \times_{Y_{2}} D_{2}$ sits above $Y_{1}=B_{3} \cong$ $\mathrm{G}\left(2, \mathscr{T}_{X}\right)$, which is easily seen to be $\mathbb{P}^{1} \times \mathbb{P}^{1} \times \mathbb{P}\left(\mathscr{T}_{\mathbb{P}\left(\mathscr{T}_{X}\right) / X}\right) \times_{B_{3}} \mathbb{P}\left(\mathscr{T}_{\mathbb{P}\left(\mathscr{T}_{X}\right) / X}\right)$. We note that there is a canonical isomorphism

$$
\mathbb{P}\left(\mathscr{T}_{\mathbb{P}\left(\mathscr{T}_{X}\right) / X}\right) \mathrm{G}\left(1,2, \mathscr{T}_{X}\right)
$$

where $\mathrm{G}\left(1,2, \mathscr{T}_{X}\right)$ is the flag variety bundle over $X$. Under this isomorphism, the morphism $\mathbb{P}\left(\mathscr{T}_{\mathbb{P}\left(\mathscr{T}_{X}\right) / X}\right) \rightarrow B_{3}$ becomes the natural projection $\mathrm{G}\left(1,2, \mathscr{T}_{X}\right) \rightarrow$ $\mathrm{G}\left(2, \mathscr{T}_{X}\right)$. Then the second component becomes

$$
\mathrm{P}^{1} \times \mathbb{P}^{1} \times \mathrm{G}\left(1,2, \mathscr{T}_{X}\right) \times_{\mathrm{G}\left(2, \mathscr{T}_{X}\right)} \mathrm{G}\left(1,2, \mathscr{T}_{X}\right)
$$

which can again been directly checked to be in $\mathrm{CH}_{3 d}\left(D_{2} \times D_{2}\right)_{0}$, thanks to the equation (43). Since the inclusion $D_{2} \hookrightarrow X_{3}$ respects the gradings, we see that all components of $D_{2} \times_{Y_{2}} D_{2}$ are contained in $\mathrm{CH}_{3 d}\left(X_{3} \times X_{3}\right)_{0}$.

Assumption (e). We have $p\left(Y_{2} \backslash Y_{1}\right)=B_{2} \backslash B_{3}$.

Assumptions $(d)$ and $(f)$. We already saw that $Y_{1} \cong \mathrm{G}\left(2, \mathscr{T}_{X}\right)$ and hence $Y_{1}$ is smooth. Now we give an explicit description of the morphism $D_{1}=E_{1}^{\prime \prime} \rightarrow Y_{1}=$ $\mathrm{G}\left(2, \mathscr{T}_{X}\right)$. Consider the following diagram

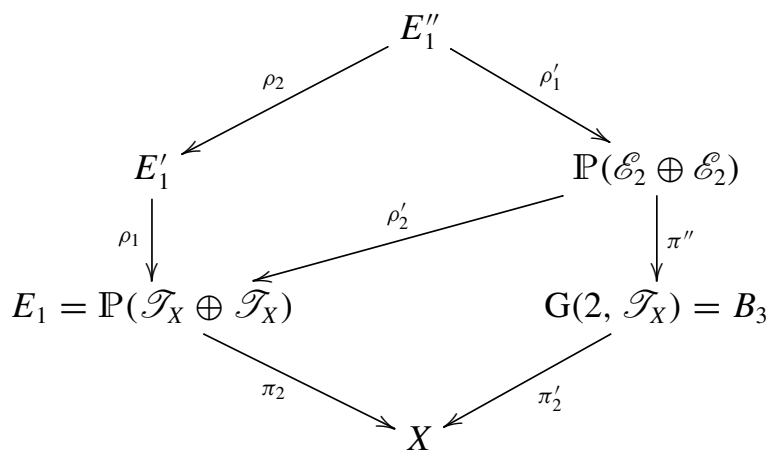

Here $\mathscr{E}_{2}$ is the natural rank-2 subbundle of $\left.\mathscr{T}_{X}\right|_{\mathrm{G}\left(2, \mathscr{T}_{X}\right)}$ on $\mathrm{G}\left(2, \mathscr{T}_{X}\right)$. The morphism $\rho_{2}^{\prime}$ is the blow-up of $E_{1}$ along $W$ and $\rho_{1}^{\prime}$ is the blow-up of $\mathbb{P}\left(\mathscr{E}_{2} \oplus \mathscr{E}_{2}\right)$ along the union of $\tilde{W}_{i j}$, where

$$
\begin{aligned}
\tilde{W}_{12}=\mathbb{P}\left(\mathscr{E}_{2}\right) \hookrightarrow \mathbb{P}\left(\mathscr{E}_{2} \oplus \mathscr{E}_{2}\right), & & {[v] \mapsto[0, v] ; } \\
\tilde{W}_{23}=\mathbb{P}\left(\mathscr{E}_{2}\right) \hookrightarrow \mathbb{P}\left(\mathscr{E}_{2} \oplus \mathscr{E}_{2}\right), & & {[v] \mapsto[v, 0] ; } \\
\tilde{W}_{13}=\mathbb{P}\left(\mathscr{E}_{2}\right) \hookrightarrow \mathbb{P}\left(\mathscr{E}_{2} \oplus \mathscr{E}_{2}\right), & & {[v] \mapsto[v,-v] . }
\end{aligned}
$$


Furthermore, we have $\left.p\right|_{E_{1}^{\prime \prime}}=\pi^{\prime \prime} \circ \rho_{1}^{\prime}$. One uses this description and Lemma 4.5 to study the relative tangent bundle $\mathscr{T}_{E_{1}^{\prime \prime} / B_{3}}$ (and hence also $\left.\left(\left.p\right|_{E_{1}^{\prime \prime}}\right)^{*} \mathscr{T}_{B_{3}}\right)$ and shows that their Chern classes are in $\mathrm{CH}^{*}\left(E_{1}^{\prime \prime}\right)_{0}$. The smoothness of $\left.p\right|_{E_{1}^{\prime \prime}}$ can be checked by showing that the fibers are all smooth. Now we want to show that $E_{1}^{\prime \prime} \times_{B_{3}}$ $E_{1}^{\prime \prime}$ is in $\mathrm{CH}^{*}\left(E_{1}^{\prime \prime} \times E_{1}^{\prime \prime}\right)_{0}$. This can be done by a Chern class computation. The multiplicative Chow-Künneth decomposition on $E_{1}^{\prime \prime}$ is constructed from that of $X$ along the morphisms $\pi_{2}, \rho_{1}$ and $\rho_{2}$. The induced grading on the Chow ring of $E_{1}^{\prime \prime}$ can be explicitly described using the canonical cycles appearing in each step. By a 'change of basis' computation, those cycles can be replaced by those appearing in the construction of $\pi_{2}^{\prime}, \pi^{\prime \prime}$ and $\rho_{1}^{\prime}$. More precisely, we have

$$
\mathrm{CH}^{*}\left(E_{1}^{\prime \prime} \times E_{1}^{\prime \prime}\right)_{s}=\mathrm{CH}^{*}(X \times X)_{s}\left[c .\left(\mathscr{E}_{2}\right)_{1}, c .\left(\mathscr{E}_{2}\right)_{2}, \xi_{1}^{\prime}, \xi_{2}^{\prime}, \tau_{i j, 1}, \tau_{i j, 2}\right],
$$

where the right-hand side means polynomials in the given variables with coefficients in $\mathrm{CH}^{*}(X \times S)_{s}$. Here $\xi^{\prime}$ is the $\mathcal{O}(1)$-class on $\mathrm{P}\left(\mathscr{E}_{2} \oplus \mathscr{E}_{2}\right)$ and $\tau_{i j}$ are the classes of the exceptional divisors of the blow-up $\rho_{1}^{\prime}$; the subscripts ' 1 ' and ' 2 ' indicate which factor they come from. Let $f: E_{1}^{\prime \prime} \rightarrow X$ be the natural morphism, then

$$
E_{1}^{\prime \prime} \times_{B_{3}} E_{1}^{\prime \prime} \subset(f \times f)^{-1}\left(\Delta_{X}\right)
$$

is the vanishing locus of the morphism

$$
p_{1}^{*} \mathscr{E}_{2} \rightarrow \frac{\mathscr{T}_{X}}{p_{2}^{*} \mathscr{E}_{2}} .
$$

Given the formula (45), a Chern class computation shows that $E_{1}^{\prime \prime} \times_{B_{3}} E_{1}^{\prime \prime} \in$ $\mathrm{CH}^{*}\left(E_{1}^{\prime \prime} \times E_{1}^{\prime \prime}\right)_{0}$.

Now it only remains to show that the Chern classes of $p^{*} \mathscr{T}_{Y}$ are contained in $\mathrm{CH}^{*}(X)_{0}$. We consider the short exact sequence

$$
0 \longrightarrow p^{*} \Omega_{Y}^{1} \longrightarrow \Omega_{X_{3}}^{1} \longrightarrow \Omega_{X_{3} / Y}^{1} \longrightarrow 0 .
$$

Since $c\left(\mathscr{T}_{X}\right) \in \mathrm{CH}^{*}\left(X_{3}\right)_{0}$, we only need to show that the Chern classes of $\Omega_{X_{3} / Y}^{1}$ are in $\mathrm{CH}^{*}\left(X_{3}\right)_{0}$. Note that $\Omega_{X_{3} / Y}^{1}$ restricted to $\tilde{E}_{i j}$ is simply the conormal bundle of $\tilde{E}_{i j}$ in $X_{3}$. From the short exact sequence

$$
\left.\left.0 \longrightarrow \Omega_{X_{3} / Y}^{1}\right|_{E_{1}^{\prime \prime} \cup E_{W}} \otimes \mathcal{O}\left(-\sum \tilde{E}_{i j}\right) \longrightarrow \Omega_{X_{3} / Y}^{1} \longrightarrow \Omega_{X_{3} / Y}^{1}\right|_{\cup \tilde{E}_{i j}} \longrightarrow 0
$$

we see that it suffices to show that the Chern classes of $\left.\Omega_{X_{3} / Y}^{1}\right|_{E_{1}^{\prime \prime} \cup E_{W}}$ pushes forward into $\mathrm{CH}^{*}\left(X_{3}\right)_{0}$. Then we can again use a similar short exact sequence

$$
\left.\left.\left.0 \longrightarrow \Omega_{X_{3} / Y}^{1}\right|_{E_{W}} \otimes \mathcal{O}_{E_{W}}\left(-E_{1}^{\prime \prime} \cap E_{W}\right) \longrightarrow \Omega_{X_{3} / Y}^{1}\right|_{E_{1}^{\prime \prime} \cup E_{W}} \longrightarrow \Omega_{X_{3} / Y}^{1}\right|_{E_{1}^{\prime \prime}} \longrightarrow 0
$$


to reduce to showing that the Chern classes of $\left.\Omega_{X_{3} / Y}^{1}\right|_{E_{1}^{\prime \prime}}$ and $\left.\Omega_{X_{3} / Y}^{1}\right|_{E_{W}}$ are in the graded-0 part. But then this becomes immediate since these two sheaves are simply $\Omega_{E_{1}^{\prime \prime} / B_{3}}^{1}$ and $\Omega_{E_{W} / B_{2}}^{1}$, respectively.

Thus we verified all the assumptions of Lemma 6.4 and the main theorem follows.

\section{Multiplicative Chow-Künneth decomposition for $X^{[1,2]}$ and $X^{[2,3]}$}

Let $X$ be a projective variety. Recall that, for positive integers $n<m$, the nested Hilbert scheme $X^{[n, m]}$ is the projective variety consisting of $\{(x, y): x \subset y\} \subset$ $X^{[n]} \times X^{[m]}$. If $X$ is smooth and has dimension $\geqslant 3$, Cheah [4] showed that the nested Hilbert scheme $X^{[n, m]}$ is smooth if and only if it is one of $X^{[1,2]}$ or $X^{[2,3]}$.

THEOREM 7.1. Let $X$ be a smooth projective variety that admits a self-dual multiplicative Chow-Künneth decomposition. Assume that the Chern classes of $X$ satisfy $c_{p}(X) \in \mathrm{CH}^{p}(X)_{0}$. Then the nested Hilbert schemes $X^{[1,2]}$ and $X^{[2,3]}$ also admit a self-dual multiplicative Chow-Künneth decomposition, with the property that the Chern classes $c_{p}\left(X^{[1,2]}\right)$ sit in $\mathrm{CH}^{p}\left(X^{[1,2]}\right)_{0}$, respectively $c_{p}\left(X^{[2,3]}\right)$ sit in $\mathrm{CH}^{p}\left(X^{[2,3]}\right)_{0}$.

Proof. The nested Hilbert scheme $X^{[1,2]}$ is by definition the same as the universal length-2 closed subscheme over $X^{[2]}$, which is isomorphic to the blow-up of $X \times X$ along the diagonal. The latter was already treated in [12, Theorem 6].

The nested Hilbert scheme $X^{[2,3]}$ is the blow-up of $X \times X^{[2]}$ along the universal length-2 subscheme. The latter, which we denote $Y$, is isomorphic to $X^{[1,2]}$. By [12, Theorem 6] and the above, both $X \times X^{[2]}$ and $Y \simeq X^{[1,2]}$ admit a self-dual multiplicative Chow-Künneth decomposition such that their Chern classes are of pure grade 0. Therefore, by Proposition 3.4, we only need to check that the set $\left\{Y, X \times X^{[2]}\right\}$ is admissible in the sense of Definition 4.1, that is, we only need to check that

(i) the Chern classes of the normal bundle $\mathscr{N}_{Y / X \times X^{[2]}}$ sit in $\mathrm{CH}^{*}(Y)_{0}$;

(ii) the morphism $i: Y \rightarrow X \times X^{[2]}$ is of pure grade 0 .

We first show (i). As already observed, $Y$ is the blow-up of $X \times X$ along the diagonal and is naturally equipped with a self-dual multiplicative Chow-Künneth decomposition. Let $\rho: Y \rightarrow X \times X$ be the blow-up morphism. Then $\rho$ is of pure 
grade 0 by Proposition 3.4. Consider the following diagram

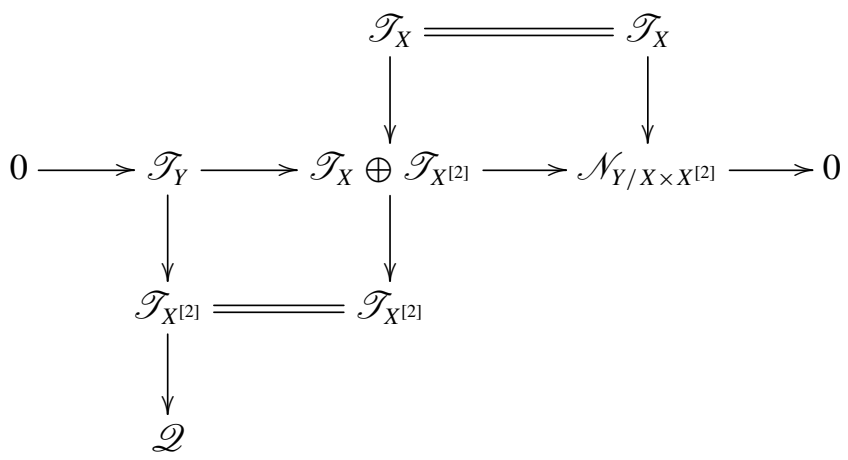

where all sheaves are understood to be their pull-back to $Y$ and $\mathscr{Q}$ is the quotient of $\left.\mathscr{T}_{X^{[2]}}\right|_{Y}$ by $\mathscr{T}_{Y}$. We already know that all the Chern classes of $\mathscr{Q}$ are in the graded-0 part $\mathrm{CH}^{*}(Y)$. Note that every local section $v$ of $\mathscr{N}_{Y / X \times X^{[2]}}$ lifts to $\left(v_{1}\right.$, $\left.v_{2}\right) \in \mathscr{T}_{X^{[2]}} \oplus \mathscr{T}_{X}$, and that the image $\bar{v}_{2} \in \mathscr{Q}$ is independent of the choice of the lifting $\left(v_{1}, v_{2}\right)$. This gives a homomorphism $\mathscr{N}_{Y / X \times X^{[2]}} \longrightarrow \mathscr{Q}$, which fits into the following short exact sequence

$$
\left.0 \longrightarrow \mathscr{T}_{X}\right|_{Y} \longrightarrow \mathscr{N}_{Y / X \times X^{[2]}} \longrightarrow \mathscr{Q} \longrightarrow 0
$$

By assumption, the Chern classes of $\mathscr{T}_{X}$ are in $\mathrm{CH}^{*}(X)_{0}$. Moreover, the natural morphism $Y \hookrightarrow X \times X^{[2]} \rightarrow X$ is the composition of $\rho$ with a projection $X \times X \rightarrow$ $X$, the two of which are of pure grade 0. It follows that the Chern classes of $\left.\mathscr{T}_{X}\right|_{Y}$ sit in $\mathrm{CH}^{*}(Y)_{0}$. The multiplicativity of $\mathrm{CH}^{*}(Y)_{0}$ then implies that the Chern classes of $\mathscr{N}_{Y / X \times X^{[2]}}$ are in the graded-0 part.

Now we show (ii). To show that the graph $\Gamma_{i} \subset Y \times X \times X^{[2]}$ is of pure grade 0, we only need to show that its pull-back $\Gamma_{i}^{\prime} \subset Y \times X \times Y$ is of pure grade 0 . It is clear that $\Gamma_{i}^{\prime}$ has two irreducible components

$$
((x, y), x,(x, y)) \quad \text { and } \quad((y, x), x,(x, y)) .
$$

Then it is clear that both components are of grade 0 .

REMARK 7.2. There is a natural generically 3-to-1 morphism $X^{[2,3]} \rightarrow X^{[3]}$. With the notations of Section 5.1, this morphism is finite étale over $X^{[3]} \backslash B_{2}$, totally ramified along $B_{2} \backslash B_{3}$, and the fiber over a point of $B_{3}$ is $\mathbb{P}^{1}$. It is likely that a multiplicative Chow-Künneth decomposition for $X^{[3]}$ could also be obtained by proving an analogue of the technical descent Lemma 6.4 so that Proposition 3.7 applies to the generically finite morphism $X^{[2,3]} \rightarrow X^{[3]}$. 


\section{Acknowledgements}

We thank Kieran O'Grady and John Ottem for useful discussions concerning the map $X^{3} \rightarrow X^{[3]}$. We are grateful to the referees for comments that have helped improve the exposition. Finally, we would like to thank one of the referees and Manfred Lehn for suggesting treating the cases of the nested Hilbert schemes $X^{[1,2]}$ and $X^{[2,3]}$. The second author is supported by EPSRC Early Career Fellowship number EP/K005545/1.

\section{References}

[1] A. Beauville, 'Sur l'anneau de Chow d'une variété abélienne', Math. Ann. 273 (1986), 647-651.

[2] A. Beauville, 'On the splitting of the Bloch-Beilinson filtration', in Algebraic Cycles and Motives, Vol. 2, London Mathematical Society Lecture Notes, 344 (Cambridge University Press, 2007), 38-53.

[3] A. Beauville and C. Voisin, 'On the Chow ring of a K3 surface', J. Algebraic Geom. 13 (2004), 417-426.

[4] J. Cheah, 'Cellular decompositions for nested Hilbert schemes of points', Pacific. J. Math. 183(1) (1998), 39-90.

[5] L. Fu, Zh. Tian and Ch. Vial, 'Motivic hyperKähler resolution conjecture for generalized Kummer varieties', Preprint, 2016, arXiv:1608.04968.

[6] W. Fulton, Intersection Theory, 2nd edn, Ergebnisse der Mathematik und ihrer Grenzgebiete. 3. Folge. A Series of Modern Surveys in Mathematics, (Springer, 1998).

[7] U. Jannsen, 'Motivic sheaves and filtrations on Chow groups', in Motives (Seattle, WA, 1991), Proceedings of Symposia in Pure Mathematics, 55 (American Mathematical Society, Providence, RI, 1994), 245-302.

[8] Y. Manin, 'Correspondences, motifs and monoidal transformations', Mat. Sb. (N.S.) 77 (1968), 475-507.

[9] J. Murre, 'On the motive of an algebraic surface', J. Reine Angew. Math. 409 (1990), 190-204.

[10] J. Murre, 'On a conjectural filtration on the Chow groups of an algebraic variety. I. The general conjectures and some examples', Indag. Math. (N.S.) 4(2) (1993), 177-188.

[11] U. Riess, 'On the Chow ring of birational irreducible symplectic varieties', Manuscripta Math. 145(3-4) (2014), 473-501.

[12] M. Shen and Ch. Vial, 'The Fourier transform for certain hyperKähler fourfolds', Mem. Amer. Math. Soc. 240(1139) (2016), vii+163 pp.

[13] Ch. Vial, 'Algebraic cycles and fibrations', Doc. Math. 18 (2013), 1521-1553.

[14] Ch. Vial, 'On the motive of some hyperKähler varieties', J. Reine Angew. Math. to appear, doi:10.1515/crelle-2015-0008.

[15] C. Voisin, 'Chow rings and decomposition theorems for families of K3 surfaces and CalabiYau hypersurfaces', Geom. Topol. 16 (2012), 433-473.

[16] C. Voisin, 'The generalized Hodge and Bloch conjectures are equivalent for general complete intersections', Ann. Sci. Éc. Norm. Supér. (3) 46 (2013), 449-475. 